\title{
Adaptive fuzzy observer and robust controller for a 2-DOF robot arm
}

\section{Sangeetha Bindiganavile Nagesh}

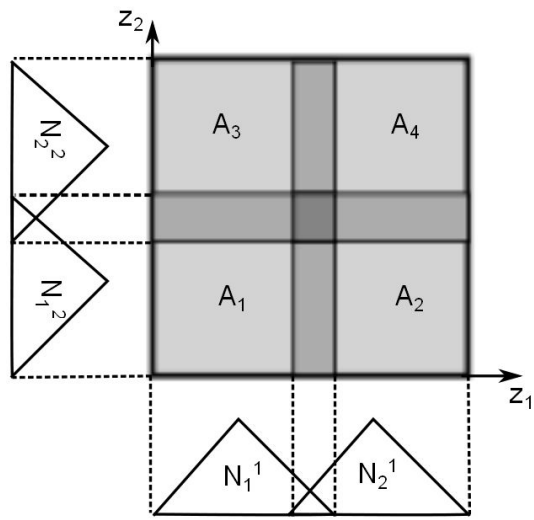

TS Fuzzy Model of the Nonlinear System

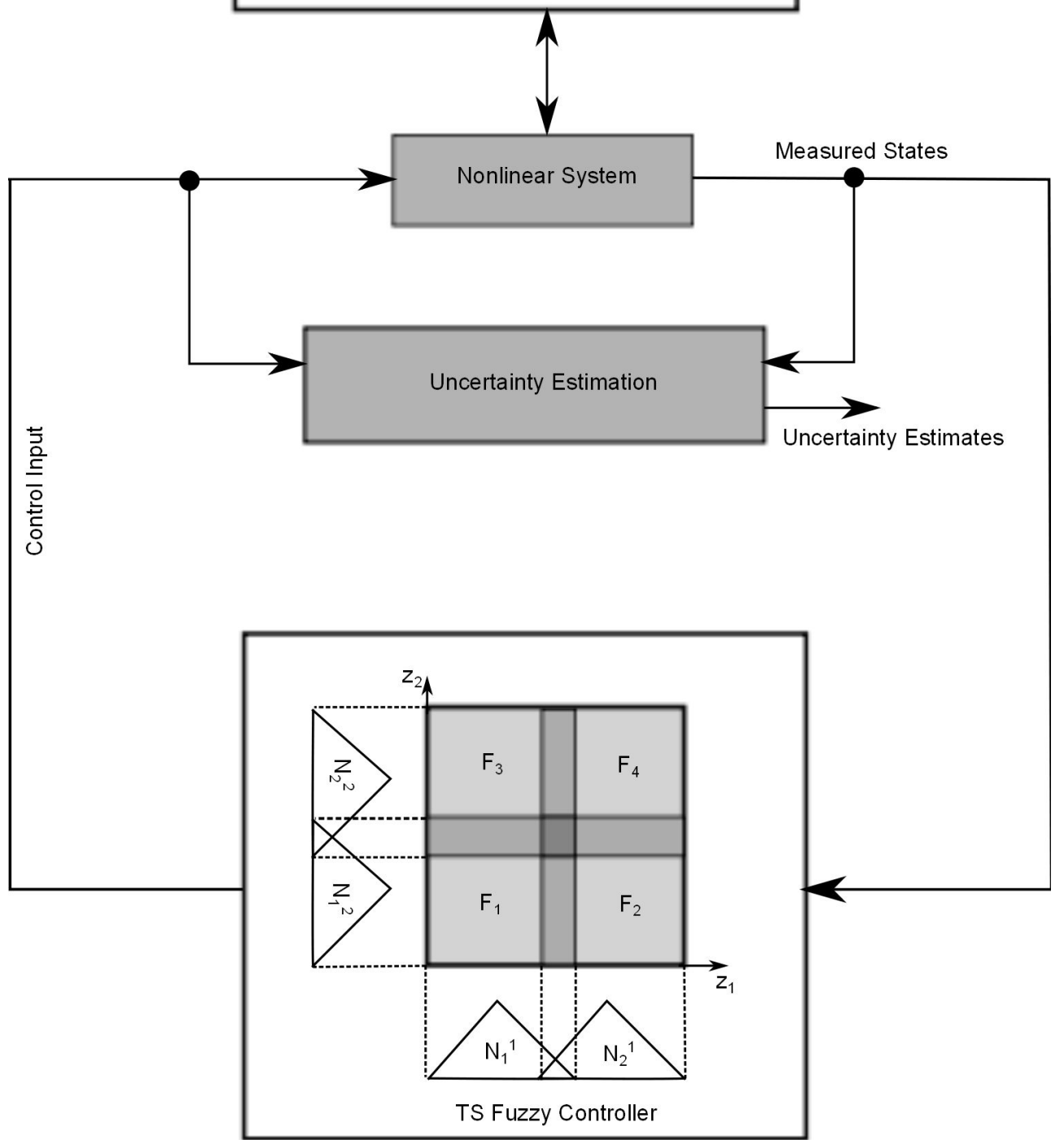





\title{
Adaptive fuzzy observer and robust controller for a 2-DOF robot arm
}

\author{
Master of Science Thesis
}

For the degree of Master of Science in Embedded Control Systems at Delft University of Technology

Sangeetha Bindiganavile Nagesh

November 10, 2011 

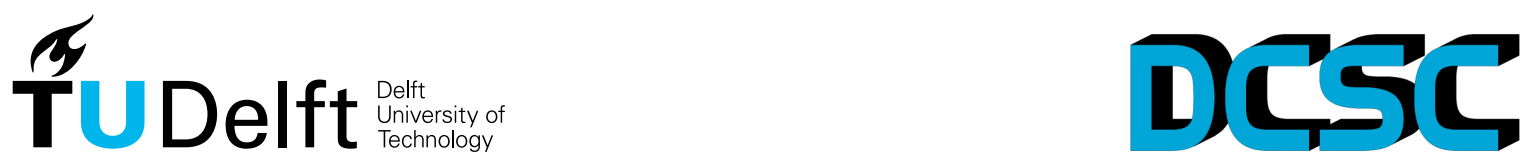

Copyright (c) Delft Center for Systems and Control (DCSC) All rights reserved. 


\section{Abstract}

Adaptive fuzzy observers have been introduced in the recent past, which are capable of estimating uncertainties along with the states of a nonlinear system represented by an uncertain Takagi-Sugeno (TS) model. Application of such an observer to obtain estimates of the uncertainties in the state matrices and subsequently use them in the control of TS fuzzy models is the subject matter of this thesis.

To demonstrate the adaptive observer and the controller design we use a 2-DOF robot arm model. The parameters of the robot arm are estimated for a laboratory-scale setup. The nonlinear model of the robot arm, consisting of six nonlinearities is simplified to contain only two nonlinearities. Then a four-rule TS fuzzy model is constructed using sector nonlinearity approach. The simplified nonlinear model and hence the fuzzy model almost exactly represent the complete nonlinear model. The mismatch in the plant and the nonlinear model is attributed to unmodelled dynamics in the state matrices. Assuming constant uncertainties in specific locations of the state matrices, an adaptive observer is used to estimate them and the simulation results are presented. The possibility to use the information about the structure of the uncertainties in the TS fuzzy model in designing the uncertainty estimation experiment is also presented. The uncertainty estimates provided by the adaptive observer are used to update the fuzzy model of the nonlinear system. The new model is used in the design of a robust state feedback stabilizing controller. Since the estimates obtained from the adaptive observer are used in controller design, the uncertainty distribution structure used in the design of both adaptive observer and the robust controller need to be same. Hence, a robust controller design is developed that uses the same uncertainty distribution structure as the adaptive observer. From the experimental results, it is concluded that stability can be guaranteed with a higher decay rate when using the updated model in robust controller design. 


\section{Table of Contents}

Acknowledgements $\quad$ v

1 Introduction $\quad 1$

2 Fuzzy models for nonlinear systems $\quad 5$

2-1 Fuzzy systems . . . . . . . . . . . . . . . . . . . . . . . . . . . . . . 5

2-2 Fuzzy models . . . . . . . . . . . . . . . . . . . . . . . . . . . . . . . 6

2-3 TS fuzzy approach to modelling nonlinear systems . . . . . . . . . . . . . . . . . 10

2-4 Lyapunov stability analysis . . . . . . . . . . . . . . . . . . . . . . . . 11

2-4-1 Lyapunov's direct method . . . . . . . . . . . . . . . . . . . . . . . . . . 13

2-4-2 Lyapunov stability analysis for LTI systems . . . . . . . . . . . . . . . . . 13

2-4-3 Stability analysis of TS systems . . . . . . . . . . . . . . . . . . . . 14

2-5 Fuzzy regulators . . . . . . . . . . . . . . . . . . . . . . . . . . . . 14

2-6 Fuzzy observers . . . . . . . . . . . . . . . . . . . . . . . . . . . . . 15

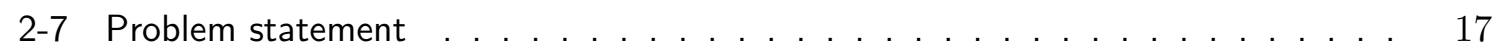

3 Fuzzy modelling of 2-DOF robot arm 21

3-1 Nonlinear model of a 2-DOF robot arm . . . . . . . . . . . . . . . . . . . . 21

3-2 Modelling of the robot arm joints . . . . . . . . . . . . . . . . . . . . . . . 22

3-3 Finding the parameters of the nonlinear 2-DOF robot arm . . . . . . . . . . . 23

3-4 Simplification of the nonlinear model . . . . . . . . . . . . . . . . . . . . 25

3-5 TS fuzzy model for the simplified 2-DOF robot arm . . . . . . . . . . . . . . . . 28

4 Robust fuzzy state feedback controller design $\quad 35$

4-1 Fuzzy state feedback controller . . . . . . . . . . . . . . . . . . . . . . . . . . 35

4-2 Uncertain TS fuzzy model . . . . . . . . . . . . . . . . . . . . . . . . . . . . . . 37

4-3 Robust stability conditions with norm-bounded uncertainty . . . . . . . . . . . . 38 
4-4 Performance specifications for the controller . . . . . . . . . . . . . . . . . . . . 43

4-4-1 Decay rate specification . . . . . . . . . . . . . . . . . . . 43

4-4-2 Constraint on control input . . . . . . . . . . . . . . . . . . . . . . 46

4-5 Controller design for 2-DOF robot arm . . . . . . . . . . . . . . . . . . . . . . 46

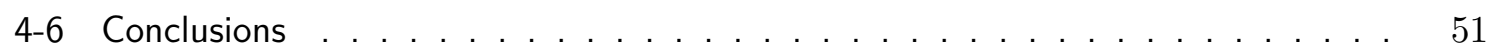

5 Adaptive observer $\quad 53$

5-1 Introduction . . . . . . . . . . . . . . . . . . . . . . . . . . . 53

5-2 Adaptive observer design for the simplified 2-DOF robot arm . . . . . . . . . . . 56

5-3 Uncertainty structure information . . . . . . . . . . . . . . . . . . . . . . 66

5-4 Conclusions .............................. . . 68

6 Adaptive observer and robust controller $\quad 69$

6-1 Uncertainty estimates in controller design . . . . . . . . . . . . . . . . . . . . . 69

6-2 Parallel operation of adaptive observer and robust controller . . . . . . . . . . . 71

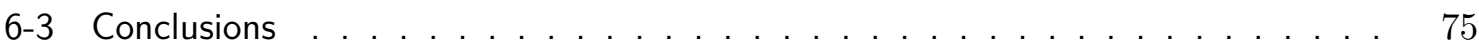

7 Conclusions and proposals for future work $\quad 77$

7-1 Conclusions . . . . . . . . . . . . . . . . . . . . . . . . . . . . . . . . . . . . . 78

7-2 Future research proposals . . . . . . . . . . . . . . . . . . . . . 80

A Sector nonlinearity approach $\quad 83$

B Application examples $\quad 87$

B-1 2-DOF helicopter model . . . . . . . . . . . . . . . . . . . . . . . 87

B-2 Delft robot $a r m$. . . . . . . . . . . . . . . . . . . . . . . . . 89

B-3 2-DOF robot $\mathrm{arm}$. . . . . . . . . . . . . . . . . . . . . 92

C Robust controller design with relaxed stability conditions 95

D Computational complexity 101

D-1 Number of LMls in robust controller design . . . . . . . . . . . . . . . . . . . . 101

D-2 Number of LMls in adaptive observer design . . . . . . . . . . . . . . . . . . . . 102

$\begin{array}{lr}\text { E Glossary } & 105\end{array}$

List of Acronyms . . . . . . . . . . . . . . . . . . . . . . . . . . . . . . . . . . . 105

List of Symbols . . . . . . . . . . . . . . . . . . . . . . . . . . . . 105

$\begin{array}{ll}\text { References } & 107\end{array}$ 


\section{Acknowledgements}

I sincerely thank my supervisor prof. dr. ir. Robert Babuška for his valuable guidance during the course of my thesis work. I thank dr. ir. Zsófia Lendek for her ever enthusiastic supervision and motivating guidance. My gratitude also goes to ir. Amol Khalate who guided me with appropriate inputs during the course of my thesis. I express my appreciation for the time, patience and understanding each one of them have shown towards me. Their timely suggestions have been of great help in leading me to the completion of my thesis.

I thank my mother and sister for their unconditional support and for being my source of strength. I thank my friends for their care and understanding.

Delft, University of Technology

Sangeetha Bindiganavile Nagesh

November 10, 2011 


\section{Chapter 1}

\section{Introduction}

Systems generally are nonlinear in nature. Traditional control strategies developed involve control methods that approximate them as linear systems. However, the associated disadvantage is that the linearized systems fail to completely represent the real plants that are highly nonlinear. Linear control theory is aimed at the analysis and control of nonlinear plants through linearization about an equilibrium point. This results in controllers being applicable only around the equilibrium point [1]. As the applications nowadays concern the area of precise control and control over wide operating ranges, control of nonlinear systems has been a subject of research.

Methods for analysis and design of general nonlinear systems have been described in [2]. A knowledge based way of representing and controlling nonlinear systems is proposed in [3]. Specifically, Takagi-Sugeno (TS) model-based control design strategies have been a popular field of research due to their ability to closely approximate or exactly represent the nonlinear systems [4]. Since then, this promising area has been widely researched.

In this thesis, nonlinear systems represented in the form of TS fuzzy models are considered. In particular, uncertain TS fuzzy models are considered to accommodate uncertainty in the system models. The TS fuzzy model is created using the sector nonlinearity approach [5]. In this approach, the nonlinearities in the system are termed as scheduling variables and fuzzy membership functions are associated with them. The TS fuzzy representation of the nonlinear system contains a number of rules with each rule associated with a linear state space model. The value of the scheduling variables decide to what extent these linear models are valid. The fuzzy combination of the rules exactly represents the nonlinear system. An introduction to sector nonlinearity and its application to three examples are presented in Appendices A and B.

Control of nonlinear systems represented by TS fuzzy models has been a topic of research since the inception of the idea in 1985 [3]. One of the widely researched controller structure is Parallel Distributed Compensator (PDC) $[6,7]$ in which each controller rule is designed from the corresponding rule of the TS model. The controller rules have linear state feedback control laws associated with them. Considering uncertain TS fuzzy models as the target 
area of application, design of state feedback controllers that accommodate for a particular representation of norm bounded uncertainty blocks in the state and the input matrices of the linear state space models are available $[5,8]$. This design area can be categorized as TS fuzzy robust state feedback controller design for norm bounded uncertainties. Performance indices like the rate of decay of the states and input constraints can be added to the controller design $[5]$.

In practice, all the states of a system may not be measurable due to factors such as cost and sensor availability. In such a situation, observers are used in the design to provide estimates of unmeasured states. In the recent past, observer design that can estimate unmodelled dynamics along with the states of a system represented by a TS fuzzy model has been presented [1]. These observers, referred to in the literature as adaptive observers, are shown to be asymptotically stable. They are shown to be useful to estimate constant or slowly varying uncertainties in the local linear models of the TS system.

In this thesis, we use the adaptive observer to estimate a constant uncertainty in the state matrices of uncertain TS fuzzy models. The influence of thus obtained uncertainty estimates on the controller design is analyzed. This analysis necessitates the uncertainty considered in the designs of both robust state feedback controller and the adaptive observer to be represented by same uncertainty distribution structure. Hence a robust state feedback controller design is developed for the uncertainty distribution structure similar to that used in adaptive observer in the literature. The influence of a controller stabilizing the system, while the adaptive observer is estimating the uncertainties is also investigated. Further, the possibility to design uncertainty estimation experiments based on the structure of the TS fuzzy models is investigated. A 2-DOF robot arm is chosen as an application to illustrate the robust controller design, the adaptive observer and to analyze the effects of using an updated model in the robust controller design.

In the following, the layout of chapters in this thesis is outlined. Chapter 2 presents the general concepts of fuzzy systems and describes in detail TS fuzzy systems. The chapter also presents stability analysis of TS fuzzy systems, and a brief introduction to TS fuzzy regulators and TS fuzzy observers. The final part of this chapter contains the mathematical details of the research problem addressed in this thesis. Chapter 3 describes fuzzy modelling of the 2-DOF robot arm. The TS fuzzy model of the 2-DOF robot arm is used in the rest of the chapters.

Chapter 4 presents the robust state feedback controller design for the uncertainty distribution structure similar to that used in adaptive observers [1]. In the first part of the chapter, stabilization criteria for systems controlled by state feedback controllers is presented. This is followed by the mathematical formulations of the robust state feedback controller. Performance criteria namely the decay rate and control input constraint are considered as part of the controller design. This is followed by simulation results for the simplified 2-DOF robot arm model assuming a constant uncertainty in the state matrices.

Chapter 5 presents the design of adaptive observer for a constant uncertainty in the state matrices of the 2-DOF robot arm model. An introduction to the adaptive observer design concept is presented in the first part of the chapter followed by design of the adaptive observer for the robot arm and simulation results. The effect of increasing the uncertainty norm bound for which the adaptive observer is designed, is illustrated through experimental results. A 
possibility to exploit the structure of a TS fuzzy model in designing the estimation experiments is provided for the specific example of simplified 2-DOF robot arm.

Chapter 6 presents the use of the uncertainty estimates obtained from the adaptive observer in redesigning the robust state feedback controller with a lower uncertainty norm bound and a higher decay rate for the 2-DOF robot arm. The possibility of using the adaptive observer and robust state feedback controller in parallel operation is discussed, assuming a constant uncertainty in the state matrices of the local linear models of a TS fuzzy system.

Chapter 7 presents the conclusions derived from the work done, followed by future work proposals. 


\section{Chapter 2}

\section{Fuzzy models for nonlinear systems}

In this chapter, a brief overview of fuzzy systems is presented and Takagi-Sugeno (TS) fuzzy systems are discussed in particular. Stability analysis of TS fuzzy models based on Lyapunov stability theory is presented subsequently. A brief introduction to TS regulators and observers is provided. Finally, in the last section, the mathematical details of the research problem addressed in this thesis is presented.

\section{2-1 Fuzzy systems}

Systems that make use of if-then type of rules to characterize the system behaviour are called fuzzy systems. The if-then rules are usually formulated based on expert practical knowledge about the system. The following introduces the different attributes of fuzzy systems and illustrate them on examples.

Fuzzy logic is an analytical way of representing the human way of thought process and imprecise, inexact phenomena. For instance, this approach makes it possible to mathematically represent a statement like:

\section{"John is tall"}

It is to be noted in the statement above that the word tall is an inexact measure of height and is relative. Fuzzy logic enables the representation of this inexact measure as a degree of tallness. Statements like John is tall are called fuzzy propositions. Unlike in classical logic where an element either belongs completely $(100 \%)$ to a particular set or not $(0 \%)$, in fuzzy sets an element can belong to a particular set with a certain degree. This certain degree of belonging to a particular set is indicated by the membership value of the element in the set. Since the degree of membership can range between $0 \%$ and $100 \%$, the membership value of an element in a set is always in the closed interval [0.0, 1.0].

A relation that provides for every element of the set a particular membership value is called a membership function. A set where every element associates itself with the set with a 
membership value ranging between 0.0 and 1.0 is called a fuzzy set. Let us consider for example, the following statement:

"The Speed is low"

The membership function of the fuzzy variable Speed can be represented as in figure 2-1. In

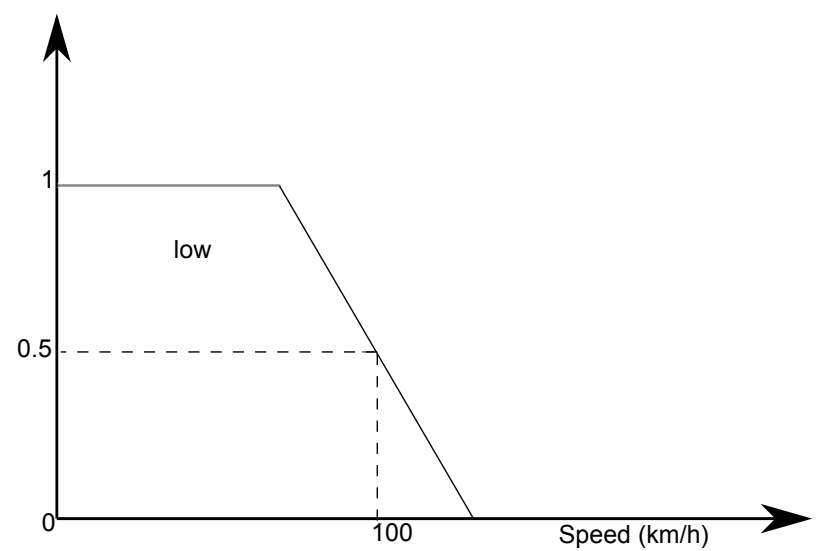

Figure 2-1: Membership function for the fuzzy variable Speed.

the above statement,

- Speed is a fuzzy variable which can take the value low (a fuzzy set).

- A crisp value of the real physical quantity speed, like $100 \mathrm{~km} / \mathrm{h}$, can have a degree of membership 0.5 in the fuzzy set low, that is, $\mu_{l o w}(100 \mathrm{~km} / \mathrm{h})=0.5$.

Hence if $X$ is the universe of discourse, $A$ is a fuzzy set with every member of $A$ associated with a membership value and $x$ a crisp value in $X$, we can represent the relation as:

$$
\mu_{A}(x): X \rightarrow[0,1]
$$

Most commonly used membership functions are triangular and trapezoidal [9].

\section{2-2 Fuzzy models}

Traditional methods for process/plant modelling involve formulation of mathematical equations describing the system dynamics. However, a mathematical formulation is not suitable in some situations for instance, complex chemical processes which involve interaction of several physical laws. In such a scenario, availability of human experience and knowledge about a process/plant in the form of if-then (more formally, antecedent-consequent) rules to formulate process/plant models is considered to be a promising direction. The models that evolve on this paradigm came to be known as fuzzy models.

There are two major classes of fuzzy models called Mamdani fuzzy models and TS fuzzy models. 
In Mamdani models, the antecedents and the consequents are both fuzzy propositions. For instance, a Mamdani fuzzy model of a system with $r$ rules is as shown below:

$$
R_{i}: \text { If } x \text { is } A_{i} \text { then } y \text { is } B_{i}, \quad i=1,2, \ldots, r
$$

where

- " $x$ is $A_{i}$ " corresponds to the antecedent proposition of the rule.

- " $y$ is $B_{i}$ " corresponds to the consequent proposition of the rule.

- $x$ is the input fuzzy/linguistic variable.

- $A_{i}$ is the antecedent fuzzy/linguistic term which is associated with a membership function.

- $y$ is the output fuzzy/linguistic variable.

- $B_{i}$ is the consequent fuzzy/linguistic term which is associated with a membership function.

- $r$ is the number of rules.

In TS models, the antecedents are fuzzy sets and the consequents are crisp functions of the antecedent variables. A TS model of a system with $r$ rules is for instance:

$$
R_{i}: \text { If } x \text { is } A_{i} \text { then } y_{i}=f_{i}(x), \quad i=1,2, \ldots, r
$$

where $f_{i}(x)$ is a function of the antecedent variables and all the other variables in (2-3) are as described above.

Fuzzy models can be used to represent processes or to develop controllers. For more information on the different kinds of fuzzy models and further background material, the reader is referred to $[10]$ and the references therein.

A typical fuzzy system can be represented as shown in Figure 2-2.

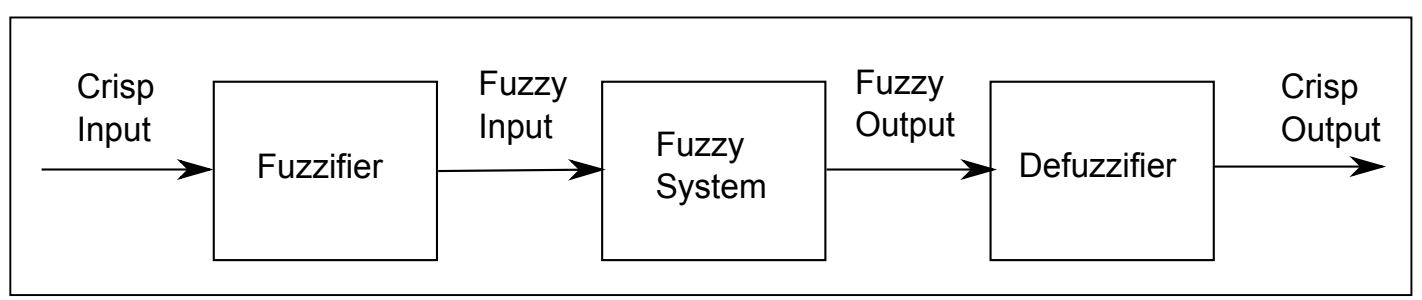

Figure 2-2: A typical fuzzy system.

The components of Figure 2-2 and their functions is described below.

- Fuzzifier/Fuzzification: The process of mapping a crisp input to a membership value and hence to a fuzzy value is called fuzzification. 
- Inference: The result of fuzzification is a fuzzy value which is then passed through the fuzzy system itself or the fuzzy inference engine. The fuzzy inference engine consists of a set of rules. For instance, consider the rule

\section{"If speed is low, then fuel injected is high"}

The above statement indicates that when speed is low, an action is to be performed to inject more fuel into the engine thus increasing the speed.

- Defuzzifier/Defuzzification: The fuzzy inference engine results in a fuzzy output. In the example above, it says the result is high fuel injection. The output value does not indicate what the exact quantity of fuel to be injected is. The task of providing an exact value is accomplished by the defuzzifier which performs a fuzzy value to crisp value conversion. This gives the exact amount of fuel to be injected. The process is called defuzzification.

In many fuzzy control systems there are several inputs that are fuzzified and passed onto the inference engine. The inference engine usually consists of more than one rule to decide on the fuzzy output value. The defuzzification process involves a one-to-one conversion between a fuzzy value to a crisp value. There are certain concepts central to the idea of inference engine which are briefly presented below.

Since a fuzzy system can have many inputs with each input fuzzified by a fuzzifier, the inference engine needs to combine all the fuzzified inputs as per the rule base to produce a fuzzified output. This process is called inference and makes use of fuzzy logical operators to combine the fuzzified inputs. The fuzzy rules that we described earlier are the decision makers in the inference process. An example of a fuzzy rule is shown below: Consider the rule,

$$
\text { If } x \text { is } A \text { AND } y \text { is } B \text {, then } z \text { is } C
$$

where

- $x, y$ and $z$ are fuzzy variables.

- $A, B$ and $C$ are fuzzy sets.

The membership values of the variables $x$ and $y$ in the fuzzy sets $A$ and $B$ can be combined to obtain a degree of relevance of the entire rule. To perform this kind of combination, three basic operations on fuzzy sets are defined: complement of a fuzzy set, intersection of fuzzy sets and union of fuzzy sets.

Definition 1. [11] Complement of a fuzzy set: If $A$ is a fuzzy set defined in the universe of discourse $X$, the complement of the fuzzy set $A$ is defined as:

$$
A^{\prime}=\left\{x \in X \mid \mu_{A^{\prime}}(x)=1-\mu_{A}(x)\right\}
$$

Definition 2. [11] Intersection of fuzzy sets: If $A$ and $B$ are two fuzzy sets defined in $X$, the intersection of the two sets $A$ and $B$ is defined as:

$$
C=A \cap B
$$

with

$$
\mu_{C}(x)=\min \left(\mu_{A}(x), \mu_{B}(x)\right)
$$


Definition 3. [11] Union of fuzzy sets: If $A$ and $B$ are two fuzzy sets defined in $X$, the union of the sets $A$ and $B$ is defined as:

$$
C=A \cup B
$$

with

$$
\mu_{C}(x)=\max \left(\mu_{A}(x), \mu_{B}(x)\right)
$$

Other definitions of complement also exist (e.g: $\lambda$ complement [12]). Similarly, other intersection and union operators exist [11]. A commonly used intersection operator is the product operator since it results in smooth operations. The product operator is as defined below:

$$
\mu_{C}(x)=\mu_{A}(x) \mu_{B}(x)
$$

An algebraic sum based union operator is for instance:

$$
\mu_{C}(x)=\mu_{A}(x)+\mu_{B}(x)-\mu_{A}(x) \mu_{B}(x)
$$

The inference mechanism used may differ based on the kind of fuzzy model used. Hence, Mamdani and TS inference mechanisms are possible. For more information on these mechanisms, the interested reader is referred to [13] and the MATLAB Fuzzy Logic Toolbox.

The number of rules in a rule base is generally more than one. The effects of all the rules have to be combined to get an output which is representative of the entire rule base. This is called aggregation. The last component of the system is the defuzzifier which converts the fuzzy output value after aggregation to a crisp value that can be used to control a plant.

The concept of aggregation/defuzzification is illustrated on an example of TS fuzzy model in which the output membership function is linear in the input variable. Consider the rule base,

$$
\begin{aligned}
& R_{1}: \text { If } x \text { is } A_{1} \text {, then } y=5 x \\
& R_{2}: \text { If } x \text { is } A_{2}, \text { then } y=3 x
\end{aligned}
$$

The membership functions for the sets $A_{1}$ and $A_{2}$ are taken as in the Figure 2-3. Given the

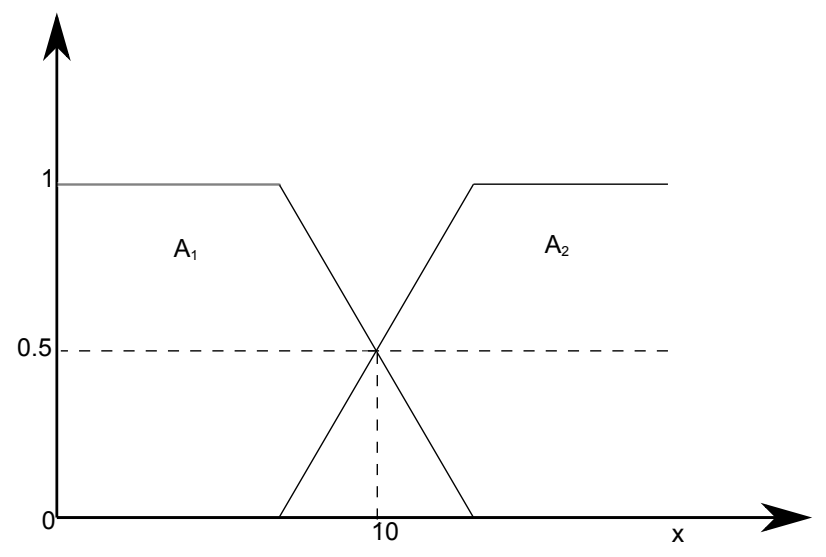

Figure 2-3: Membership value of variable $x$ in the sets $A_{1}$ and $A_{2}$.

input $x=10$, the membership values of $x=10$ in the sets $A_{1}$ and $A_{2}$ can be found from 
the membership functions Figure 2-3 as $\mu_{A_{1}}(x)=0.5$ and $\mu_{A_{2}}(x)=0.5$. The output of the system is

$$
y=\frac{\mu_{A_{1}}(x) y_{R_{1}}+\mu_{A_{2}}(x) y_{R_{2}}}{\mu_{A_{1}}(x)+\mu_{A_{2}}(x)}
$$

where $y_{R_{1}}$ and $y_{R_{2}}$ are the outputs of rule 1 and rule 2 , respectively.

In case of Mamdani models, the aggregration and defuzzification processes are more distinct from one another than in TS models. The interested reader is referred to [11].

Further material in this report will focus only on the TS fuzzy model as this thesis involves investigation of properties of nonlinear systems represented using TS fuzzy models. Information pertaining to the use of Mamdani fuzzy models for nonlinear systems is not discussed as it is out of the scope of this master thesis.

TS models have been proven to be universal approximators [4]. Thus TS models can be used to represent any nonlinear system to a desired degree of accuracy $[3,14]$. The next section will briefly describe the TS fuzzy approach of modelling nonlinear systems.

\section{2-3 TS fuzzy approach to modelling nonlinear systems}

Dynamic TS models represent a nonlinear plant by a combination of several locally valid linear models. Each linear model is valid in the regions of state space as indicated by the antecedents of the fuzzy rule. A fuzzy combination of several such rules provides a close approximation or an exact representation of the nonlinear plant.

The TS models used to represent the nonlinear system can be either continuous time or discrete time. In this thesis, continuous time TS systems are considered.

A TS model for a nonlinear system is generally of the form:

$$
\begin{aligned}
& R_{i} \text { : If } z_{1} \text { is } N_{i}^{1} \text { and } z_{2} \text { is } N_{i}^{2}, \ldots \text { and } z_{p} \text { is } N_{i}^{p} \text {, then } \\
& \qquad \begin{aligned}
\dot{\boldsymbol{x}} & =A_{i} \boldsymbol{x}+B_{i} \boldsymbol{u} \\
\boldsymbol{y} & =C_{i} \boldsymbol{x}
\end{aligned}
\end{aligned}
$$

for $i=1,2, \ldots, r$

- The variables $z_{1}, z_{2}, \ldots, z_{p}$ are the antecedent or the scheduling variables that are dependent on states, inputs or other external factors.

- $N_{i}^{1}, N_{i}^{2} \ldots$ and $N_{i}^{p}$ are the fuzzy sets of the $i^{t h}$ rule.

- $\boldsymbol{x}$ represents the state vector and $\boldsymbol{u}$ represents the input vector.

- $A_{i}$ and $B_{i}$ represent the state and the input matrix of the $i^{\text {th }}$ model or subsystem. 
Let $w_{i j}\left(z_{j}\right)$ represent the membership value of $z_{j}$ in fuzzy set $N_{i}^{j}$ in rule $i$. Then the degree of relevance of the entire antecedent in equation (2-13) can be represented as $w_{i}(\boldsymbol{z})$, where $\boldsymbol{z}=\left[z_{1}, z_{2}, \ldots, z_{p}\right]^{T}$ is the scheduling vector comprising all the scheduling variables.

If there are $r$ rules to represent the system, then we can represent the entire nonlinear system by the following fuzzy model :

$$
\dot{\boldsymbol{x}}=\frac{\sum_{i=1}^{r} w_{i}(\boldsymbol{z})\left(A_{i} \boldsymbol{x}+B_{i} \boldsymbol{u}\right)}{\sum_{i=1}^{r} w_{i}(\boldsymbol{z})}
$$

Let

$$
h_{i}(\boldsymbol{z})=\frac{w_{i}(\boldsymbol{z})}{\sum_{i=1}^{r} w_{i}(\boldsymbol{z})}
$$

be the normalized value of the degree of relevance of the $i^{t h}$ rule.

Hence, the system in (2-13) can be represented as

$$
\begin{aligned}
\dot{\boldsymbol{x}} & =\sum_{i=1}^{r} h_{i}(\boldsymbol{z})\left(A_{i} \boldsymbol{x}+B_{i} \boldsymbol{u}\right) \\
\boldsymbol{y} & =\sum_{i=1}^{r} h_{i}(\boldsymbol{z})\left(C_{i} \boldsymbol{x}\right)
\end{aligned}
$$

TS fuzzy models with a bias in the consequent parts are called affine TS fuzzy models and the biases are called affine terms. These systems are represented as

$$
\begin{aligned}
& R_{i}: \text { If } z_{1} \text { is } N_{i}^{1} \text { and } z_{2} \text { is } N_{i}^{2}, \ldots \text { and } z_{p} \text { is } N_{i}^{p} \text {, then } \\
& \qquad \begin{aligned}
\dot{\boldsymbol{x}} & =A_{i} \boldsymbol{x}+B_{i} \boldsymbol{u}+a_{i} \\
\boldsymbol{y} & =C_{i} \boldsymbol{x}+c_{i}
\end{aligned}
\end{aligned}
$$

for $i=1,2, \ldots, r$, where $a_{i}$ and $c_{i}$ are the biases in the local models and the remaining terms are as defined for (2-13). Representation similar to (2-14) of the nonlinear systems with affine terms can be formulated, but are not detailed here.

In the following section, stability analysis concepts are introduced. Lyapunov stability analysis for linear systems and its extension for TS fuzzy models is presented.

\section{2-4 Lyapunov stability analysis}

Stability of a control system is of crucial importance since any functionality that is desired is performed well only if the system is stable. The Lyapunov method is the most generally used method to perform stability analysis of nonlinear systems. Some important definitions are presented before continuing to the Lyapunov stability method. 
Consider a system in state space form

$$
\dot{\boldsymbol{x}}=f(\boldsymbol{x}, t)
$$

where

- $\boldsymbol{x} \in \mathbb{R}^{n_{x}}$ is the n-dimensional state vector.

- $f(\boldsymbol{x}, t) \in \mathbb{R}^{n_{x}}$ is a n-dimensional vector consisting of functions of elements of the state vector $\boldsymbol{x}$ and time $t$.

If the initial conditions are $\boldsymbol{x}=\boldsymbol{x}_{0}$ at $t=t_{0}$, then the solution of the system is $\phi\left(t, \boldsymbol{x}_{0}, t_{0}\right)$.

Definition 4. [15] Equilibrium state: is a state of the system where the variation in the states become 0 (that is, the derivatives of the states are zero).

$$
f\left(\boldsymbol{x}_{e}, t\right)=0 \quad \forall t
$$

A nonlinear system can have more than one equilibrium state. Any equilibrium state can be analyzed by considering it to be situated at the origin of the state space. This is because an equilibrium state can be shifted to the origin by coordinate translation.

Definition 5. [15] Lyapunov stability: An equilibrium state $\boldsymbol{x}_{e}$ is stable in the sense of Lyapunov, if there exists $\delta$ and $\epsilon$ such that starting from an initial state of $\boldsymbol{x}_{0}$ within a radius $\delta$ from the equilibrium point, the state of the system remains within a radius $\epsilon$ from the equilibrium as $t \rightarrow \infty$.

$$
\text { If }\left\|\boldsymbol{x}_{0}-\boldsymbol{x}_{e}\right\| \leq \delta, \text { then }\left\|\phi\left(t, \boldsymbol{x}_{0}, t_{0}\right)-\boldsymbol{x}_{e}\right\| \leq \epsilon \quad \forall t \geq t_{0}
$$

$\delta$ is usually considered as a fraction of $\epsilon$ and $\delta$ may be dependent on $t_{0}$. If $\delta$ is not dependent on $t_{0}$, then the equilibrium is uniformly stable.

Definition 6. [15] Asymptotic stability: An equilibrium state $\boldsymbol{x}_{e}$ is asymptotically stable if starting from an initial state $\boldsymbol{x}_{0}$ within a radius $\delta$ from the equilibrium, the state of the system converges to the equilibrium.

Definition 7. [15] Asymptotic stability in the large: If asymptotic stability holds for all states from any initial condition, then the equilibrium is considered to be asymptotically stable in the large.

Definition 8. [2] Exponential stability and decay rate: The equilibrium point $\boldsymbol{x}_{e}=0$ is exponentially stable if there exist positive constants $\delta, k$ and $\alpha$ such that

$$
\|\boldsymbol{x}(t)\| \leq k\left\|\boldsymbol{x}_{0}\right\| e^{-\alpha\left(t-t_{0}\right)}, \quad \forall\left\|\boldsymbol{x}_{0}\right\|<\delta \text { and } \forall t \geq t_{0}
$$

and globally exponentially stable if the above condition is satisfied for any initial state $\boldsymbol{x}_{0}$. The largest constant $\alpha$ that can be utilized in (2-21) is called the decay rate. 


\section{2-4-1 Lyapunov's direct method}

This method of determining stability is based on the idea that for a system with an asymptotically stable equilibrium state, the energy of the system displaced from its equilibrium decreases with time and assumes a minimum value at equilibrium state. It makes use of a Lyapunov function similar to energy functions to determine stability. The Lyapunov function is denoted by $V(\boldsymbol{x})$ where $\boldsymbol{x}$ is the state vector of the system. Lyapunov's stability theorem is as follows.

Theorem 1. Consider a system described by,

$$
\dot{\boldsymbol{x}}=f(\boldsymbol{x}, t) \text { where } \dot{\boldsymbol{x}}=0 \text { at } \boldsymbol{x}=0 \text {. }
$$

If there exists a scalar function $V(\boldsymbol{x}, t)$ having continuous partial derivatives and satisfying the conditions:

- $V(\boldsymbol{x}, t)$ is positive definite and

- $\dot{V}(\boldsymbol{x}, t)$ is negative definite

then the equilibrium state at origin is uniformly asymptotically stable in the large.

The condition that $\dot{V}(\boldsymbol{x}, t)$ is negative definite can be relaxed to $\dot{V}(\boldsymbol{x}, t)$ is negative semidefinite, in which case the condition that $\dot{V}(\boldsymbol{x}, t)$ does not vanish along any trajectory except at origin is imposed.

The following subsection provides the mathematical details pertaining to Lyapunov stability analysis of linear time invariant (LTI) system which will be used later.

\section{2-4-2 Lyapunov stability analysis for LTI systems}

Consider an autonomous LTI system represented as

$$
\dot{\boldsymbol{x}}=A \boldsymbol{x}
$$

where $A \in \mathbb{R}^{n_{x} \times n_{x}}$ is the state matrix whose size corresponds to the dimensions of the state vector. The system (2-23) is obtained by linearizing the nonlinear system (2-22). A possible Lyapunov function for the system $(2-23)$ is:

$$
V(\boldsymbol{x})=\boldsymbol{x}^{T} P \boldsymbol{x}
$$

and hence,

$$
\begin{aligned}
\dot{V}(\boldsymbol{x}) & =\dot{\boldsymbol{x}}^{T} P \boldsymbol{x}+\boldsymbol{x}^{T} P \dot{\boldsymbol{x}} \\
& =(A \boldsymbol{x})^{T} P \boldsymbol{x}+\boldsymbol{x}^{T} P(A \boldsymbol{x}) \\
& =\boldsymbol{x}^{T}\left(A^{T} P+P A\right) \boldsymbol{x}
\end{aligned}
$$

For asymptotic stability, since we require $\dot{V}(\boldsymbol{x})$ to be negative definite, we can consider

$$
\dot{V}(\boldsymbol{x})=-\boldsymbol{x}^{T} Q \boldsymbol{x}<0
$$


with $Q=-\left(A^{T} P+P A\right)$ to be positive definite. The Lyapunov function (2-24) is called quadratic Lyapunov function and the system (2-23) is called quadratically stable if the condition (2-26) is true.

The next section presents the Lyapunov stability analysis for continuous time TS fuzzy systems.

\section{2-4-3 Stability analysis of TS systems}

For linear systems of the form,

$$
\dot{\boldsymbol{x}}=A \boldsymbol{x}
$$

stability is confirmed by the existence of a positive definite matrix $P$ satisfying the inequality

$$
A^{T} P+P A<0
$$

which ensures that there exists a Lyapunov function $V(\boldsymbol{x})$ resulting in $\dot{V}(\boldsymbol{x})<0$ (See Section 2-4-2 for details).

Consider the system represented by equation (2-13). Every local model of the TS system is individually stable if a separate positive definite matrix $P$ exists for every $A_{i}$ satisfying the this equation $A_{i}^{T} P+P A_{i}<0$. However, it has been proven in [16] that a nonlinear system represented by a fuzzy system need not be globally stable even if all the component linear systems $\left(A_{i}\right.$ matrices) are stable.

Based on Lyapunov stability theory, the conditions for global asymptotic stability of a TS fuzzy model are presented in [17]. The conditions are as per the following theorem.

Theorem 2. [1']] If there exists a common symmetric positive definite matrix $P \in \mathbb{R}^{n \times n}$ satisfying

$$
A_{i}^{T} P+P A_{i}<0, \quad i=1,2, \ldots r
$$

then the TS fuzzy model in equation (2-13) is globally asymptotically stable.

In the following two sections, a brief introduction is given to TS fuzzy regulators and observers thus providing the background concepts required to present the mathematical details of the problem addressed in this thesis in the last section of this chapter.

\section{2-5 Fuzzy regulators}

Fuzzy regulators have been introduced in [6] and [7] to stabilize fuzzy systems. Regulators are in general designed to ensure that the states decay to 0 as time increases infinitely $(\boldsymbol{x}(t) \rightarrow 0$ as $t \rightarrow \infty)$. The most common technique used is Parallel Distributed Compensator (PDC) for TS fuzzy systems. In this technique every rule of the fuzzy system will be compensated by a corresponding rule of the regulator with a linear feedback law as its consequent.

Let us consider the system

$$
R_{i}: \text { If } z_{1} \text { is } N_{i}^{1} \text { and } z_{2} \text { is } N_{i}^{2}, \ldots \text { and } z_{p} \text { is } N_{i}^{p} \text {, then }
$$




$$
\begin{aligned}
\dot{\boldsymbol{x}} & =A_{i} \boldsymbol{x}+B_{i} \boldsymbol{u} \\
\boldsymbol{y} & =C_{i} \boldsymbol{x}
\end{aligned}
$$

for $i=1,2, \ldots, r$.

The rule of the fuzzy regulator corresponding to the system (2-30) can be written as:

$$
R_{i} \text { : If } z_{1} \text { is } N_{i}^{1} \text { and } z_{2} \text { is } N_{i}^{2} \ldots \text { and } z_{p} \text { is } N_{i}^{p} \text {, then } \boldsymbol{u}=-F_{i} \boldsymbol{x}
$$

for $i=1,2, \ldots, r$ where

- $R_{i}$ represents the $i^{\text {th }}$ rule of the controller.

- $F_{i}$ represents the gain matrix of the linear state feedback corresponding to the $i^{\text {th }}$ rule.

Considering $\boldsymbol{u}=-\sum_{i=1}^{r} h_{i}(\boldsymbol{z}) F_{i} \boldsymbol{x}$ in the equation of the system (2-16) we have,

$$
\dot{\boldsymbol{x}}=\sum_{i=1}^{r} \sum_{j=1}^{r} h_{i}(\boldsymbol{z}) h_{j}(\boldsymbol{z})\left(A_{i}-B_{i} F_{j}\right) \boldsymbol{x}
$$

PDC controllers for TS fuzzy models have been designed in [18] and is one of the first papers where the linear matrix inequality (LMI) technique is used to prove asymptotic stability. The paper also referred to the dependency of the basin of attraction of an equilibrium state on membership functions.

Results dealing with stabilization of TS fuzzy models by PDCs are presented in [7, 19]. Performance specifications such as decay rate, control input constraint and output constraints are considered in [19]. Given that the initial conditions $\boldsymbol{x}(0)$ are known such that $\|\boldsymbol{x}(0)\| \leq \phi$, LMI conditions for $\|\boldsymbol{u}(t)\| \leq \zeta$ to be true for all $t \geq 0$ are given in [19]. Here $\zeta$ is the upper bound on the control input norm and $\phi$ is the bound on initial condition norm. Further details on these results are discussed in Chapter 4 that presents the design of robust state feedback controller.

Fuzzy regulators can be used to control the TS fuzzy systems provided that the states of the system are measured. However, due to practical constraints, sensors may not be available to measure all the states. Then observers can be used to provide an estimate of the states of the system. The following section presents the concept of TS fuzzy observers.

\section{2-6 Fuzzy observers}

Observers use the control input and the system's output to estimate the system's states. They are designed to ensure that the estimated states converge to the true states as time progresses infinitely $((\boldsymbol{x}(t)-\widehat{\boldsymbol{x}}(t)) \rightarrow 0$ as $t \rightarrow \infty)$.

An approach for designing fuzzy observers has been given in $[6,7]$, the outline of which is given in the following. Consider the TS system

$$
R_{i}: \text { If } z_{1} \text { is } N_{i}^{1} \text { and } z_{2} \text { is } N_{i}^{2} \ldots \text { and } z_{p} \text { is } N_{i}^{p} \text {, then }
$$




$$
\begin{aligned}
\dot{\boldsymbol{x}} & =A_{i} \boldsymbol{x}+B_{i} \boldsymbol{u} \\
\boldsymbol{y} & =C_{i} \boldsymbol{x}
\end{aligned}
$$

for $i=1,2, \ldots, r$.

The observer for the fuzzy system in (2-33) can be represented as:

$$
\begin{aligned}
& R_{i}: \text { If } z_{1} \text { is } N_{i}^{1} \text { and } z_{2} \text { is } N_{i}^{2} \ldots \text { and } z_{p} \text { is } N_{i}^{p} \text {, then } \\
& \qquad \begin{aligned}
\dot{\widehat{\boldsymbol{x}}} & =A_{i} \widehat{\boldsymbol{x}}+B_{i} \boldsymbol{u}+L_{i}(\boldsymbol{y}-\widehat{\boldsymbol{y}}) \\
\widehat{\boldsymbol{y}} & =C_{i} \widehat{\boldsymbol{x}}
\end{aligned}
\end{aligned}
$$

for $i=1,2, \ldots, r$ where

- $\widehat{\boldsymbol{x}}$ represents the estimated system states.

- $y$ represents the output of the system.

- $L_{i}, i=1,2, \ldots, r$ are the observer gains.

The observer in equation (2-34) can be represented as:

$$
\begin{aligned}
\dot{\hat{\boldsymbol{x}}} & =\sum_{i=1}^{r} h_{i}(\widehat{\boldsymbol{z}})\left(A_{i} \widehat{\boldsymbol{x}}+B_{i} \boldsymbol{u}+L_{i}(\boldsymbol{y}-\widehat{\boldsymbol{y}})\right) \\
\widehat{\boldsymbol{y}} & =\sum_{i=1}^{r} h_{i}(\widehat{\boldsymbol{z}}) C_{i} \widehat{\boldsymbol{x}}
\end{aligned}
$$

Two cases have been discussed in [19] to find the conditions for stability.

Case A: When the antecedent variables are independent of the state variables. This case is relatively simple in terms of analysis.

Case B: When the antecedent variables are dependent on the state variables (the state variables being estimated by the fuzzy observer)

In case $\mathrm{A}$, the observer (2-37) is represented as

$$
\begin{aligned}
\dot{\hat{\boldsymbol{x}}} & =\sum_{i=1}^{r} h_{i}(\boldsymbol{z})\left(A_{i} \widehat{\boldsymbol{x}}+B_{i} \boldsymbol{u}+L_{i}(\boldsymbol{y}-\widehat{\boldsymbol{y}})\right) \\
\widehat{\boldsymbol{y}} & =\sum_{i=1}^{r} h_{i}(\boldsymbol{z}) C_{i} \widehat{\boldsymbol{x}}
\end{aligned}
$$

where $\boldsymbol{z}$ is a measured vector.

Substituting the estimated output equation (2-36) in (2-35), we get

$$
\dot{\widehat{\boldsymbol{x}}}=\sum_{i=1}^{r} h_{i}(\boldsymbol{z}) A_{i} \widehat{\boldsymbol{x}}+\sum_{i=1}^{r} h_{i}(\boldsymbol{z}) B_{i} \boldsymbol{u}+\sum_{i=1}^{r} \sum_{j=1}^{r} h_{i}(\boldsymbol{z}) h_{j}(\boldsymbol{z}) L_{i} C_{j}(\boldsymbol{x}(t)-\widehat{\boldsymbol{x}}(t))
$$


Considering the error $\boldsymbol{e}(t)=\boldsymbol{x}(t)-\widehat{\boldsymbol{x}}(t)$, the error dynamics of the observer is

$$
\dot{\boldsymbol{e}}(t)=\sum_{i=1}^{r} \sum_{j=1}^{r} h_{i}(\boldsymbol{z}) h_{j}(\boldsymbol{z})\left(A_{i}-L_{i} C_{j}\right) \boldsymbol{e}(t)
$$

The observer and the state estimation error equations for case B and their analysis is complex and beyond the scope of this thesis. LMI conditions to guarantee stability of the observers are presented in [20]. Further details on observers are presented in Chapter 5 which deals specifically with adaptive observer design.

The TS fuzzy models, the TS fuzzy regulators and the observers discussed above assume that an exact model of the nonlinear system is available. The uncertainties in the system modelling are not considered. Uncertain TS fuzzy models, robust fuzzy state feedback controllers and adaptive observers that estimate uncertainty are all discussed in detail in Chapters 4 and 5. However, a brief introduction to their mathematical representation will be provided in the next section. The next section builds on the concepts presented till now, and presents the problem addressed in this thesis in a mathematical form.

\section{2-7 Problem statement}

We deal with design of adaptive observers and robust state feedback controllers for uncertain TS fuzzy systems in this thesis, as mentioned in the first chapter. In particular we investigate uncertainties in the nonlinear system that can be viewed as a constant additive component to the state matrices of a nominal TS fuzzy model. These kind of uncertainties can possibly be seen as constant yet unmodelled dynamics. As an example, we can consider unmodelled friction. In this section, we start with a TS fuzzy model with a specific uncertainty distribution structure in the local linear models and outline the mathematical details of the problem addressed in this thesis.

Consider a nonlinear system represented by the TS fuzzy model:

$$
\begin{aligned}
& R_{i}: \text { If } z_{1} \text { is } N_{i}^{1} \text { and } z_{2} \text { is } N_{i}^{2}, \ldots \text { and } z_{p} \text { is } N_{i}^{p} \text {, then } \\
& \qquad \begin{aligned}
\dot{\boldsymbol{x}} & =A_{i} \boldsymbol{x}+B_{i} \boldsymbol{u}+M_{i} A_{\delta i} \boldsymbol{x} \\
\boldsymbol{y} & =C \boldsymbol{x}
\end{aligned}
\end{aligned}
$$

for $i=1,2, \ldots, r$, where

- $r$ is the number of rules.

- $\boldsymbol{x}$ is the state vector, $\boldsymbol{u}$ is the input vector, $\boldsymbol{y}$ is the output vector.

- $n_{x}$ is the dimension of $\boldsymbol{x}$ and $n_{u}$ is the dimension of $\boldsymbol{u}$.

- $\boldsymbol{x} \in \mathbb{R}^{n_{x}}, \boldsymbol{u} \in \mathbb{R}^{n_{u}}$, and $\boldsymbol{y} \in \mathbb{R}^{n_{y}}$.

- $\boldsymbol{z}=\left[z_{1}, z_{2} \ldots z_{p}\right]^{T}$ is the vector of scheduling variables. 
- $A_{i} \in \mathbb{R}^{n_{x} \times n_{x}}, B_{i} \in \mathbb{R}^{n_{x} \times n_{u}}, i=1,2, \ldots, r$ are known state and input matrices of the individual linear models that are combined to represent the nonlinear system.

- $C$ is the common output matrix for the local models.

- $M_{i}, i=1,2, \ldots, r$, are the uncertainty distribution matrices.

- $A_{\delta i}, i=1,2, \ldots, r$, are the blocks representing uncertainties in the models ${ }^{1}$.

- The values of the elements in the uncertainty blocks $A_{\delta i}$ are unknown, but their norm is bounded, i.e., there exists a known $\mu_{i} \in \mathbb{R}$ so that $\left\|A_{\delta i}\right\| \leq \mu_{i}$, for all $i=1,2, \ldots, r$ $\left(\left\|A_{\delta i}\right\|\right.$ represents the 2 -norm of the matrix $\left.A_{\delta i}\right)$.

Equation (2-40) can be rewritten as

$$
\begin{aligned}
\dot{\boldsymbol{x}} & =\sum_{i=1}^{r} h_{i}(\boldsymbol{z})\left(A_{i} \boldsymbol{x}+B_{i} \boldsymbol{u}+M_{i} A_{\delta i} \boldsymbol{x}\right) \\
\boldsymbol{y} & =C \boldsymbol{x}
\end{aligned}
$$

Constant uncertainties in the state matrices of the local linear models of an uncertain TS fuzzy model (2-41) can be estimated using the adaptive observer developed in [1], if the uncertainties are observable. As long as the uncertainty observability condition is satisfied, the observer can estimate both unmeasured states and uncertainties. The condition to ensure observability of the uncertainty is detailed in Chapter 5. In this thesis, all the states of the system are considered to be measured and we concentrate on the use of adaptive observer concept for uncertainty estimation purposes only. Thus, we expect to get a better TS fuzzy model of the system by using an adaptive observer. The adaptive observer used in the design is of the form

$$
\begin{aligned}
\dot{\hat{\boldsymbol{x}}} & =\sum_{i=1}^{r} h_{i}(\boldsymbol{z})\left(A_{i} \widehat{\boldsymbol{x}}+B_{i} \boldsymbol{u}+M_{i} \widehat{A}_{\delta i} \widehat{\boldsymbol{x}}+L_{i}(\boldsymbol{y}-\widehat{\boldsymbol{y}})\right) \\
\widehat{\boldsymbol{y}} & =\sum_{i=1}^{r} h_{i}(\boldsymbol{z})\left(C_{i} \widehat{\boldsymbol{x}}\right) \\
\dot{\hat{A}}_{\delta i} & =f_{i}\left(\widehat{A}_{\delta i}, \boldsymbol{h}(\boldsymbol{z}), \widehat{\boldsymbol{x}}, \boldsymbol{y}\right) \quad i=1,2, \ldots, r
\end{aligned}
$$

with $L_{i}, i=1,2, \ldots, r$, being the observer gain matrices and $f_{i}, i=1,2, \ldots, r$ being the update law for $\widehat{A}_{\delta i}, i=1,2, \ldots, r$ such that the estimation errors $(\boldsymbol{x}-\widehat{\boldsymbol{x}})$ and $\left(A_{\delta i}-\widehat{A}_{\delta i}\right)$ converge asymptotically to zero. In the design of the adaptive observer, the norm of the uncertainty blocks is considered to be bounded by a known value $\mu_{\max }$. We study the effect of increasing the value of $\mu_{\max }$ on the rate of convergence of uncertainty estimation error through the investigation of simulation results. The design of adaptive observer and the results are the subject of detailed analysis in Chapter 5 .

Our goal is to obtain a better model of the system and evaluate any improvement that is achieved in the control of the uncertain TS fuzzy model by using the uncertainty estimates.

\footnotetext{
${ }^{1}$ The uncertainty in the state matrices are $\Delta A_{i}$. They are represented by the product of $M_{i}$ and $A_{\delta i}$, and this product is referred to as uncertainty distribution structure.
} 
To perform this analysis, we need a robust state feedback controller design for the same uncertainty distribution structure as in the adaptive observer. However, in existing literature $[5,8]$, robust state feedback controller design for uncertainty distribution structure of a different kind than the one presented in equation (2-40) are considered i.e., $\Delta A_{i}=D_{a i} \Delta_{a i} E_{a i}$. Hence in this thesis, a robust state feedback controller is designed for $\Delta A_{i}=M_{i} A_{\delta i}$. Based on Lyapunov's direct method, LMI conditions are derived to guarantee stability of the closed loop system in the presence of uncertainty $\left(\Delta A_{i}=M_{i} A_{\delta i}\right)$ in the state matrices of the local linear models. The design of the robust state feedback controller considers the norm of the uncertainty block in every local linear model to be bounded by a known value $\mu_{i}$. The design of the robust state feedback controller will be the subject of detailed analysis in Chapter 4.

The uncertainty estimates obtained by the adaptive observer are in the sequel used in the robust state feedback controller design. The analysis of redesign of robust controller using an updated model is discussed in Chapter 6 .

This chapter presented an overview of fuzzy systems and TS fuzzy models were described in detail. General stability concepts were outlined followed by stability analysis of continuous time TS fuzzy systems. A brief overview of TS fuzzy regulators and observers was presented. These concepts provide the necessary background to present the mathematical details of the problem addressed in this thesis.

To illustrate the application of robust state feedback controller and the adaptive observer concepts, three candidate examples are investigated. TS fuzzy models of each example is constructed using sector nonlinearity and is discussed in Appendix B. Out of the three examples, the 2-DOF robot arm model is chosen and construction of its TS model is detailed in the following chapter. 


\section{Chapter 3}

\section{Fuzzy modelling of 2-DOF robot arm}

In this chapter, we construct a Takagi-Sugeno (TS) fuzzy model for a 2-DOF robot arm based on the sector nonlinearity approach. The interested reader should refer to Appendices A and $\mathrm{B}$ for the sector nonlinearity concept and examples of construction of TS fuzzy models using this approach.

The first section in this chapter presents the nonlinear model of the robot arm. Considering linear models for the DC motors at the robot arm joints, parameters of the models are estimated using input-output data generated through experiments on a laboratory scale 2DOF robot arm. This is detailed in Section 3-2. The parameters obtained for the individual joints are then used in the nonlinear model to find the most probable values of unknown parameters by nonlinear optimization. The simulation results are compared with the outputs from the plant (i.e., the laboratory scale 2 - DOF robot arm) in Section 3-3. Simplification of the nonlinear model and construction of a TS fuzzy model for the simplified nonlinear model is presented in Sections 3-4 and 3-5. Section 3-5 ends the chapter with the validation of the TS fuzzy model.

\section{3-1 Nonlinear model of a 2-DOF robot arm}

The nonlinear model of the 2 -DOF robot arm operating in the horizontal plane is:

$$
M(\theta) \ddot{\theta}+C(\theta, \dot{\theta}) \dot{\theta}=\tau
$$

where

$$
\begin{aligned}
M(\theta) & =\left[\begin{array}{cc}
P_{1}+P_{2}+2 P_{3} \cos \theta_{2} & P_{2}+P_{3} \cos \theta_{2} \\
P_{2}+P_{3} \cos \theta_{2} & P_{2}
\end{array}\right] \\
C(\theta, \dot{\theta}) & =\left[\begin{array}{cc}
b_{1}-P_{3} \dot{\theta_{2}} \sin \theta_{2} & -P_{3}\left(\dot{\theta_{1}}+\dot{\theta_{2}}\right) \sin \theta_{2} \\
-P_{3} \dot{\theta_{2}} \sin \theta_{2} & b_{2}
\end{array}\right]
\end{aligned}
$$

The parameters in the equation above are described in Section B-3 of Appendix B. 
The values of the parameters in equations (3-2) and (3-3) are estimated and model verification is performed. The details of the parameter estimation are presented in the following two sections.

\section{3-2 Modelling of the robot arm joints}

The laboratory setup of the 2-DOF robot arm contains two joints actuated by DC motors. By means of SISO input-output data generating experiments and using MATLAB's system identification toolbox, linear models of the DC motors are obtained. One of the main assumptions in the identification is that the robot arm is expected to work at low frequencies, practically less than $5 \mathrm{~Hz}$. Hence the bandwidth of the input signals is limited to $5 \mathrm{~Hz}$. This motivates the selection of a sampling rate of 10 times the system bandwidth, which is $50 \mathrm{~Hz}$ (sampling time of $0.02 \mathrm{~s}$ ). Two input voltages $u_{1}$ and $u_{2}$ are available to actuate the joints. To obtain the DC motor models, the system is excited with a random binary signal (RBS) of amplitude +/-0.1 normalized voltage to maintain the robot arm in a safe operating region. During all experiments it is ensured that the input signals to the two joints are uncorrelated. SISO operation of the joints is performed i.e., only one joint is actuated and angular position of the corresponding link is noted during the experiments.

The DC motor can be physically modelled by combining the equations for the electrical part and the mechanical part of the system i.e.,

$$
\begin{aligned}
I \ddot{\theta}+b \dot{\theta} & =K_{t} i \\
L \frac{d i}{d t}+R i & =u-K_{e} \dot{\theta}
\end{aligned}
$$

where

- $K=K_{t}=K_{e}$

- $K_{t}$ is the armature constant in the relation $\tau=K_{t} i$. ( $\tau$ is the torque and $i$ is the motor current.)

- $K_{e}$ is the motor constant in the relation $e_{b}=K_{e} \dot{\theta}$. ( $e_{b}$ is the back e.m.f.)

- $u$ is the input voltage.

- $R$ is the resistance in the motor armature and $L$ is the armature inductance.

- $I$ is the inertia of the load.

- $b$ is the damping in the mechanical system.

Considering small inductances, we have

$$
i=\frac{u-K \dot{\theta}}{R}
$$

and

$$
\ddot{\theta}+\frac{\left(b+\frac{K^{2}}{R}\right)}{I} \dot{\theta}=\frac{K}{R I} u
$$


Equation (3-6) gives the model of the DC motor. The transfer function of the model (3-6) is

$$
\frac{\theta(s)}{U(s)}=\frac{k_{I}}{s\left(s+b_{I}\right)}
$$

where $k_{I}=\frac{K}{R I}$ and $b_{I}=\frac{\left(b+\frac{K^{2}}{R}\right)}{I}$.

Based on the experiments with RBS inputs, the models for the joints 1 and 2 (represented as $J_{1}$ and $J_{2}$ ) based on (3-6) are found to be

$$
\frac{\theta_{1}(s)}{U_{1}(s)}=\frac{147.97}{s(s+28.74)}
$$

$\left(k_{I 1}=147.97\right.$ and $\left.b_{I 1}=28.74\right)$ and

$$
\frac{\theta_{2}(s)}{U_{2}(s)}=\frac{156.97}{s(s+31.99)}
$$

$\left(k_{I 2}=156.97\right.$ and $\left.b_{I 2}=31.99\right)$

Figures 3-1 and 3-2 compare the measured angle values with the simulated outputs from the identified model for $J_{1}$ and $J_{2}$, respectively.
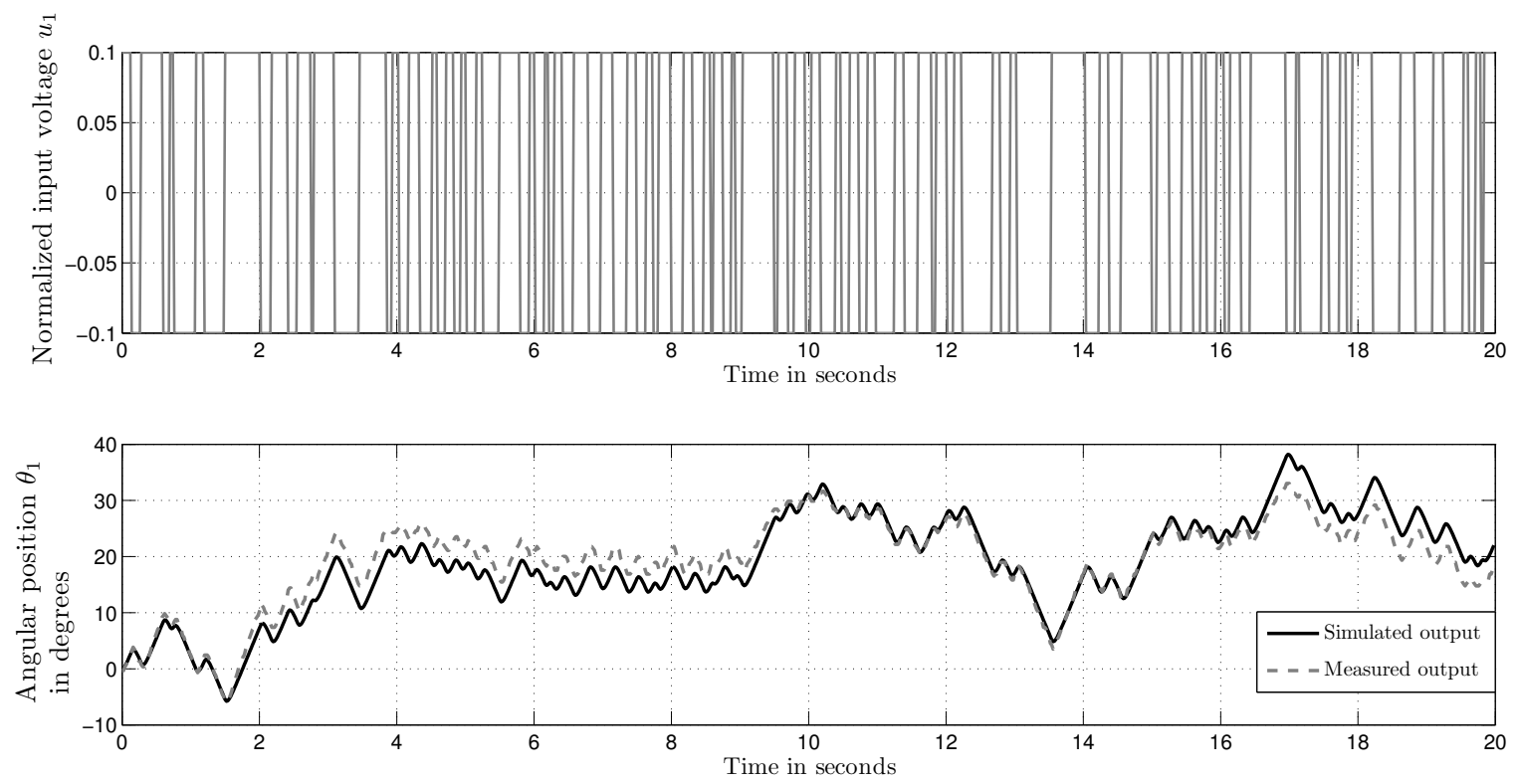

Figure 3-1: Simulation results for identified $J_{1}$ model.

\section{3-3 Finding the parameters of the nonlinear 2-DOF robot arm}

The nonlinear model of the 2-DOF robot arm is

$$
M(\theta) \ddot{\theta}+C(\theta, \dot{\theta}) \dot{\theta}=\tau
$$



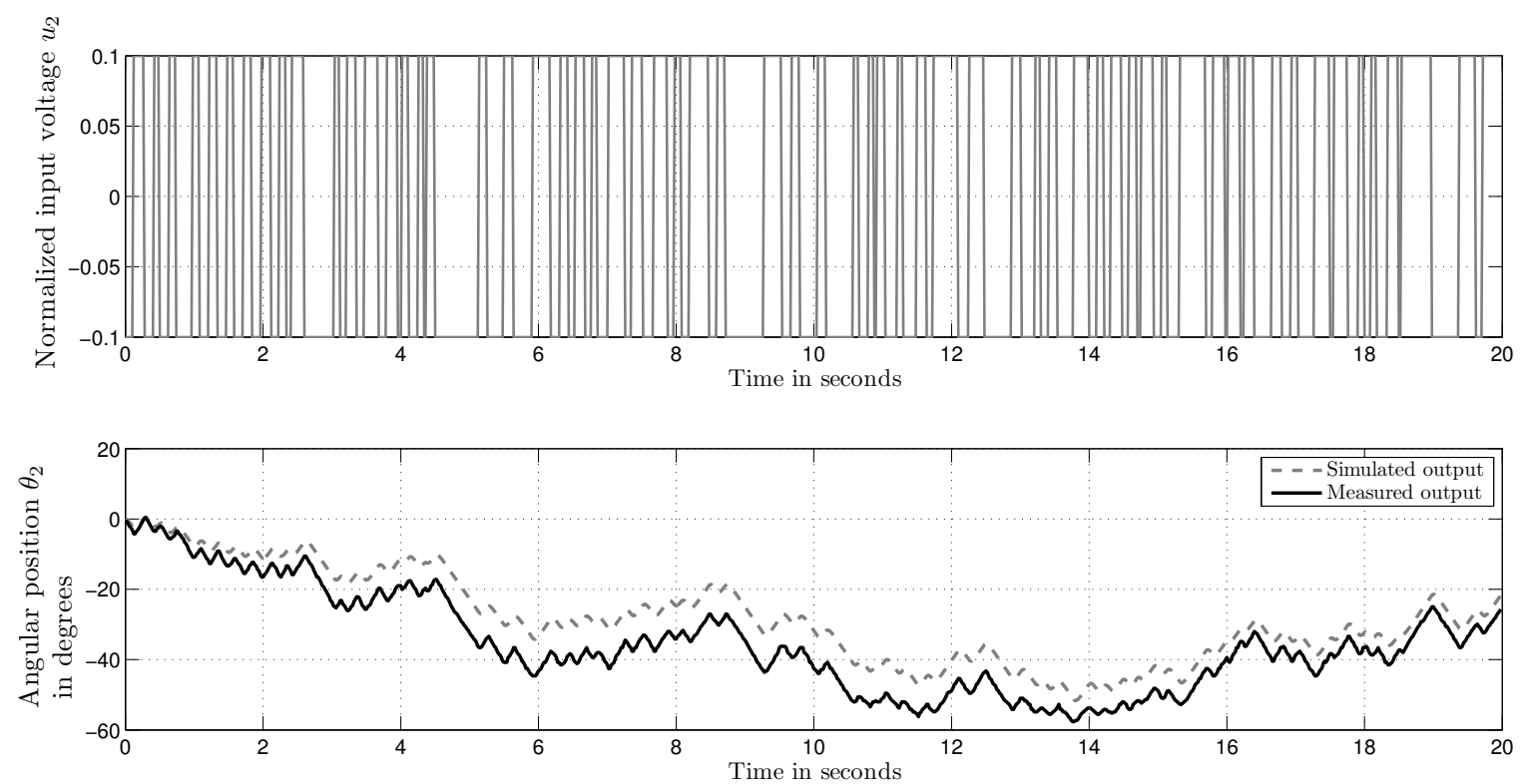

Figure 3-2: Simulation results for identified $J_{2}$ model.

where

- $\theta=\left[\begin{array}{ll}\theta_{1} & \theta_{2}\end{array}\right]^{T}$ and $\tau=\left[\begin{array}{ll}\tau_{1} & \tau_{2}\end{array}\right]^{T}$

- $\tau_{1}, \tau_{2}$ are the control inputs as the two joints (torque inputs).

- $\theta_{1}, \theta_{2}, \dot{\theta_{1}}, \dot{\theta_{2}}$ are measured positions and angular velocities.

- $M(\theta)$ is the mass matrix.

- $C(\theta, \dot{\theta})$ is the Coriolis and centrifugal force matrix.

The mass and the Coriolis matrices are

$$
\begin{aligned}
M(\theta) & =\left[\begin{array}{cc}
P_{1}+P_{2}+2 P_{3} \cos \theta_{2} & P_{2}+P_{3} \cos \theta_{2} \\
P_{2}+P_{3} \cos \theta_{2} & P_{2}
\end{array}\right] \\
C(\theta, \dot{\theta}) & =\left[\begin{array}{cc}
b_{1}-P_{3} \dot{\theta_{2}} \sin \theta_{2} & -P_{3}\left(\dot{\theta_{1}}+\dot{\theta_{2}}\right) \sin \theta_{2} \\
-P_{3} \dot{\theta}_{2} \sin \theta_{2} & b_{2}
\end{array}\right]
\end{aligned}
$$

where

- $P_{1}=m_{1} c_{1}^{2}+m_{2} l_{1}^{2}+I_{1}$

- $P_{2}=m_{2} c_{2}^{2}+I_{2}$

- $P_{3}=m_{2} l_{1} c_{2}$ 
- $l_{1}$ and $l_{2}$ are the lengths of the first and the second link, respectively.

- $m_{1}$ and $m_{2}$ are the masses of the first and the second link, respectively.

- $I_{1}$ and $I_{2}$ are the inertias of the first and the second link, respectively.

- $c_{1}$ and $c_{2}$ are the centres of mass of the first and the second link, respectively.

- $b_{1}$ and $b_{2}$ are the damping in the first and the second joint, respectively.

The values of $k_{I 1}, k_{I 2}, b_{I 1}$ and $b_{I 2}$ that are obtained from the modelling of the DC motors are used in the above model. With the values of parameters $m_{1}=0.45 \mathrm{~kg}, m_{2}=0.125 \mathrm{~kg}$, $l_{1}=0.4 \mathrm{~m}, l_{2}=0.1 \mathrm{~m}$, we get the inertias for the links to be $I_{1}=0.0240 \mathrm{~kg} \mathrm{~m}^{2}$ and $I_{2}=$ $0.000416 \mathrm{~kg} \mathrm{~m}^{2}$. Multiplying the values $k_{I 1}, k_{I 2}, b_{I 1}$ and $b_{I 2}$ with the values of inertias of respective links we get estimates for $k_{m 1}=6.59, k_{m 2}=1.51, b_{1}=1.22$ and $b_{2}=0.24$ where $k_{m 1}$ and $k_{m 2}$ are the equivalent of $K / R$ in the model (3-6). The values of $k_{m 1}$ and $k_{m 2}$ can be considered as motor gains and result in representing the input to the system as input $u_{1}$ multiplied by gain $k_{m 1}$ and $u_{2}$ multiplied by $k_{m 2}$ respectively. MIMO experiments are performed with inputs being simultaneously applied to both the joints and input-output data is collected. Nonlinear optimization (using 'Isqnonlin' function of MATLAB) is performed to estimate the values of the parameters $P_{1}, P_{2}$ and $P_{3}$. These values are found to be $P_{1}=0.0191$, $P_{2}=0.00039$ and $P_{3}=0.00000967$. With the parameter values obtained in this section, a TS fuzzy model is created for the 2-DOF robot arm. This is detailed in the next section.

With the parameters of the nonlinear model as determined by nonlinear optimization and linear parameter estimation of the individual joints, Figures 3-3 and 3-4 compare the measured and the simulation result of the nonlinear model. A multisine signal with frequency upto $3 \mathrm{~Hz}$ is used for validation purposes. The difference in the outputs can be attributed to unmodelled dynamics (for e.g. unmodelled friction or the inexact value of damping co-efficient).

A TS fuzzy model for the nonlinear model of the 2-DOF robot arm would require 64 rules due to the presence of 6 nonlinearities (see Appendix B). A simplified nonlinear model can be obtained by neglecting the Coriolis and centrifugal forces. A 4 rule fuzzy model is constructed for the 2-DOF robot arm in the following section. For the development of the fuzzy state feedback controller for the robot arm, the measurements of the 2 angular positions and 2 angular velocities are required and hence all the states are considered to be measured. To test the results obtained in this thesis on the laboratory setup, linear observers may be designed for each individual joint to obtain estimates of the angular velocities. These estimates may be used instead of measured values of velocities.

\section{3-4 Simplification of the nonlinear model}

A simplification of the 2-DOF robot arm nonlinear model is considered to obtain a fuzzy model with a smaller number of rules. The simplification is presented in this section.

Neglecting Coriolis and centrifugal forces and considering movement in the horizontal plane, the model of the 2-DOF robot arm can be represented as

$$
M(\theta) \ddot{\theta}+C(\theta, \dot{\theta}) \dot{\theta}=\tau
$$



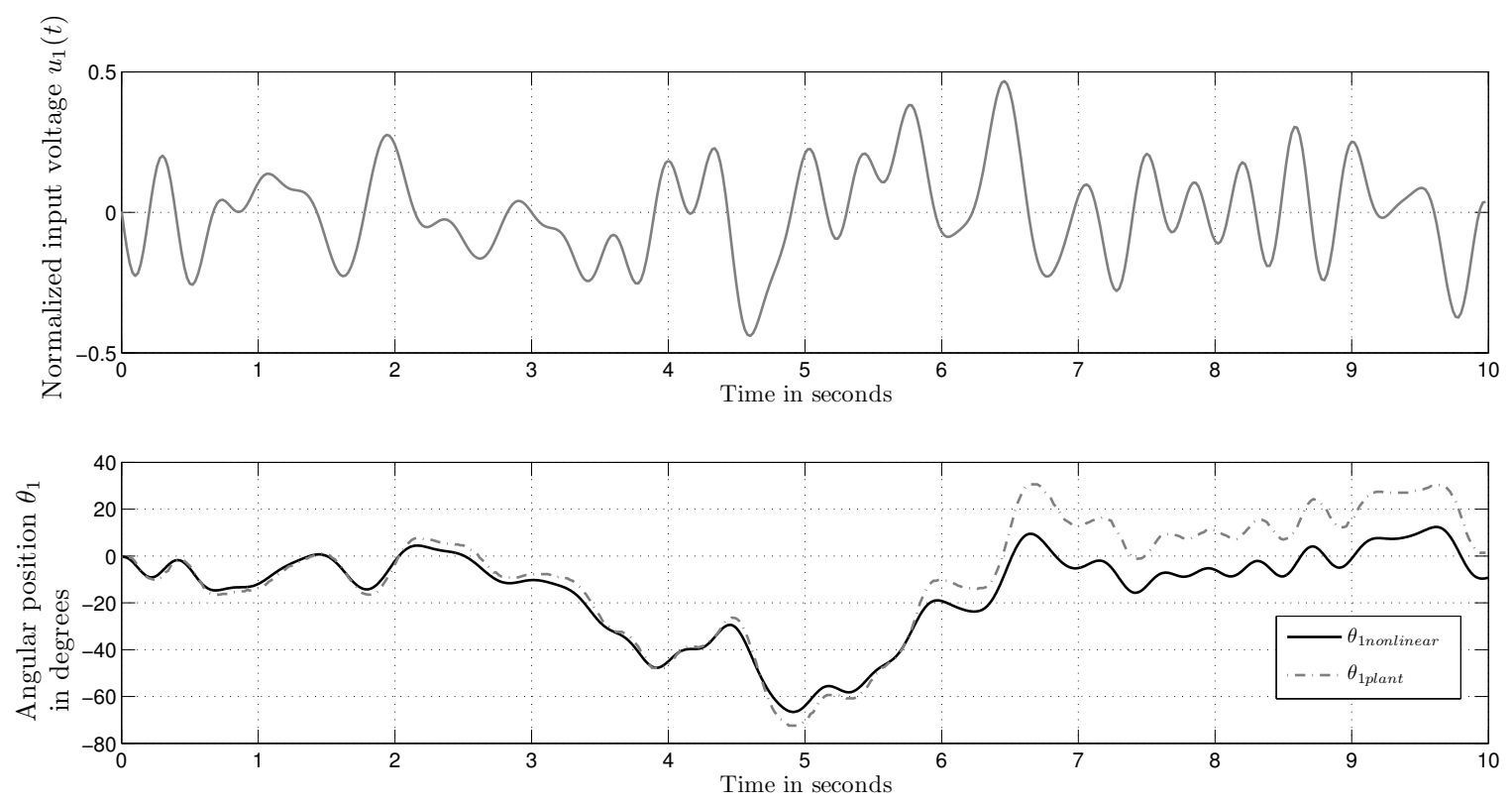

Figure 3-3: Comparison of measured and simulated output from the nonlinear model $-\theta_{1}$.

where $C(\theta, \dot{\theta})=\left[\begin{array}{cc}b_{1} & 0 \\ 0 & b_{2}\end{array}\right]$.

Equation (3-13) can be represented in the form of state and input matrices as follows.

$$
\dot{\boldsymbol{x}}=\left[\begin{array}{cc}
-M^{-1} C & 0 \\
I & 0
\end{array}\right] \boldsymbol{x}+\left[\begin{array}{c}
M^{-1} \\
\mathbf{0}
\end{array}\right] \tau
$$

where

$\boldsymbol{x}=\left[\begin{array}{llll}\dot{\theta}_{1} & \dot{\theta}_{2} & \theta_{1} & \theta_{2}\end{array}\right]^{T}$

For the ease of notation, the dependency of $M$ on $\theta$ is not explicitly noted in the above equation. The Coriolis matrix $C$ is not dependent on $\theta$ or $\dot{\theta}$ since Coriolis and centrifugal forces are neglected and hence $C$ is independent of $\theta$ and $\dot{\theta}$.

Substituting the values of $M, C$, and representing the torques as a product of motor gain and input voltage in the above equation, we obtain the state-space model

$$
\dot{\boldsymbol{x}}=A\left(\theta_{2}\right) \boldsymbol{x}+B\left(\theta_{2}\right) \boldsymbol{u}
$$

where 

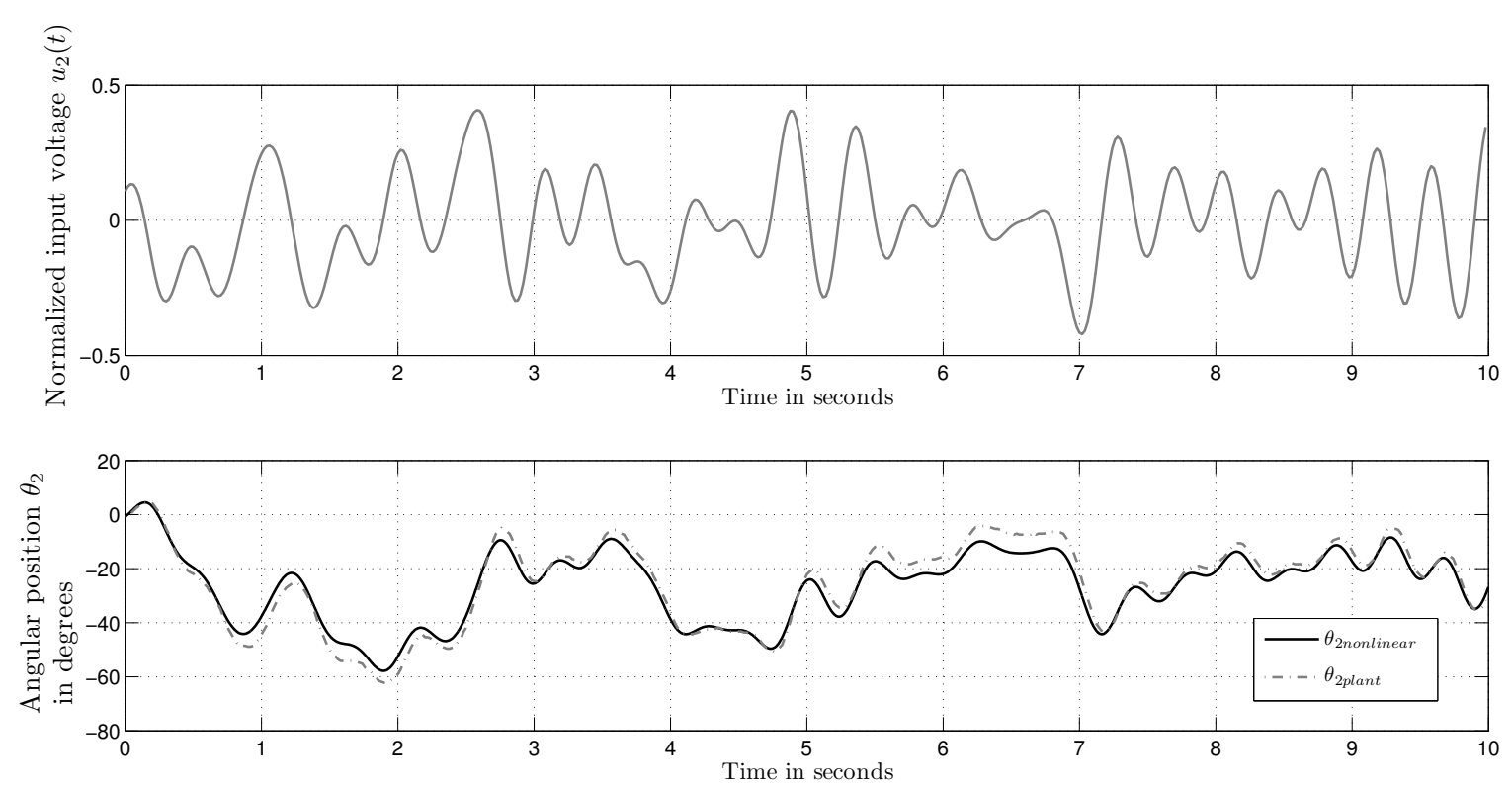

Figure 3-4: Comparison of measured and simulated output from the nonlinear model $-\theta_{2}$.

$$
A\left(\theta_{2}\right)=\left[\begin{array}{cccc}
\frac{P_{2} b_{1}}{P_{3}^{2} \cos \theta_{2}{ }^{2}-P_{1} P_{2}} & -\frac{b_{2}\left(P_{2}+P_{3} \cos \left(\theta_{2}\right)\right.}{P_{3}^{2} \cos \theta_{2}{ }^{2}-P_{1} P_{2}} & 0 & 0 \\
-\frac{b_{1}\left(P_{2}+P_{3} \cos \left(\theta_{2}\right)\right.}{P_{3}^{2} \cos \theta_{2}{ }^{2}-P_{1} P_{2}} & \frac{b_{2}\left(P_{1}+P_{2}+2 P_{3} \cos \left(\theta_{2}\right)\right.}{P_{3}^{2} \cos \theta_{2}{ }^{2}-P_{1} P_{2}} & 0 & 0 \\
1 & 0 & 0 & 0 \\
0 & 1 & 0 & 0
\end{array}\right]
$$

and

$$
B\left(\theta_{2}\right)=\left[\begin{array}{cc}
\frac{-P_{2} k_{m 1}}{P_{3}^{2} \cos \theta_{2}^{2}-P_{1} P_{2}} & \frac{k_{m 2}\left(P_{2}+P_{3} \cos \left(\theta_{2}\right)\right.}{P_{3}^{2} \cos \theta_{2}^{2}-P_{1} P_{2}} \\
\frac{k_{m 1}\left(P_{2}+P_{3} \cos \left(\theta_{2}\right)\right.}{P_{3}^{2} \cos \theta_{2}{ }^{2}-P_{1} P_{2}} & -\frac{k_{m 2}\left(P_{1}+P_{2}+2 P_{3} \cos \left(\theta_{2}\right)\right.}{P_{3}^{2} \cos \theta_{2}^{2}-P_{1} P_{2}} \\
0 & 0 \\
0 & 0
\end{array}\right]
$$

Given the above simplified nonlinear model for the 2-DOF robot arm, the next section presents the construction of the corresponding TS fuzzy model. 


\section{3-5 TS fuzzy model for the simplified 2-DOF robot arm}

Considering the scheduling variables

$$
z_{1}=\frac{1}{P_{3}^{2} \cos \left(\theta_{2}\right)^{2}-P_{1} P_{2}}
$$

and

$$
z_{2}=\frac{\cos \left(\theta_{2}\right)}{P_{3}{ }^{2} \cos \left(\theta_{2}\right)^{2}-P_{1} P_{2}}
$$

we have the matrices $A$ and $B$ given by (3-16) and (3-17) in terms of the nonlinearities as follows (refer Appendix B for details).

$$
A\left(z_{1}, z_{2}\right)=\left[\begin{array}{cccc}
P_{2} b_{1} z_{1} & -P_{2} b_{2} z_{1}-P_{3} b_{2} z_{2} & 0 & 0 \\
-P_{2} b_{1} z_{1}-P_{3} b_{1} z_{2} & b_{2} z_{1}\left(P_{1}+P_{2}\right)+2 P_{3} b_{2} z_{2} & 0 & 0 \\
1 & 0 & 0 & 0 \\
0 & 1 & 0 & 0
\end{array}\right]
$$

and

$$
B\left(z_{1}, z_{2}\right)=\left[\begin{array}{cc}
-P_{2} k_{m 1} z_{1} & k_{m 2}\left(P_{2} z_{1}+P_{3} z_{2}\right) \\
k_{m 1}\left(P_{2} z_{1}+P_{3} z_{2}\right) & -k_{m 2}\left(2 P_{3} z_{2}+z_{1}\left(P_{1}+P_{2}\right)\right) \\
0 & 0 \\
0 & 0
\end{array}\right]
$$

With the two nonlinearities $z_{1}$ and $z_{2}$, we can construct 4 rules to represent the system. $R_{1}$ : If $z_{1}$ is $N_{1}^{1}$ and $z_{2}$ is $N_{1}^{2}$ then

$$
\begin{aligned}
\dot{\boldsymbol{x}} & =A_{1} \boldsymbol{x}+B_{1} \boldsymbol{u} \\
\boldsymbol{y} & =C_{1} \boldsymbol{x}
\end{aligned}
$$

$R_{2}:$ If $z_{1}$ is $N_{2}^{1}$ and $z_{2}$ is $N_{2}^{2}$ then

$$
\begin{aligned}
\dot{\boldsymbol{x}} & =A_{2} \boldsymbol{x}+B_{2} \boldsymbol{u} \\
\boldsymbol{y} & =C_{2} \boldsymbol{x}
\end{aligned}
$$

$R_{3}$ : If $z_{1}$ is $N_{3}^{1}$ and $z_{2}$ is $N_{3}^{2}$ then

$$
\begin{aligned}
\dot{\boldsymbol{x}} & =A_{3} \boldsymbol{x}+B_{3} \boldsymbol{u} \\
\boldsymbol{y} & =C_{3} \boldsymbol{x}
\end{aligned}
$$


$R_{4}$ : If $z_{1}$ is $N_{4}^{1}$ and $z_{2}$ is $N_{4}^{2}$ then

$$
\begin{aligned}
\dot{\boldsymbol{x}} & =A_{4} \boldsymbol{x}+B_{4} \boldsymbol{u} \\
\boldsymbol{y} & =C_{4} \boldsymbol{x}
\end{aligned}
$$

where

$$
A_{1}=\left[\begin{array}{cccc}
P_{2} b_{1} z_{1 \text { min }} & -P_{2} b_{2} z_{1 \text { min }}-P_{3} b_{2} z_{2 m i n} & 0 & 0 \\
-P_{2} b_{1} z_{1 \text { min }}-P_{3} b_{1} z_{2 \min } & b_{2} z_{1 \min }\left(P_{1}+P_{2}\right)+2 P_{3} b_{2} z_{2 m i n} & 0 & 0 \\
1 & 0 & 0 & 0 \\
0 & 1 & 0 & 0
\end{array}\right]
$$

and

$$
\begin{aligned}
& B_{1}=\left[\begin{array}{cc}
-P_{2} k_{m 1} z_{1 m i n} & k_{m 2}\left(P_{2} z_{1 m i n}+P_{3} z_{2 m i n}\right) \\
k_{m 1}\left(P_{2} z_{1 m i n}+P_{3} z_{2 m i n}\right) & -k_{m 2}\left(2 P_{3} z_{2 m i n}+z_{1 m i n}\left(P_{1}+P_{2}\right)\right) \\
0 & 0 \\
0 & 0
\end{array}\right] \\
& A_{2}=\left[\begin{array}{cccc}
P_{2} b_{1} z_{1 \min } & -P_{2} b_{2} z_{1 \min }-P_{3} b_{2} z_{2 \max } & 0 & 0 \\
-P_{2} b_{1} z_{1 \min }-P_{3} b_{1} z_{2 \max } & b_{2} z_{1 \min }\left(P_{1}+P_{2}\right)+2 P_{3} b_{2} z_{2 \max } & 0 & 0 \\
1 & 0 & 0 & 0 \\
0 & 1 & 0 & 0
\end{array}\right]
\end{aligned}
$$

and

$$
B_{2}=\left[\begin{array}{cc}
-P_{2} k_{m 1} z_{1 \min } & k_{m 2}\left(P_{2} z_{1 \min }+P_{3} z_{2 \max }\right) \\
k_{m 1}\left(P_{2} z_{1 \min }+P_{3} z_{2 \max }\right) & -k_{m 2}\left(2 P_{3} z_{2 \max }+z_{1}\left(P_{1}+P_{2}\right)\right) \\
0 & 0 \\
0 & 0
\end{array}\right]
$$




$$
A_{3}=\left[\begin{array}{cccc}
P_{2} b_{1} z_{1 \max } & -P_{2} b_{2} z_{1 \max }-P_{3} b_{2} z_{2 \min } & 0 & 0 \\
-P_{2} b_{1} z_{1 \max }-P_{3} b_{1} z_{2 \min } & b_{2} z_{1 \max }\left(P_{1}+P_{2}\right)+2 P_{3} b_{2} z_{2 \min } & 0 & 0 \\
1 & 0 & 0 & 0 \\
0 & 1 & 0 & 0
\end{array}\right]
$$

and

$$
\begin{aligned}
B_{3} & =\left[\begin{array}{ccc}
-P_{2} k_{m 1} z_{1 \max } & k_{m 2}\left(P_{2} z_{1 \max }+P_{3} z_{2 \min }\right) & \\
k_{m 1}\left(P_{2} z_{1 \max }+P_{3} z_{2 \min }\right) & -k_{m 2}\left(2 P_{3} z_{2 \min }+z_{1 \max }\left(P_{1}+P_{2}\right)\right) \\
0 & 0 & \\
0 & 0 &
\end{array}\right] \\
A_{4} & =\left[\begin{array}{cccc}
P_{2} b_{1} z_{1 \max } & -P_{2} b_{2} z_{1 \max }-P_{3} b_{2} z_{2 \max } & 0 & 0 \\
-P_{2} b_{1} z_{1 \max }-P_{3} b_{1} z_{2 \max } & b_{2} z_{1 \max }\left(P_{1}+P_{2}\right)+2 P_{3} b_{2} z_{2 \max } & 0 & 0 \\
1 & 0 & 0 & 0 \\
0 & 1 & 0 & 0
\end{array}\right]
\end{aligned}
$$

and

$$
B_{4}=\left[\begin{array}{cc}
-P_{2} k_{m 1} z_{1 \max } & k_{m 2}\left(P_{2} z_{1 \max }+P_{3} z_{2 \max }\right) \\
k_{m 1}\left(P_{2} z_{1 \max }+P_{3} z_{2 \max }\right) & -k_{m 2}\left(2 P_{3} z_{2 \max }+z_{1 \max }\left(P_{1}+P_{2}\right)\right) \\
0 & 0 \\
0 & 0
\end{array}\right]
$$

$C_{1}=C_{2}=C_{3}=C_{4}=\left[\begin{array}{cccc}1 & 0 & 0 & 0 \\ 0 & 1 & 0 & 0 \\ 0 & 0 & 1 & 0 \\ 0 & 0 & 0 & 1\end{array}\right]$

As seen from the model in Section 3-5, the two nonlinear terms in the state and the input matrices depend on the angle $\theta_{2}$ only. From laboratory experiments, it has been found that the maximum and minimum values that $\theta_{2}$ can reach are $\theta_{2 \max }=1.64 \mathrm{rad}$ and $\theta_{2 \min }=-1.7$ $\operatorname{rad}(+/$ - representing opposite directions of movement of the robot arms). The minimum and 
maximum values of the nonlinearities $z_{1}$ and $z_{2}$ over the range of $\theta_{2}$ is calculated. With these minimum and maximum values of the nonlinearities, we have the membership functions

$$
\begin{aligned}
w_{11}\left(z_{1}\right) & =\frac{z_{1 \max }-z_{1}}{z_{1 \max }-z_{1 \min }} \\
w_{12}\left(z_{1}\right) & =\frac{z_{1}-z_{1 \min }}{z_{1 \max }-z_{1 \min }} \\
w_{21}\left(z_{1}\right) & =\frac{z_{2 \max }-z_{2}}{z_{2 \max }-z_{2 \min }} \\
w_{22}\left(z_{1}\right) & =\frac{z_{2}-z_{2 \min }}{z_{2 \max }-z_{2 \min }}
\end{aligned}
$$

Associating membership functions $w_{11}, w_{12}, w_{21}$, and $w_{22}$ with fuzzy sets $N_{1}^{1}, N_{2}^{1}, N_{1}^{2}$, and $N_{2}^{2}$ respectively, we have the following 4 rules representing the fuzzy model. Note that this model is equivalent to the simplified model (3-13).

$R_{1}$ : If $z_{1}$ is $N_{1}^{1}$ and $z_{2}$ is $N_{1}^{2}$ then

$$
\dot{\boldsymbol{x}}=\left[\begin{array}{cccc}
-63.7743 & 12.8930 & 0 & 0 \\
65.3550 & -629.2416 & 0 & 0 \\
1 & 0 & 0 & 0 \\
0 & 1 & 0 & 0
\end{array}\right] \boldsymbol{x}+\left[\begin{array}{cc}
344.9372 & -81.1678 \\
-353.4866 & 3961.4 \\
0 & 0 \\
0 & 0
\end{array}\right] \boldsymbol{u}
$$

$R_{2}:$ If $z_{1}$ is $N_{1}^{1}$ and $z_{2}$ is $N_{2}^{2}$ then

$$
\dot{\boldsymbol{x}}=\left[\begin{array}{cccc}
-63.7743 & 12.5410 & 0 & 0 \\
63.5707 & -628.5376 & 0 & 0 \\
1 & 0 & 0 & 0 \\
0 & 1 & 0 & 0
\end{array}\right] \boldsymbol{x}+\left[\begin{array}{cc}
344.9372 & -78.9518 \\
-343.8357 & 3956.9 \\
0 & 0 \\
0 & 0
\end{array}\right] \boldsymbol{u}
$$

$R_{3}:$ If $z_{1}$ is $N_{2}^{1}$ and $z_{2}$ is $N_{1}^{2}$ then

$$
\dot{\boldsymbol{x}}=\left[\begin{array}{cccc}
-63.7735 & 12.8929 & 0 & 0 \\
65.3542 & -629.2337 & 0 & 0 \\
1 & 0 & 0 & 0 \\
0 & 1 & 0 & 0
\end{array}\right] \boldsymbol{x}+\left[\begin{array}{cc}
344.9329 & -81.1669 \\
-353.4823 & 3961.3 \\
0 & 0 \\
0 & 0
\end{array}\right] \boldsymbol{u}
$$

$R_{4}:$ If $z_{1}$ is $N_{2}^{1}$ and $z_{2}$ is $N_{2}^{2}$ then

$$
\dot{\boldsymbol{x}}=\left[\begin{array}{cccc}
-63.7735 & 12.5409 & 0 & 0 \\
63.5699 & -628.5297 & 0 & 0 \\
1 & 0 & 0 & 0 \\
0 & 1 & 0 & 0
\end{array}\right] \boldsymbol{x}+\left[\begin{array}{cc}
344.9329 & -78.9508 \\
-343.8314 & 3956.9 \\
0 & 0 \\
0 & 0
\end{array}\right] \boldsymbol{u}
$$

where $N_{3}^{1}=N_{1}^{1}, N_{3}^{2}=N_{2}^{2}, N_{4}^{1}=N_{2}^{1}$, and $N_{4}^{2}=N_{2}^{2}$. The output matrix for all the 4 rules is the identity matrix i.e., all states are available as outputs. 
With the fuzzy model above, the simulation results are shown in Figure 3-5 and Figure 3-6, respectively, for both the links in the robot arm. These figures compare the measured output, the simulation results from the complete nonlinear system and the fuzzy model (and hence the simplified nonlinear model). It is clear from the figures that the fuzzy model almost exactly
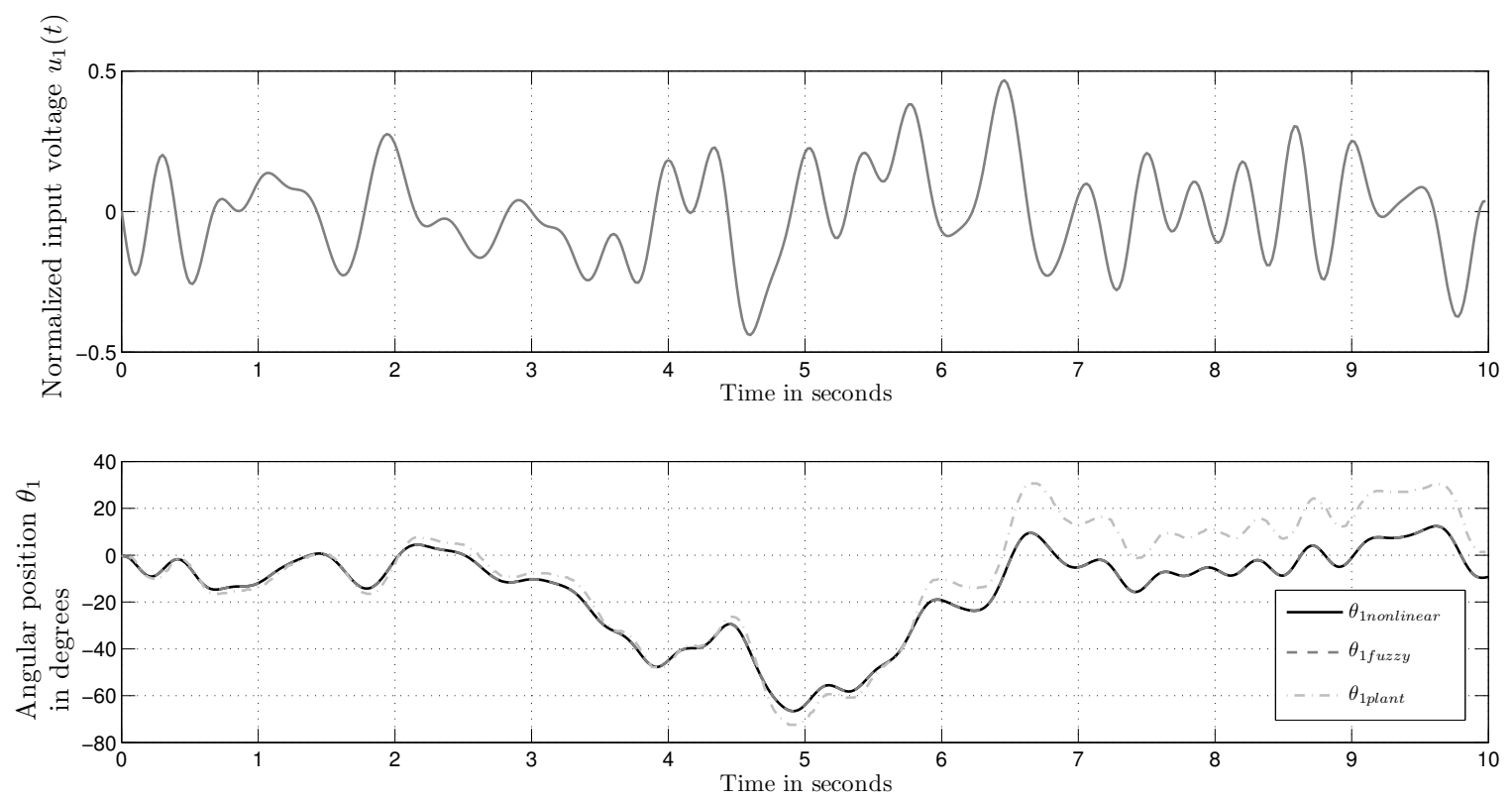

Figure 3-5: Comparison of results - measured output and simulated outputs from complete nonlinear model and TS fuzzy model - link 1.

represents the complete nonlinear model identified in the previous section, thus indicating that the better the identification of the nonlinear model, the better will be the approximation of the nonlinear system by the fuzzy model.

In this chapter, modelling of a 2-DOF robot arm has been presented. First, we estimated the parameters of the linear models of the DC motors in the robot arm joints and then nonlinear optimization has been performed to find the parameters in the nonlinear model. Until this level of modelling, no simplification has been considered. However, to construct a TS fuzzy model with a smaller number of rules, we simplify the nonlinear model by neglecting Coriolis and centrifugal forces. For the simplified nonlinear model of the 2-DOF robot arm, a TS fuzzy model that exactly represents the corresponding nonlinear model has been constructed. It has been observed that the fuzzy model almost exactly represents the complete nonlinear model. The fuzzy model constructed in this chapter will be used in the rest of the chapters. 

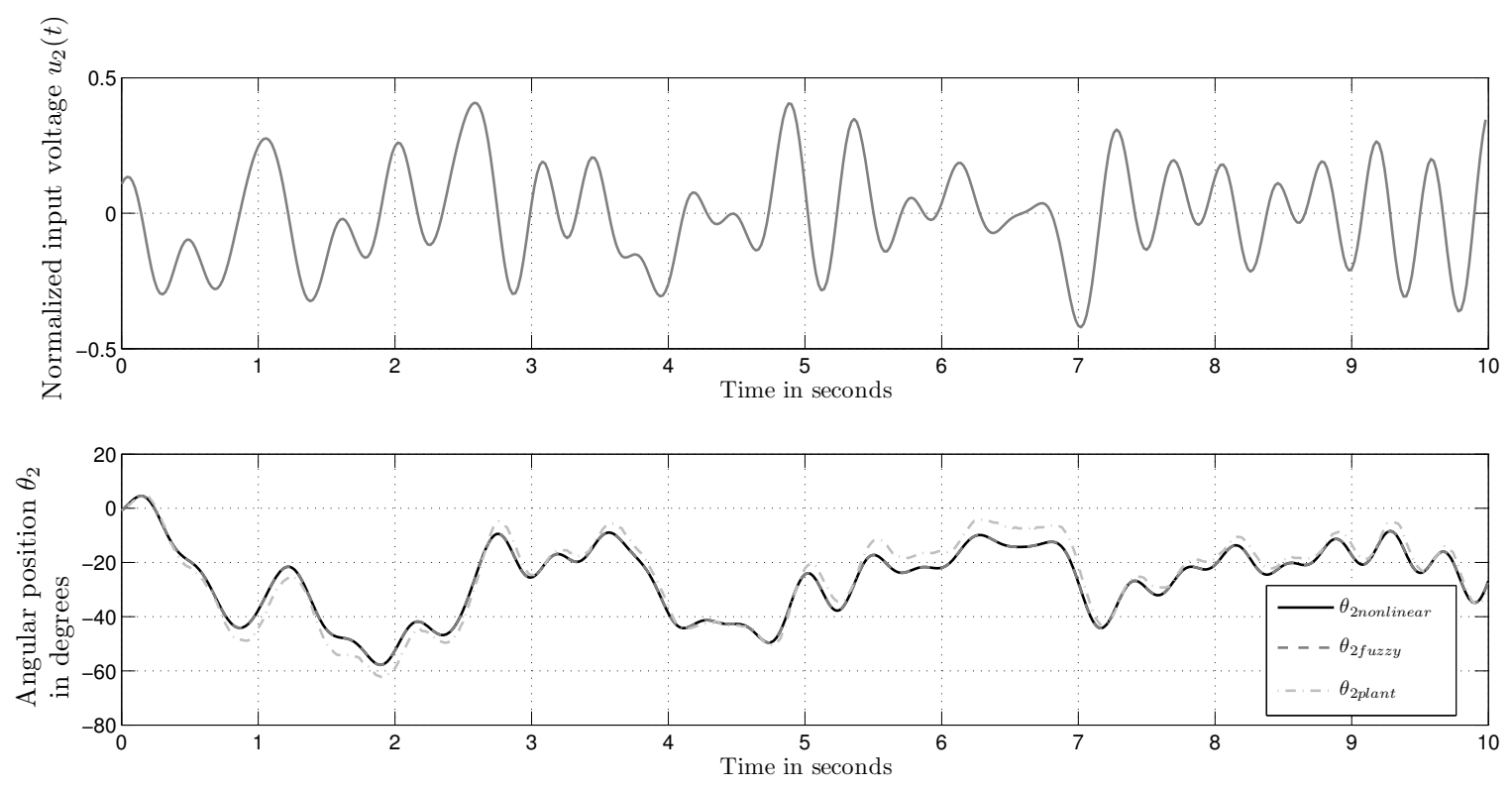

Figure 3-6: Comparison of results - measured output and simulated outputs from complete nonlinear model and TS fuzzy model - link 2. 


\section{Chapter 4}

\section{Robust fuzzy state feedback controller design}

In this chapter, we consider robust state feedback stabilizing controller design for the 2-DOF robot arm. First, the theoretical aspects of the controller design are presented. We consider uncertainty distribution structure of the form $\Delta A_{i}=M_{i} A_{\delta i}$ in the local linear models of the Takagi-Sugeno (TS) system. In the existing literature [5], uncertainty distribution structure of the form $\Delta A_{i}=D_{a i} \Delta_{a i} E_{a i}$ have been considered. Similar to [5], the design method in this chapter enables the selection of a robust controller that maximizes the norms of the uncertainty blocks $A_{\delta i}$. Uncertainties in the knowledge of parameters of the system/unmodelled dynamics can be accommodated by the uncertainty blocks in the robust controller design. Stability is guaranteed in the presence of uncertainties by the designed set of robust controllers as long as the norms of the uncertainty blocks are within predefined bounds. Performance specifications in the form of decay rate and limitation of the control input are also discussed for the robust controller design. As a preamble to the design developed for uncertain TS fuzzy systems, the first section in this chapter will present the necessary background in fuzzy state feedback stabilizing controller design. Section 4-5 presents the implementation results of the robust controller for the robot arm. The chapter ends with a section on conclusions.

\section{4-1 Fuzzy state feedback controller}

A brief introduction to fuzzy regulators has been given in Chapter 2. Conditions to guarantee stabilization of TS fuzzy systems by these regulators are available in the form of linear matrix inequalities (LMIs) [7, 19]. These results are presented in the following.

Consider a system represented by the fuzzy model

$$
\begin{aligned}
\dot{\boldsymbol{x}} & =\sum_{i=1}^{r} h_{i}(\boldsymbol{z})\left(A_{i} \boldsymbol{x}+B_{i} \boldsymbol{u}\right) \\
\boldsymbol{y} & =\sum_{i=1}^{r} h_{i}(\boldsymbol{z})\left(C_{i} \boldsymbol{x}\right)
\end{aligned}
$$


A Parallel Distributed Compensator (PDC) for this system generates a control input

$$
\boldsymbol{u}=-\sum_{i=1}^{r} h_{i}(\boldsymbol{z}) F_{i} \boldsymbol{x}
$$

which results in the closed loop dynamics

$$
\dot{\boldsymbol{x}}=\sum_{i=1}^{r} \sum_{j=1}^{r} h_{i}(\boldsymbol{z}) h_{j}(\boldsymbol{z})\left(A_{i}-B_{i} F_{j}\right) \boldsymbol{x}
$$

The closed-loop TS fuzzy model with the input (4-2) can be written as

$$
\dot{\boldsymbol{x}}=\sum_{i=1}^{r} h_{i}(\boldsymbol{z}) G_{i i} \boldsymbol{x}+2 \sum_{i<j}^{r} h_{i}(\boldsymbol{z}) h_{j}(\boldsymbol{z})\left(\frac{G_{i j}+G_{j i}}{2}\right) \boldsymbol{x}
$$

where

$G_{i j}=A_{i}-B_{i} F_{j}, \quad i, j=1,2, \ldots, r$

A theorem that presents conditions to guarantee stabilization of the closed-loop system given by (4-4) is available in [7] and given as follows.

Theorem 3. [Y] The presence of a common positive definite matrix $P$ satisfying the conditions

$$
\begin{aligned}
& G_{i i}^{T} P+P G_{i i}<0 \\
& \frac{\left(G_{i j}+G_{j i}\right)^{T}}{2} P+P \frac{\left(G_{i j}+G_{j i}\right)}{2} \leq 0 \quad \text { for } i<j
\end{aligned}
$$

for $i, j=1,2, \ldots, r$ except the pairs $(i, j)$ such that $h_{i}(\boldsymbol{z}) h_{j}(\boldsymbol{z})=0, \forall t$ confirms the stability of the fuzzy control system given by (4-4).

When the number of rules in a system becomes very large, it becomes difficult to find a common positive definite matrix $P$. Hence, relaxed stability conditions have been proposed in [7] and [19].

Theorem 4. [7, 19] The number of rules $s$ that fire at a given time $t$, is less than or equal to $r$ (that is $1<s \leq r$ ). The system (4-4) is asymptotically stable in the large if there exist a common positive definite matrix $P$ and a common positive semidefinite matrix $Q$ such that

$$
\begin{aligned}
& G_{i i}^{T} P+P G_{i i}+(s-1) Q<0 \\
& \frac{\left(G_{i j}+G_{j i}\right)^{T}}{2} P+P \frac{\left(G_{i j}+G_{j i}\right)}{2}-Q \leq 0 \quad \text { for } i<j
\end{aligned}
$$

for $i, j=1,2, \ldots, r$ except the pairs $(i, j)$ such that $h_{i}(\boldsymbol{z}) h_{j}(\boldsymbol{z})=0, \forall t$.

This theorem is relaxed in the sense that the conditions that are derived accommodate the possibility that all rules in a TS fuzzy model need not be active simultaneously. In case of TS fuzzy models derived by the sector nonlinearity approach, all the rules will be active at any instant in time. However, even in this case (when all rules are active), it is shown in [5] that the conditions in Theorem 4 increase the space of state and input matrices over which valid $P$ and $Q$ matrices can be found. 
Control performances have also been considered in [7, 19]. A theorem to accommodate decay rate has been given in [7]. The convergence rate of a system is at least $\alpha$ if $\dot{V}(\boldsymbol{x}(t)) \leq$ $-2 \alpha V(\boldsymbol{x}(t))$ [21], where $\alpha$ is the decay rate. Results for stabilization with decay rate $\alpha$ is given below.

Theorem 5. [7] The condition that $\dot{V}(\boldsymbol{x}(t)) \leq-2 \alpha V(\boldsymbol{x}(t))$ for the Lyapunov function $V(\boldsymbol{x}(t))=\boldsymbol{x}^{T}$ P $\boldsymbol{x}$ for all trajectories of the system is equivalent to

$$
\begin{aligned}
& G_{i i}^{T} P+P G_{i i}+(s-1) Q+2 \alpha P<0 \\
& \frac{\left(G_{i j}+G_{j i}\right)^{T}}{2} P+P \frac{\left(G_{i j}+G_{j i}\right)}{2}-Q+2 \alpha P \leq 0 \quad \text { for } i<j
\end{aligned}
$$

for $i, j=1,2, \ldots, r$ except the pairs $(i, j)$ such that $h_{i}(\boldsymbol{z}) h_{j}(\boldsymbol{z})=0, \forall t$ and $s>1$ and $\alpha>0$.

A generalized eigenvalue minimization problem formulation to obtain the largest lower bound on the decay rate $\alpha$ is suggested in [19].

Further performance constraints like control input constraint and output constraints are considered in [19]. Given that the initial conditions $\boldsymbol{x}(0)$ are known such that $\|\boldsymbol{x}(0)\| \leq \phi$, LMI conditions for $\|\boldsymbol{u}(t)\| \leq \zeta$ to be true for all $t \geq 0$ are given in [19]. Here, $\zeta$ is the control input norm bound and $\phi$ is the initial condition norm bound and are discussed further in Section 4-4.

In the following section, we consider an uncertain TS fuzzy model and design a robust controller for it. Literature contains robust controller design for uncertainty in the state matrices represented as $\Delta A_{i}=D_{a i} \Delta_{a i} E_{a i}[5]$. In this thesis we use the adaptive observer concept available in literature [1] to obtain estimates of uncertainty in the system and to reuse the uncertainty estimates from the adaptive observer to design a controller. This will require both the adaptive observer and the controller to use the same uncertainty distribution structure to represent the uncertainty in the state matrices. Since the uncertainty distribution structure in the adaptive observer is of the form $\Delta A_{i}=M_{i} A_{\delta i}{ }^{2}$ a robust controller design for uncertainty distribution structure $\Delta A_{i}=M_{i} A_{\delta i}$ is presented in the following.

\section{4-2 Uncertain TS fuzzy model}

The TS fuzzy model considered is as follows.

$$
\begin{aligned}
& R_{i}: \text { If } z_{1} \text { is } N_{i}^{1} \text { and } z_{2} \text { is } N_{i}^{2}, \ldots \text { and } z_{p} \text { is } N_{i}^{p} \text {, then } \\
& \qquad \begin{aligned}
\dot{\boldsymbol{x}} & =A_{i} \boldsymbol{x}+B_{i} \boldsymbol{u}+M_{i} A_{\delta i} \boldsymbol{x} \\
\boldsymbol{y} & =C_{i} \boldsymbol{x}
\end{aligned}
\end{aligned}
$$

for $i=1,2, \ldots, r$.

The different variables in the above equation are the same as in Chapter 2. The $M_{i} A_{\delta i}$ is

\footnotetext{
${ }^{2} \Delta A_{i}$ is the uncertainty in the matrices $A_{i}, M_{i}$ are the uncertainty distribution matrices, $A_{\delta i}$ are the uncertainty blocks, and $M_{i} A_{\delta i}$ and $D_{a i} \Delta_{a i} E_{a i}$ are two different uncertainty distribution structures for the uncertainty $\Delta A_{i}$
} 
referred to as the uncertainty distribution structure since it distributes the uncertainty in $A_{\delta i}$ by using $M_{i}$ into the positions of $\Delta A_{i}$ where uncertainty is expected to exist.

The model (4-8) can be rewritten as

$$
\begin{aligned}
\dot{\boldsymbol{x}} & =\sum_{i=1}^{r} h_{i}(\boldsymbol{z})\left(A_{i} \boldsymbol{x}+B_{i} \boldsymbol{u}+M_{i} A_{\delta i} \boldsymbol{x}\right) \\
\boldsymbol{y} & =C_{i} \boldsymbol{x}
\end{aligned}
$$

where $h_{i}$ are the normalized membership functions.

The uncertainty in the above fuzzy model exists in the consequents of the rules, i.e., in the state matrices of the local linear models. The following section discusses the robust controller design for the model as represented in equation (4-9) with the uncertainty blocks bounded by a known norm i.e., $\left\|A_{\delta i}\right\| \leq \mu_{i}, i=1,2, \ldots, r$.

\section{4-3 Robust stability conditions with norm-bounded uncertainty}

This section presents the conditions that guarantee closed-loop stability when a PDC controller is used to stabilize (4-9). The control input generated by a PDC is

$$
\boldsymbol{u}=-\sum_{j=1}^{r} h_{j}(\boldsymbol{z}) F_{j} \boldsymbol{x}
$$

This results in the following closed-loop system dynamics

$$
\begin{aligned}
\dot{\boldsymbol{x}}= & \sum_{i=1}^{r} \sum_{j=1}^{r} h_{i}(\boldsymbol{z}) h_{j}(\boldsymbol{z})\left(A_{i}-B_{i} F_{j}+M_{i} A_{\delta i}\right) \boldsymbol{x} \\
= & \sum_{i=1}^{r} h_{i}{ }^{2}(\boldsymbol{z})\left(A_{i}-B_{i} F_{i}+M_{i} A_{\delta i}\right) \boldsymbol{x} \\
& +\sum_{i=1}^{r} \sum_{i<j} h_{i}(\boldsymbol{z}) h_{j}(\boldsymbol{z})\left(A_{i}-B_{i} F_{j}+A_{j}-B_{j} F_{i}+M_{i} A_{\delta i}+M_{j} A_{\delta j}\right) \boldsymbol{x}
\end{aligned}
$$

Considering a common quadratic Lyapunov function

$$
V=\boldsymbol{x}^{T} P \boldsymbol{x}
$$

we can state the conditions to be satisfied to guarantee stability of the uncertain TS system (4-9) in the form of the following theorem.

Theorem 6. The uncertain fuzzy system (4-9) with $\left\|A_{\delta i}\right\| \leq \mu_{i}, i=1,2, \ldots, r$ where $\mu_{i}$ are known constants, is stabilized by the PDC (4-10) if there exist a common positive definite matrix $P\left(X=P^{-1}\right)$ and matrices $N_{i}, i=1,2, \ldots, r$ that satisfy

$$
\begin{aligned}
& X>0, \\
& S_{i i}<0, \\
& T_{i j}<0, \quad i, j=1,2, \ldots, r \text { and } i<j \text { s.t } h_{i} h_{j} \neq 0
\end{aligned}
$$


where $S_{i i}$ and $T_{i j}$ are given by the equations

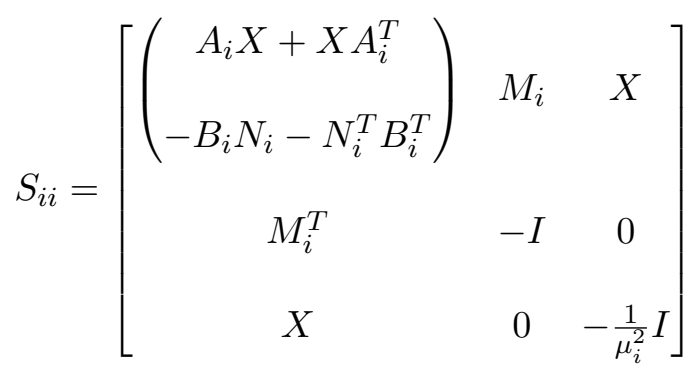

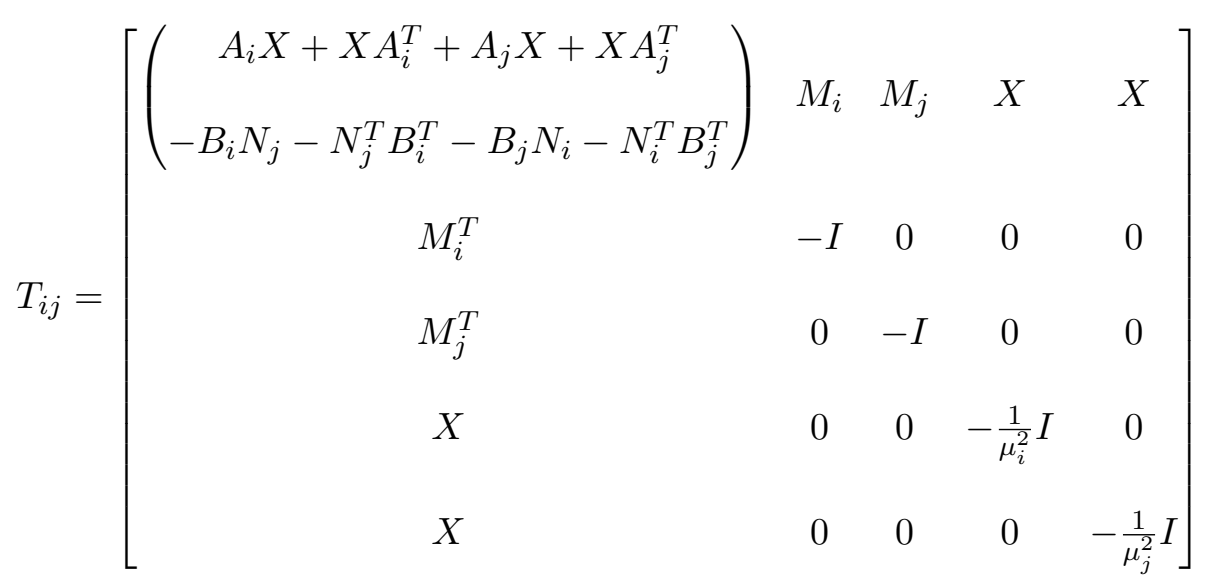

Proof. With the Lyapunov function

$$
V=\boldsymbol{x}^{T} P \boldsymbol{x}
$$

we have

$$
\begin{aligned}
\dot{V}= & \boldsymbol{x}^{T} P \dot{\boldsymbol{x}}+\dot{\boldsymbol{x}}^{T} P \boldsymbol{x} \\
= & \sum_{i=1}^{r} h_{i}{ }^{2}(\boldsymbol{z}) \boldsymbol{x}^{T}\left[P\left(A_{i}-B_{i} F_{i}+M_{i} A_{\delta i}\right)+\left(A_{i}-B_{i} F_{i}+M_{i} A_{\delta i}\right)^{T} P\right] \boldsymbol{x} \\
& \quad+\sum_{i=1}^{r} \sum_{i<j} h_{i}(\boldsymbol{z}) h_{j}(\boldsymbol{z}) \boldsymbol{x}^{T}\left[P\left(A_{i}-B_{i} F_{j}+M_{i} A_{\delta i}\right)+\left(A_{i}-B_{i} F_{j}+M_{i} A_{\delta i}\right)^{T} P\right. \\
& \left.+P\left(A_{j}-B_{j} F_{i}+M_{j} A_{\delta j}\right)+\left(A_{j}-B_{j} F_{i}+M_{j} A_{\delta j}\right)^{T} P\right] \boldsymbol{x}
\end{aligned}
$$




$$
\begin{aligned}
& =\sum_{i=1}^{r} h_{i}{ }^{2}(\boldsymbol{z}) \boldsymbol{x}^{T}\left[P\left(A_{i}-B_{i} F_{i}\right)+\left(A_{i}-B_{i} F_{i}\right)^{T} P+P\left(M_{i} A_{\delta i}\right)+\left(M_{i} A_{\delta i}\right)^{T} P\right] \boldsymbol{x} \\
& +\sum_{i=1}^{r} \sum_{i<j} h_{i}(\boldsymbol{z}) h_{j}(\boldsymbol{z}) \boldsymbol{x}^{T}\left[P\left(A_{i}-B_{i} F_{j}\right)+\left(A_{i}-B_{i} F_{j}\right)^{T} P+P\left(M_{i} A_{\delta i}\right)+\left(M_{i} A_{\delta i}\right)^{T} P\right. \\
& \left.+P\left(A_{j}-B_{j} F_{i}\right)+\left(A_{j}-B_{j} F_{i}\right)^{T} P+P\left(M_{j} A_{\delta j}\right)+\left(M_{j} A_{\delta j}\right)^{T} P\right] \boldsymbol{x} \\
& =\sum_{i=1}^{r} h_{i}{ }^{2}(\boldsymbol{z}) \boldsymbol{x}^{T}\left[P\left(A_{i}-B_{i} F_{i}\right)+\left(A_{i}-B_{i} F_{i}\right)^{T} P+P\left(M_{i} A_{\delta i}\right)+\left(M_{i} A_{\delta i}\right)^{T} P\right. \\
& \left.+P M_{i} M_{i}^{T} P-P M_{i} M_{i}^{T} P+A_{\delta i}^{T} A_{\delta i}-A_{\delta i}^{T} A_{\delta i}\right] \boldsymbol{x} \\
& +\sum_{i=1}^{r} \sum_{i<j} h_{i}(\boldsymbol{z}) h_{j}(\boldsymbol{z}) \boldsymbol{x}^{T}\left[P\left(A_{i}-B_{i} F_{j}\right)+\left(A_{i}-B_{i} F_{j}\right)^{T} P+P\left(M_{i} A_{\delta i}\right)+\left(M_{i} A_{\delta i}\right)^{T} P\right. \\
& +P\left(A_{j}-B_{j} F_{i}\right)+\left(A_{j}-B_{j} F_{i}\right)^{T} P+P\left(M_{j} A_{\delta j}\right)+\left(M_{j} A_{\delta j}\right)^{T} P+P M_{i} M_{i}^{T} P \\
& \left.-P M_{i} M_{i}^{T} P+A_{\delta i}^{T} A_{\delta i}-A_{\delta i}^{T} A_{\delta i}+P M_{j} M_{j}^{T} P-P M_{j} M_{j}^{T} P+A_{\delta j}^{T} A_{\delta j}-A_{\delta j}^{T} A_{\delta j}\right] \boldsymbol{x} \\
& =\sum_{i=1}^{r} h_{i}{ }^{2}(\boldsymbol{z}) \boldsymbol{x}^{T}\left[P\left(A_{i}-B_{i} F_{i}\right)+\left(A_{i}-B_{i} F_{i}\right)^{T} P+P M_{i} M_{i}^{T} P+A_{\delta i}^{T} A_{\delta i}\right. \\
& \left.-P M_{i}\left(M_{i}^{T} P-A_{\delta i}\right)+A_{\delta i}^{T}\left(M_{i}^{T} P-A_{\delta i}\right)\right] \boldsymbol{x} \\
& +\sum_{i=1}^{r} \sum_{i<j} h_{i}(\boldsymbol{z}) h_{j}(\boldsymbol{z}) \boldsymbol{x}^{T}\left[P\left(A_{i}-B_{i} F_{j}\right)+\left(A_{i}-B_{i} F_{j}\right)^{T} P+P\left(A_{j}-B_{j} F_{i}\right)\right. \\
& +\left(A_{j}-B_{j} F_{i}\right)^{T} P+P M_{i} M_{i}^{T} P+P M_{j} M_{j}^{T} P+A_{\delta i}^{T} A_{\delta i}+A_{\delta j}^{T} A_{\delta j}-P M_{i}\left(M_{i}^{T} P-A_{\delta i}\right) \\
& \left.+A_{\delta i}^{T}\left(M_{i}^{T} P-A_{\delta i}\right)-P M_{j}\left(M_{j}^{T} P-A_{\delta j}\right)+A_{\delta j}^{T}\left(M_{j}^{T} P-A_{\delta j}\right)\right] \boldsymbol{x} \\
& =\sum_{i=1}^{r} h_{i}{ }^{2}(\boldsymbol{z}) \boldsymbol{x}^{T}\left[P\left(A_{i}-B_{i} F_{i}\right)+\left(A_{i}-B_{i} F_{i}\right)^{T} P+P M_{i} M_{i}^{T} P+A_{\delta i}^{T} A_{\delta i}\right. \\
& \left.-\left(M_{i}^{T} P-A_{\delta i}\right)^{T}\left(M_{i}^{T} P-A_{\delta i}\right)\right] \boldsymbol{x} \\
& +\sum_{i=1}^{r} \sum_{i<j} h_{i}(\boldsymbol{z}) h_{j}(\boldsymbol{z}) \boldsymbol{x}^{T}\left[P\left(A_{i}-B_{i} F_{j}\right)+\left(A_{i}-B_{i} F_{j}\right)^{T} P+P\left(A_{j}-B_{j} F_{i}\right)\right. \\
& +\left(A_{j}-B_{j} F_{i}\right)^{T} P+P M_{i} M_{i}^{T} P+P M_{j} M_{j}^{T} P+A_{\delta i}^{T} A_{\delta i}+A_{\delta j}^{T} A_{\delta j} \\
& \left.-\left(M_{i}^{T} P-A_{\delta i}\right)^{T}\left(M_{i}^{T} P-A_{\delta i}\right)-\left(M_{j}^{T} P-A_{\delta j}\right)^{T}\left(M_{j}^{T} P-A_{\delta j}\right)\right] \boldsymbol{x}
\end{aligned}
$$

If

$$
\begin{aligned}
& P\left(A_{i}-B_{i} F_{i}\right)+\left(A_{i}-B_{i} F_{i}\right)^{T} P+P M_{i} M_{i}^{T} P+\mu_{i}^{2} I<0 \\
& P\left(A_{i}-B_{i} F_{j}\right)+\left(A_{i}-B_{i} F_{j}\right)^{T} P+P\left(A_{j}-B_{j} F_{i}\right)+\left(A_{j}-B_{j} F_{i}\right)^{T} P \\
& +P M_{i} M_{i}^{T} P+P M_{j} M_{j}^{T} P+\mu_{i}{ }^{2} I+\mu_{j}{ }^{2} I<0
\end{aligned}
$$

hold for $i, j=1,2, \ldots, r$ and $i<j$, then we have $\dot{V}<0$. 
Left and right multiplying the inequalities in (4-15) by $P^{-1}$ and introducing a new variable $X=P^{-1}$, we can write

$$
\begin{aligned}
& \left(A_{i}-B_{i} F_{i}\right) X+X\left(A_{i}-B_{i} F_{i}\right)^{T}+M_{i} M_{i}^{T}+X \mu_{i}^{2} X<0 \\
& \left(A_{i}-B_{i} F_{j}\right) X+X\left(A_{i}-B_{i} F_{j}\right)^{T}+\left(A_{j}-B_{j} F_{i}\right) X+X\left(A_{j}-B_{j} F_{i}\right)^{T} \\
& +M_{i} M_{i}^{T}+M_{j} M_{j}^{T}+X \mu_{i}^{2} X+X \mu_{j}^{2} X<0
\end{aligned}
$$

$i, j=1,2, \ldots, r$ and $i<j$.

With the change of variables $N_{i}=F_{i} X$ and hence, $F_{i}=N_{i} P$, we can write the inequalities in (4-16) as

$$
\begin{aligned}
& A_{i} X-B_{i} N_{i}+X A_{i}^{T}-N_{i}^{T} B_{i}+M_{i} M_{i}^{T}+X \mu_{i}^{2} X<0 \\
& \left.A_{i} X-B_{i} N_{j}+X A_{i}^{T}-N_{j}^{T} B_{i}\right)^{T}+A_{j} X-B_{j} N_{i}+X A_{j}^{T}-N_{i}^{T} B_{j} \\
& +M_{i} M_{i}^{T}+M_{j} M_{j}^{T}+X \mu_{i}^{2} X+X \mu_{j}^{2} X<0
\end{aligned}
$$

$i, j=1,2, \ldots, r$ and $i<j$.

Converting the conditions in (4-17) to linear matrix inequalities using Schur complements, we have

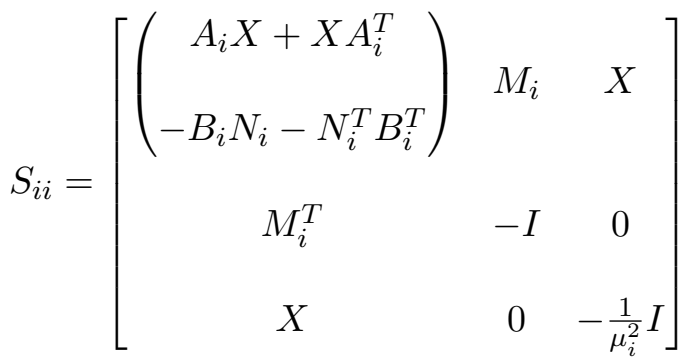

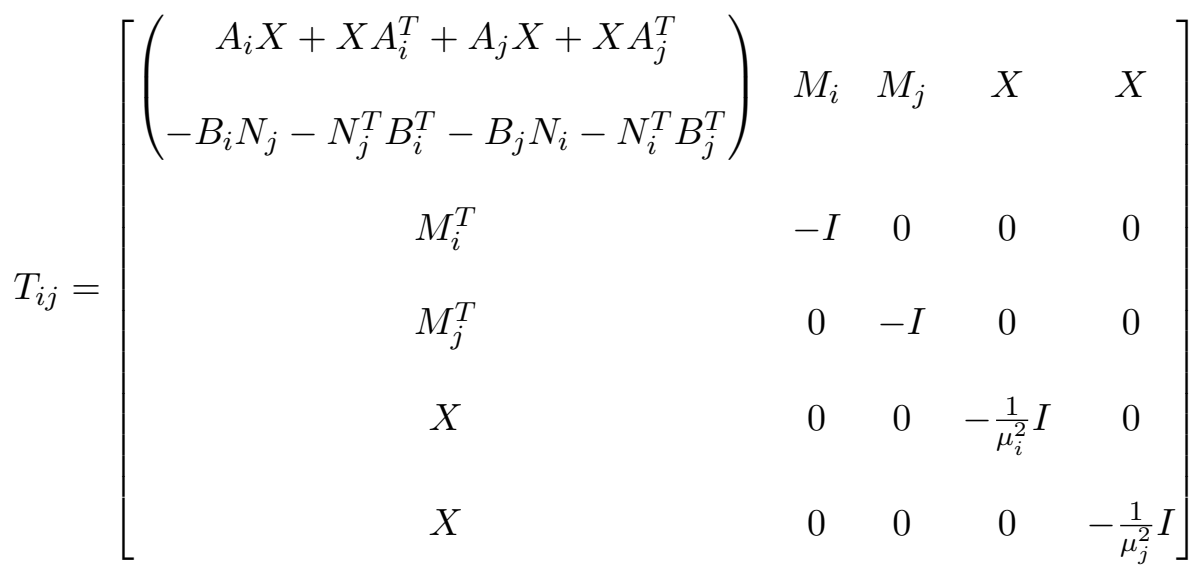

and

$$
\begin{aligned}
& S_{i i}<0 \quad i, j=1,2, \ldots, r \text { and } i<j \\
& T_{i j}<0 \quad i, j=1,2, \ldots, r \text { and } i<j
\end{aligned}
$$


The above LMIs guarantee that $\dot{V}<0$ since we have

$$
\begin{aligned}
A_{\delta i}{ }^{T} A_{\delta i} & <\mu_{i}{ }^{2} I \\
-\left(M_{i}^{T} P-A_{\delta i}\right)^{T}\left(M_{i}^{T} P-A_{\delta i}\right) & <0 \\
-\left(M_{i}^{T} P-A_{\delta i}\right)^{T}\left(M_{i}^{T} P-A_{\delta i}\right)-\left(M_{j}^{T} P-A_{\delta j}\right)^{T}\left(M_{j}^{T} P-A_{\delta j}\right) & <0
\end{aligned}
$$

In the theorem above, it is considered that an upper bound on the uncertainty block norms are known. Given the system state matrices, the norms of the uncertainty blocks for which a controller can be found can be maximized as part of designing the robust controller [5]. This is done by maximizing the linear combination of the squares of the norms of the uncertainty blocks along with satisfying the linear matrix inequality (LMI)s in Theorem 6 .

Theorem 7. The uncertain fuzzy system (4-9) is stabilized by the PDC (4-10) if there exist a common positive definite matrix $P\left(X=P^{-1}\right)$ and matrices $N_{i}, i=1,2, \ldots, r$ that satisfy

$$
\begin{aligned}
& \underset{\mu_{i}^{2}, X, N_{1}, N_{2} \ldots N_{r}}{\operatorname{maximize}} \quad \sum_{i=1}^{r} \beta_{i} \mu_{i}{ }^{2} \\
& \text { subject to } \\
& X>0, \\
& \quad S_{i i}<0, \\
& T_{i j}<0, \quad i, j=1,2, \ldots, r \text { and } i<j \text { s.t } h_{i} h_{j} \neq 0
\end{aligned}
$$

where $\beta_{i}$ are design parameters and $S_{i i}$ and $T_{i j}$ are given by equations (4-18) and (4-19).

Proof. This theorem follows from Theorem 6 by maximizing the norms of the uncertainty blocks.

Based on relaxed stability conditions [5], the above theorems can be extended to accommodate the possibility that all rules in a TS fuzzy model need not be active simultaneously. Thus, we have the following two theorems.

Theorem 8. The uncertain fuzzy system (4-9) with $\left\|A_{\delta i}\right\| \leq \mu_{i}, i=1,2, \ldots, r$ where $\mu_{i}$ are known constants, is stabilized by the PDC (4-10) if there exist a common positive definite matrix $P\left(X=P^{-1}\right)$, a positive semidefinite matrix $Q_{0}$ and matrices $N_{i}, i=1,2, \ldots, r$ that satisfy

$$
\begin{aligned}
& X>0, \\
& S_{i i}+(s-1) Y_{1}<0, \\
& T_{i j}-2 Y_{2}<0, \quad i, j=1,2, \ldots, r \text { and } i<j \text { s.t } h_{i} h_{j} \neq 0
\end{aligned}
$$

where

$Y_{1}=\operatorname{bkdiag}^{3}\left(\begin{array}{lll}Y_{0} & 0 & 0\end{array}\right)$,

$Y_{2}=\operatorname{bkdiag}\left(\begin{array}{lllll}Y_{0} & 0 & 0 & 0 & 0\end{array}\right)$,

$Y_{0}=X Q_{0} X$,

$s$ is the number of rules active for all time $t(1<s \leq r)$ and $S_{i i}$ and $T_{i j}$ are given by equations (4-18) and (4-19).

\footnotetext{
${ }^{3}$ bkdiag stands for block diagonal.
} 
Proof. See Appendix C.

Theorem 9. The uncertain fuzzy system (4-9) is stabilized by the PDC (4-10) if there exist a common positive definite matrix $P\left(X=P^{-1}\right)$, a positive semidefinite matrix $Q_{0}$ and matrices $N_{i}, i=1,2, \ldots, r$ that satisfy

$$
\begin{aligned}
& \underset{\mu_{i}{ }^{2}, X, N_{1}, N_{2} \ldots N_{r}}{\operatorname{maximize}} \sum_{i=1}^{r} \beta_{i} \mu_{i}^{2} \\
& \text { subject to } \\
& \quad X>0 \\
& \quad S_{i i}+(s-1) Y_{1}<0, \\
& \quad T_{i j}-2 Y_{2}<0, \quad i, j=1,2, \ldots, r \text { and } i<j \text { s.t } h_{i} h_{j} \neq 0
\end{aligned}
$$

where

$Y_{1}=\operatorname{bkdiag}\left(\begin{array}{lll}Y_{0} & 0 & 0\end{array}\right)$,

$Y_{2}=\operatorname{bkdiag}\left(\begin{array}{lllll}Y_{0} & 0 & 0 & 0 & 0\end{array}\right)$,

$Y_{0}=X Q_{0} X$,

$s$ is the number of rules active for all time $t(1<s \leq r), \beta_{i}$ are the design parameters and $S_{i i}$ and $T_{i j}$ are given by equations (4-18) and (4-19).

Proof. This theorem follows from Theorem 8 by maximizing the norms of the uncertainty blocks.

Given a TS system with state matrices $A_{i}$, uncertainties $\Delta A_{i}=M_{i} A_{\delta i}$, and $\left\|A_{\delta i}\right\| \leq \mu_{i}$, Theorems 8 and 9 provide conditions for stabilization by a PDC with relaxed stability conditions. For the complete derivation of Theorem 8 see Appendix C. Theorems 6 and 8 in this section present robust controller design without and with relaxed stability conditions. Theorems 7 and 9 maximize the norms of the uncertainty blocks as part of the robust controller design. In the following section, we discuss the addition of performance specifications to the robust controller design, namely decay rate and control input constraint given an upper bound on the initial state norm.

\section{4-4 Performance specifications for the controller}

Along with stabilizing a system, the speed of the system response is very important. A decay rate specification can be added to the controller design for this purpose. In practice, since actuators will be able to provide signals that are limited in their value, a specification that can limit the control input generated by the controller is useful. We discuss both these performance specifications for the robust stabilizing controller in the following.

\section{4-4-1 Decay rate specification}

The response of the stabilizing controller can be made faster by introducing a decay rate $(\alpha)$ specification in the stability conditions. Introducing the condition $\dot{V}(\boldsymbol{x}(t)) \leq-2 \alpha V(\boldsymbol{x}(t))$ in the LMIs of Theorems 6, 7, 8, and 9 we have the following. 
Theorem 10. The uncertain fuzzy system (4-9) with $\left\|A_{\delta i}\right\| \leq \mu_{i}, i=1,2, \ldots, r$ where $\mu_{i}$ are known constants, is stabilized by the PDC (4-10) and the closed-loop system has a decay rate of at least $\alpha$ if there exist a common positive definite matrix $P\left(X=P^{-1}\right)$ and matrices $N_{i}$, $i=1,2, \ldots, r$ that satisfy

$$
\begin{aligned}
& X>0, \\
& \widehat{S}_{i i}<0, \\
& \widehat{T}_{i j}<0, \quad i, j=1,2, \ldots, r \text { and } i<j \text { s.t } h_{i} h_{j} \neq 0
\end{aligned}
$$

where

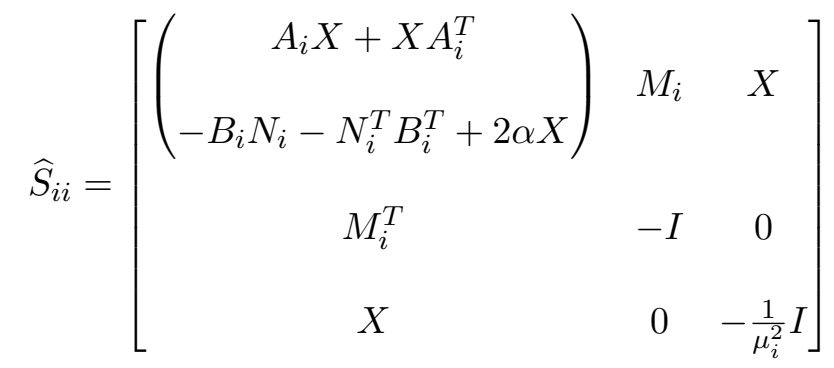

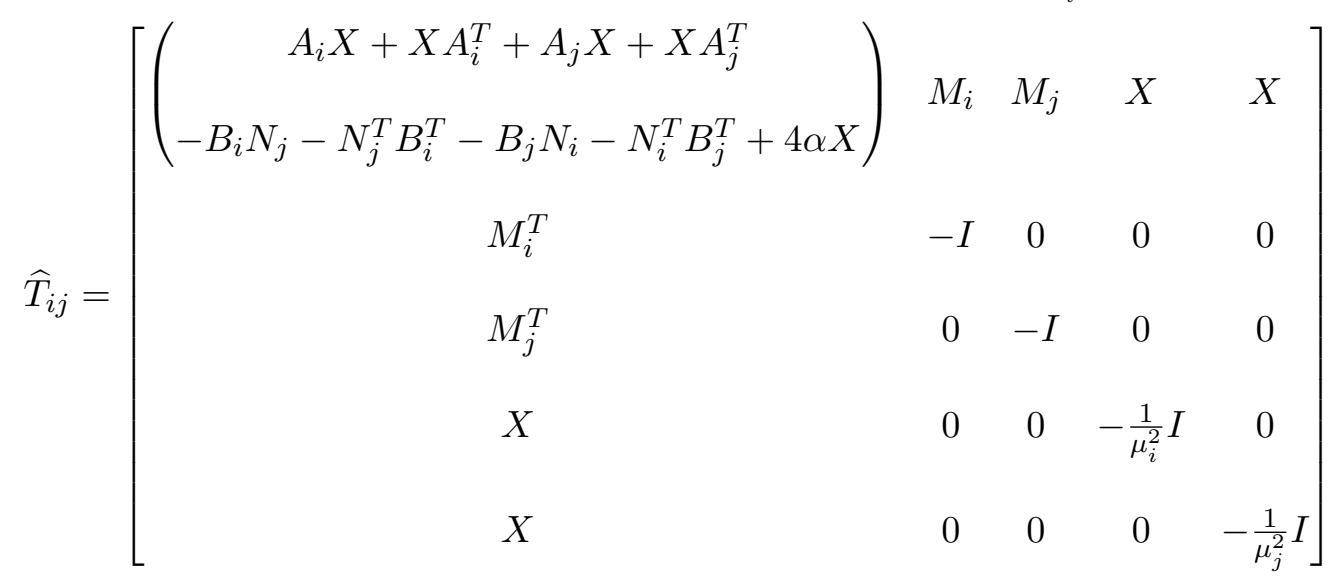

Proof. See Appendix C.

In the above theorem, the bounds on the uncertainty block norms are assumed to be known. The following theorem is an extension of Theorem 10 to obtain the maximum bounds on the norms of the uncertainty blocks for a desired specification of $\alpha$.

Theorem 11. The uncertain fuzzy system (4-9) is stabilized by the PDC (4-10) and the closed-loop system has a decay rate of at least $\alpha$ if there exist a common positive definite matrix $P\left(X=P^{-1}\right)$ and $N_{i}, i=1,2, \ldots, r$ that satisfy

$$
\begin{aligned}
& \underset{\mu_{i}^{2}, X, N_{1}, N_{2} \ldots N_{r}}{\operatorname{maximize}} \quad \sum_{i=1}^{r} \beta_{i} \mu_{i}{ }^{2} \\
& \text { subject to } \\
& X>0, \\
& \widehat{S}_{i i}<0, \\
& \widehat{T}_{i j}<0, \quad i, j=1,2, \ldots, r \text { and } i<j \text { s.t } h_{i} h_{j} \neq 0
\end{aligned}
$$


where $\alpha$ is the specified decay rate, $\beta_{i}, i=1,2, \ldots, r$ are the design parameters and $\widehat{S}_{i i}$ and $\widehat{T}_{i j}, i, j=1,2, \ldots, r$ and $i<j$ are given by equations (4-22) and (4-23)

Proof. The proof follows from Theorem 10 by maximizing the norms of the uncertainty blocks.

The above two theorems provided conditions that guarantee stability of the closed-loop system with a decay rate of atleast $\alpha$. Theorem 10 provided conditions when bounds on the uncertainty block norms are known and Theorem 11 provided conditions such that the maximum bounds on the uncertainty block norms can be found as part of the controller design process. These two theorems can be extended to include the relaxed stability conditions leading to the following two theorems.

Theorem 12. The uncertain fuzzy system (4-9) is stabilized by the PDC (4-10) and the closed-loop system has decay rate of at least $\alpha$ if there exist a common positive definite matrix $P\left(X=P^{-1}\right)$, a positive semidefinite matrix $Q_{0}$, and matrices $N_{i}, i=1,2, \ldots, r$ that satisfy

$$
\begin{aligned}
& X>0, \\
& \widehat{S}_{i i}+(s-1) Y_{1}<0, \\
& \widehat{T}_{i j}-2 Y_{2}<0, \quad i, j=1,2, \ldots, r \text { and } i<j \text { s.t } h_{i} h_{j} \neq 0
\end{aligned}
$$

where

$Y_{1}=\operatorname{bkdiag}\left(\begin{array}{lll}Y_{0} & 0 & 0\end{array}\right)$,

$Y_{2}=\operatorname{bkdiag}\left(\begin{array}{lllll}Y_{0} & 0 & 0 & 0 & 0\end{array}\right)$,

$Y_{0}=X Q_{0} X$,

$s$ is the number of rules active for all time $t(1<s \leq r), \alpha$ is the specified decay rate and $\widehat{S}_{i i}$ and $\widehat{T}_{i j}, i=1,2, \ldots, r, i<j$ are given by equations (4-22) and (4-23).

Proof. The proof follows from Theorems 8 and 10 .

In the above theorem, maximizing the norms of the uncertainty blocks will provide the conditions that guarantee stability of the closed-loop system with a decay rate of at least $\alpha$ and the maximum uncertainty norm bounds for which stability is guaranteed are obtained as part of the design process. The result of this exercise is presented in the following theorem.

Theorem 13. The uncertain fuzzy system (4-9) is stabilized by the PDC (4-10) and the closed-loop system has decay rate of at least $\alpha$ if there exist a common positive definite matrix $P\left(X=P^{-1}\right)$, a positive semidefinite matrix $Q_{0}$, and matrices $N_{i}, i=1,2, \ldots, r$ that satisfy

$$
\begin{aligned}
& \underset{\mu_{i}^{2}, X, N_{1}, N_{2} \ldots N_{r}}{\operatorname{maximize}} \sum_{i=1}^{r} \beta_{i} \mu_{i}{ }^{2} \\
& \text { subject to } \\
& \quad X>0 \\
& \quad \widehat{S}_{i i}+(s-1) Y_{1}<0, \\
& \quad \widehat{T}_{i j}-2 Y_{2}<0, \quad i, j=1,2, \ldots, r \text { and } i<j \text { s.t } h_{i} h_{j} \neq 0
\end{aligned}
$$


where

$Y_{1}=\operatorname{bkdiag}\left(\begin{array}{lll}Y_{0} & 0 & 0\end{array}\right)$,

$Y_{2}=\operatorname{bkdiag}\left(\begin{array}{lllll}Y_{0} & 0 & 0 & 0 & 0\end{array}\right)$,

$Y_{0}=X Q_{0} X$,

$s$ is the number of rules active for all time $t(1<s \leq r), \alpha$ is the specified decay rate, $\beta_{i}, i=1,2, \ldots, r$ are the design parameters and $\widehat{S}_{i i}$ and $\widehat{T}_{i j}, i=1,2, \ldots, r, i<j$ are given by equations (4-22) and (4-23).

Proof. The proof follows from Theorem 12 by maximizing the norms of the uncertainty blocks.

The above four theorems presented the conditions to be satisfied for the PDC given by equation (4-10) to stabilize the uncertain fuzzy system (4-9) with a decay rate of at least $\alpha$. In the following, additional conditions to be satisfied along with the conditions in any of the above eight theorems to stabilize (4-9) with a constraint on the input is presented.

\section{4-4-2 Constraint on control input}

LMIs corresponding to a constraint on the control input given an upper bound on the initial state norm, can be added to the robust controller design as in [5]. For the purpose of continuity, the LMIs corresponding to the control input constraint are reproduced in the form of a theorem. The conditions in this theorem can be added to the conditions from the above theorems to guarantee stabilization along with constraint on the control input.

Theorem 14. [5] For the system (4-9) stabilized by (4-10) and given an upper bound $\phi$ on the initial state (i.e., $\|\boldsymbol{x}(0)\| \leq \phi)$, the constraint on the control input $\|\boldsymbol{u}(t)\| \leq \zeta$ is satisfied for all $t>0$, if the following LMIs are satisfied

$$
\begin{gathered}
{\left[\begin{array}{cc}
X & N_{i}^{T} \\
N_{i} & \zeta^{2} I
\end{array}\right] \geq 0 \quad \text { for } i=1,2, \ldots, r} \\
X \geq \phi^{2} I
\end{gathered}
$$

Both decay rate and control input constraint can be specified for a robust controller. Hence, it is possible to combine the conditions in Theorem 14 with the conditions in Theorems 6-13.

In the following section, the design of the stabilizing controller is demonstrated on the simplified 2-DOF robot arm and simulation results are presented.

\section{4-5 Controller design for 2-DOF robot arm}

The 2-DOF robot arm fuzzy model has been constructed in Chapter 3. The TS fuzzy model presented in Section 3-5 of Chapter 3 consists of 4 rules. Uncertainty is assumed to be in the state matrices. An uncertainty in any of the parameters $P_{1}, P_{2}, P_{3}, b_{1}$ or $b_{2}$ can 
essentially result in uncertainty in the positions $(1,1),(1,2),(2,1)$ or $(2,2)$ of the state matrices (3-35)-(3-38) based on the presence of the above mentioned parameters in those positions. A constant uncertainty is assumed to exist in the position $(1,2)$ of the state matrices which can be attributed to an uncertainty in the parameter $b_{2}$. As a simplification, the constant uncertainty value is considered to be the same in all the rules. The simulations can as well be performed with uncertainty in any position of the state matrices.

The controller in this section is designed using Theorem 11 and Theorem $14^{4}$. In the design of the robust controller, the following assumptions are considered.

- Since the TS fuzzy model has been constructed using the sector nonlinearity approach, the number of active rules at all time $t>0$ is 4 (i.e. $s=r=4$ ).

- A constant uncertainty is considered in position $(1,2)$ of the state matrices in all the rules i.e.,

$$
\Delta A_{i}=\left[\begin{array}{cccc}
0 & c_{12} & 0 & 0 \\
0 & 0 & 0 & 0 \\
0 & 0 & 0 & 0 \\
0 & 0 & 0 & 0
\end{array}\right]
$$

where $c_{12}$ is the non-zero value.

- Sensor information is assumed to be exactly the same as the state information. In practice, appropriate filtering is needed if sensor noise is present.

The specifications considered are as follows.

- The decay rate is $\alpha=1$.

- The limit on the control input is $\|\boldsymbol{u}(t)\| \leq 1$.

In the following, the evolution of states and control input is presented for two different initial conditions.

\section{Case 1}

For this case, assume that $\|\boldsymbol{x}(0)\| \leq 1$. The initial state is taken as

$\boldsymbol{x}(0)_{\text {deg }}=\left[\begin{array}{llll}28.6479 & 40.1070 & -9.7403 & 14.8969\end{array}\right]^{T}$ i.e., $\boldsymbol{x}(0)_{\text {rad }}=\left[\begin{array}{llll}0.5 & -0.7 & -0.17 & 0.26\end{array}\right]^{T}$ and hence $\|\boldsymbol{x}(0)\|=0.9146^{5}$. With the aforementioned assumptions for the system and the

\footnotetext{
${ }^{4}$ The system of LMIs is solved using 'mincx' function of MATLAB's LMI toolbox.

${ }^{5}$ The norm calculations for the initial state considers the states to be measured in radians and radians $/ s$. For convenience, the state values are presented in degrees and degrees/s as well.
} 


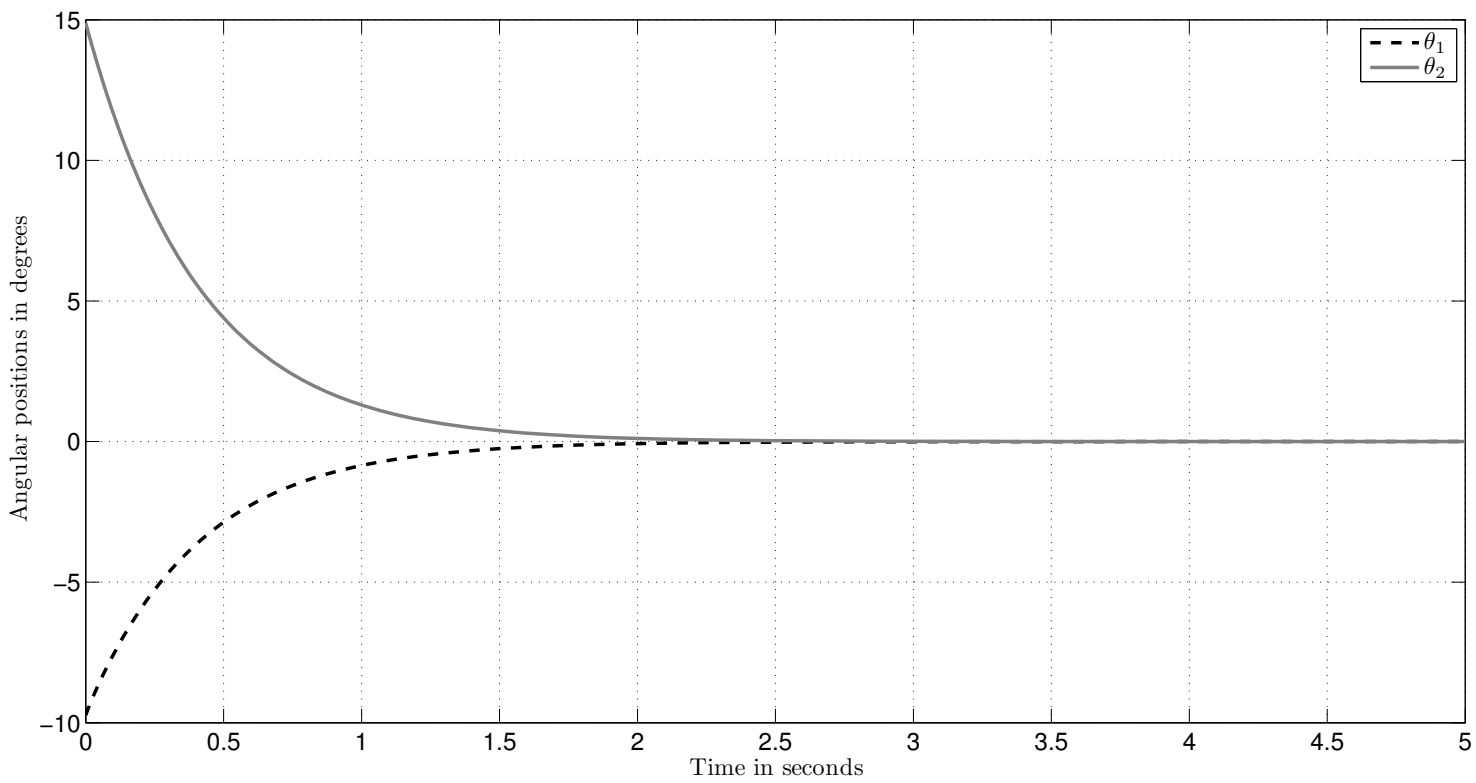

Figure 4-1: Evolution of positions with controller designed for $\|\boldsymbol{x}(0)\| \leq 1$ and initial condition $\boldsymbol{x}(0)_{\operatorname{deg}}=\left[\begin{array}{llll}28.6479 & 40.1070 & -9.7403 & 14.8969\end{array}\right]^{T}$.

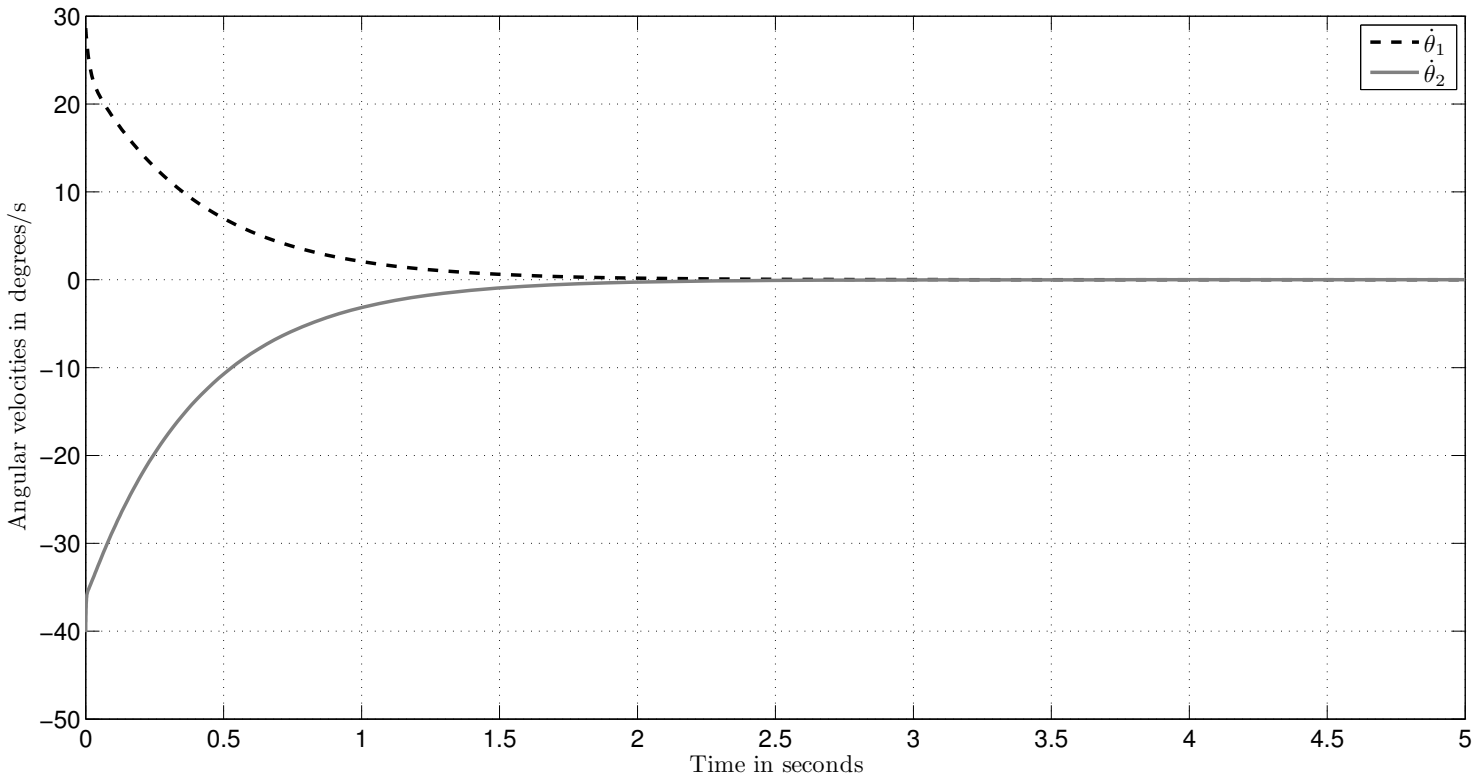

Figure 4-2: Evolution of velocities with controller designed for $\|\boldsymbol{x}(0)\| \leq 1$ and initial condition $\boldsymbol{x}(0)_{\operatorname{deg}}=\left[\begin{array}{llll}28.6479 & 40.1070 & -9.7403 & 14.8969\end{array}\right]^{T}$.

specifications for the controller, the values of the controller gains obtained are as follows. 


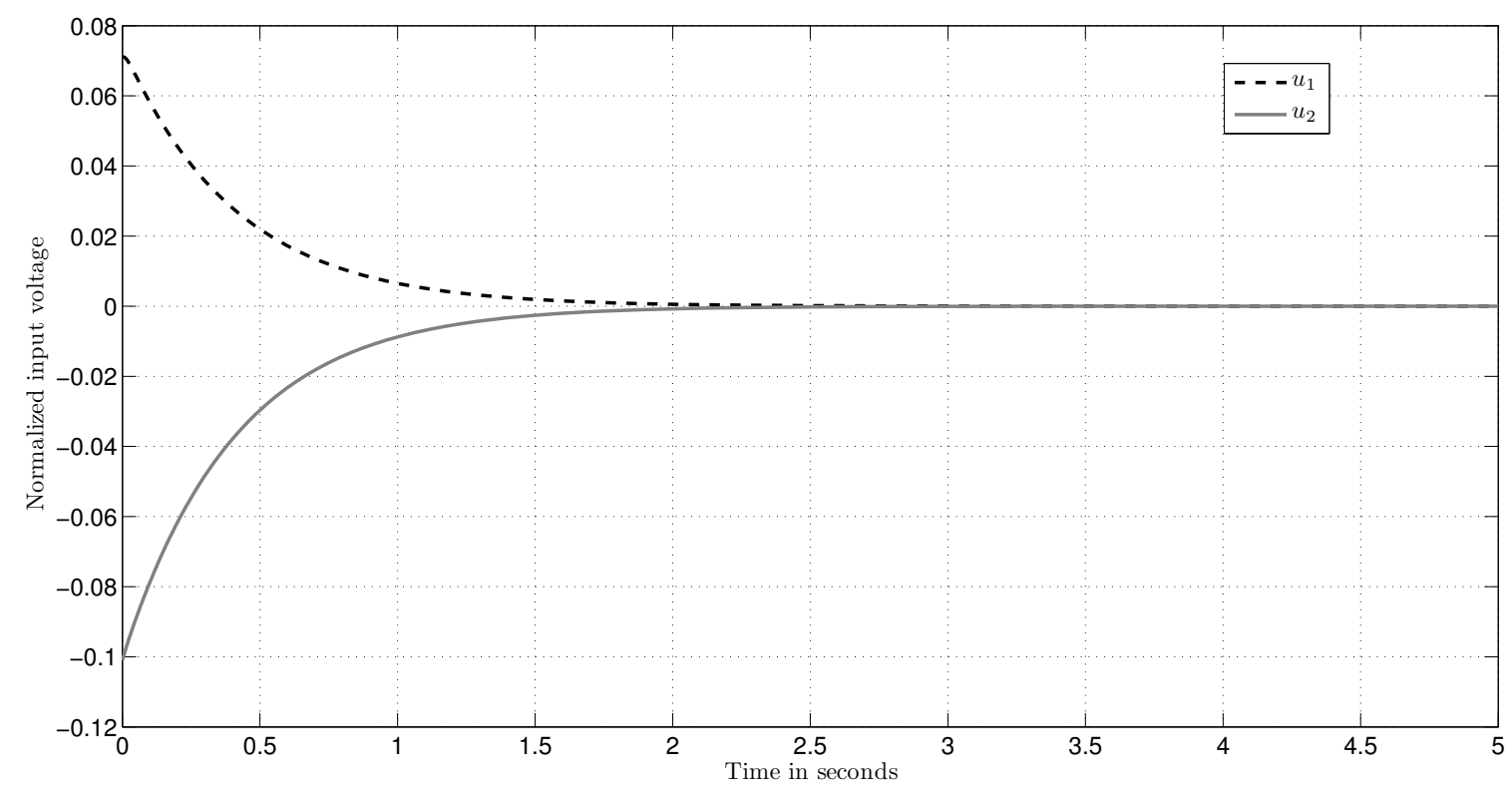

Figure 4-3: Control input with controller designed for $\|\boldsymbol{x}(0)\| \leq 1$ and initial condition $\boldsymbol{x}(0)_{\operatorname{deg}}=\left[\begin{array}{llll}28.6479 & 40.1070 & -9.7403 & 14.8969\end{array}\right]^{T}$.

$F_{1}=\left[\begin{array}{llll}0.0432 & 0.0009 & 0.5434 & 0.0016 \\ 0.0077 & 0.0042 & 0.0184 & 0.3967\end{array}\right]$
$F_{2}=\left[\begin{array}{llll}0.0432 & 0.0009 & 0.5433 & 0.0016 \\ 0.0075 & 0.0042 & 0.0178 & 0.3967\end{array}\right]$
$F_{3}=\left[\begin{array}{llll}0.0432 & 0.0009 & 0.5434 & 0.0016 \\ 0.0077 & 0.0042 & 0.0184 & 0.3967\end{array}\right]$
$F_{4}=\left[\begin{array}{llll}0.0432 & 0.0009 & 0.5433 & 0.0016 \\ 0.0075 & 0.0042 & 0.0178 & 0.3967\end{array}\right]$

and the maximum bounds on the uncertainty norms are: $\mu_{1}=0.637796, \mu_{2}=0.637796$, $\mu_{3}=0.637796$ and $\mu_{4}=0.637796$.

With a constant uncertainty of 0.6 (since norm of the uncertainty will be thus smaller than $\mu_{i}$ given by the controller design) at location $(1,2)$ in the state matrices, the evolution of the states and the control inputs is given in Figure 4-1, Figure 4-2 and Figure 4-3.

\section{Case 2}

For this case, the controller is designed with initial conditions $\|\boldsymbol{x}(0)\| \leq 2$. To simulate a trajectory, the initial state is taken to be $\boldsymbol{x}(0)_{d e g}=\left[\begin{array}{llll}28.6479 & -40.1070 & -9.7403 & 44.6907\end{array}\right]^{T}$ i.e., $\boldsymbol{x}(0)_{\text {rad }}=\left[\begin{array}{llll}0.5 & -0.7 & -0.17 & 0.78\end{array}\right]^{T}$ and hence $\|\boldsymbol{x}(0)\|=1.1736$

With $\|\boldsymbol{x}(0)\| \leq 2$, the controller gains obtained are as follows. 


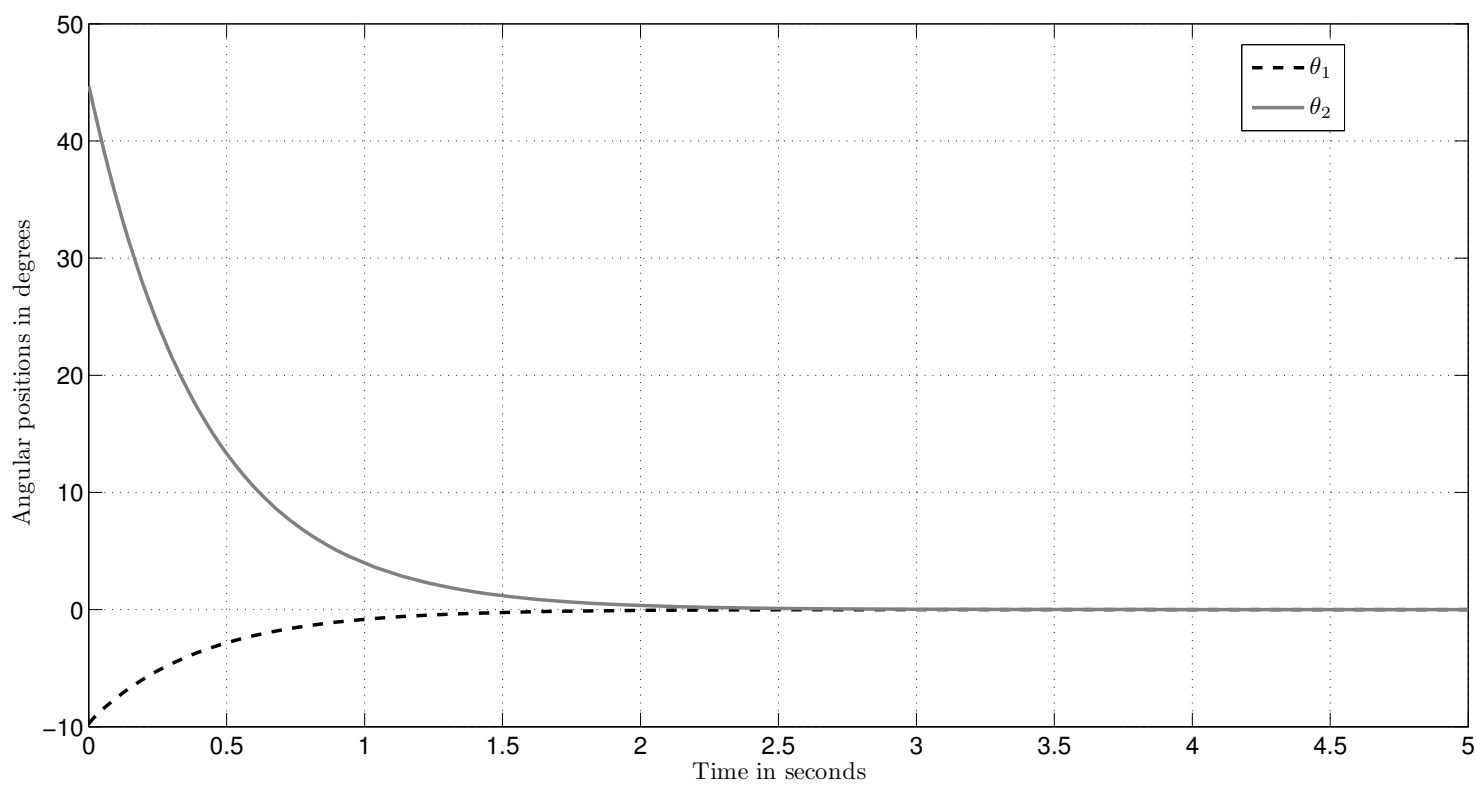

Figure 4-4: Evolution of positions with controller designed for $\|\boldsymbol{x}(0)\| \leq 2$ and initial condition $\boldsymbol{x}(0)_{\text {deg }}=\left[\begin{array}{llll}28.6479 & -40.1070 & -9.7403 & 44.6907\end{array}\right]^{T}$.

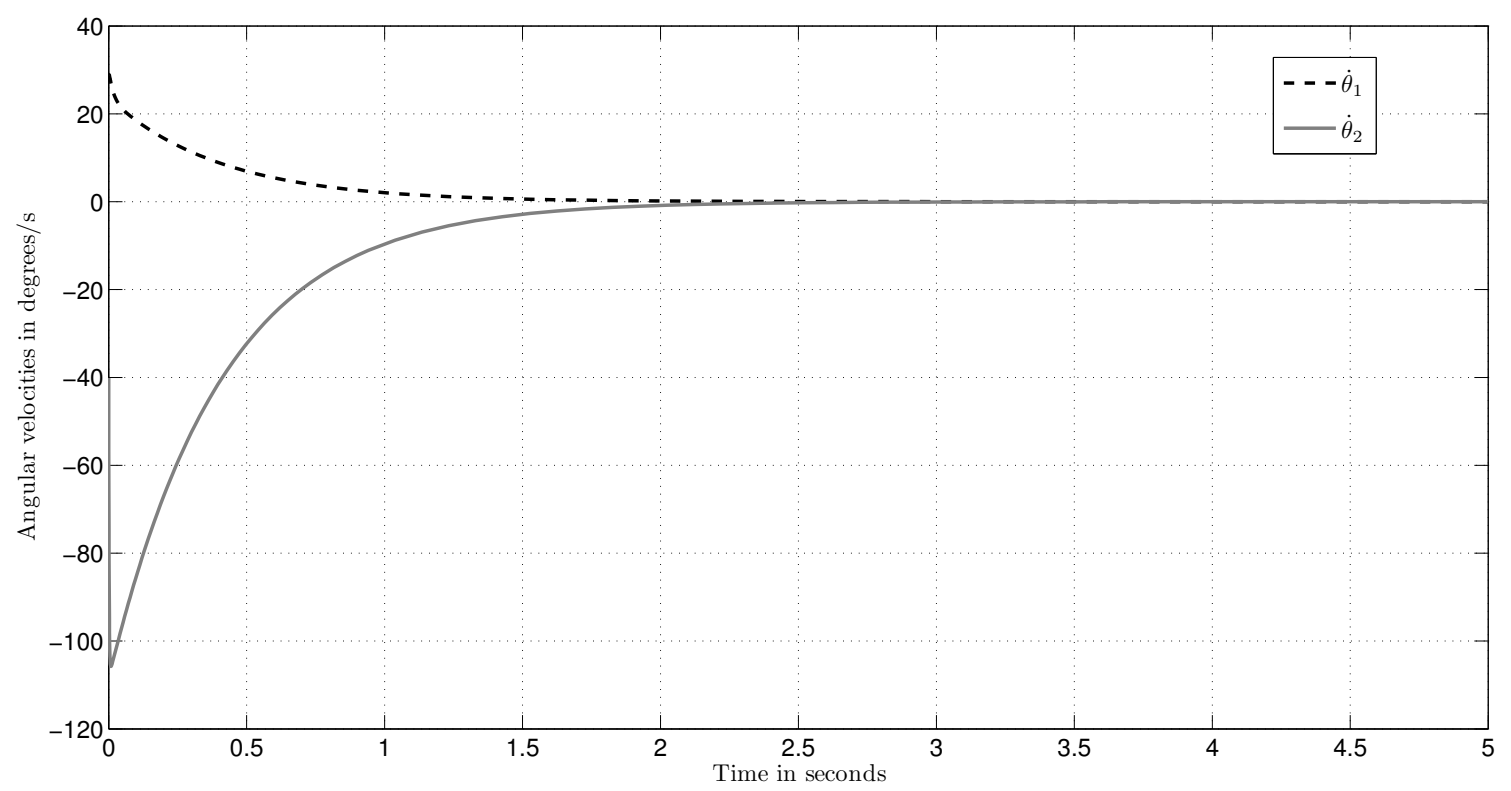

Figure 4-5: Evolution of velocities with controller designed for $\|\boldsymbol{x}(0)\| \leq 2$ and initial condition $\boldsymbol{x}(0)_{\text {deg }}=\left[\begin{array}{llll}28.6479 & -40.1070 & -9.7403 & 44.6907\end{array}\right]^{T}$.

$F_{1}=\left[\begin{array}{cccc}0.0102 & 0.0002 & 0.4619 & -0.0014 \\ 0.0034 & 0.0011 & 0.0078 & 0.3867\end{array}\right]$ 


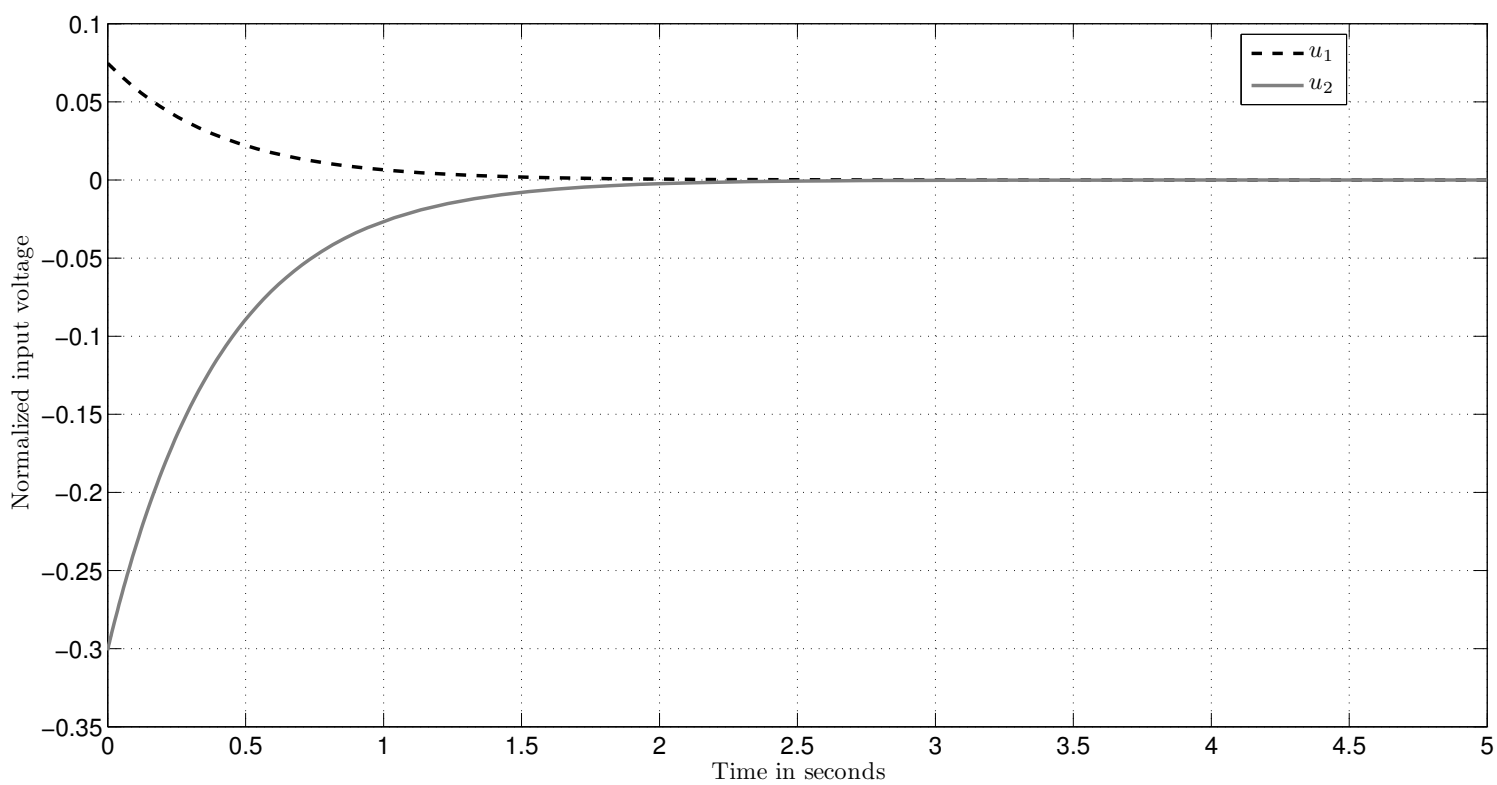

Figure 4-6: Control input with controller designed for $\|\boldsymbol{x}(0)\| \leq 2$ and initial condition $\boldsymbol{x}(0)_{\text {deg }}=\left[\begin{array}{llll}28.6479 & -40.1070 & -9.7403 & 44.6907\end{array}\right]^{T}$.

$F_{2}=\left[\begin{array}{lllc}0.0102 & 0.0002 & 0.4618 & -0.0014 \\ 0.0032 & 0.0011 & 0.0075 & 0.3867\end{array}\right]$
$F_{3}=\left[\begin{array}{lllc}0.0102 & 0.0002 & 0.4619 & -0.0014 \\ 0.0034 & 0.0011 & 0.0078 & 0.3867\end{array}\right]$
$F_{4}=\left[\begin{array}{lllc}0.0102 & 0.0002 & 0.4618 & -0.0014 \\ 0.0032 & 0.0011 & 0.0075 & 0.3867\end{array}\right]$

and the maximum bounds on the uncertainty norms are: $\mu_{1}=0.318436, \mu_{2}=0.318436$, $\mu_{3}=0.318436$ and $\mu_{4}=0.318436$.

With a constant uncertainty of 0.3 (since the norm of the uncertainty will be thus smaller than $\mu_{i}$ given by the controller design) at location $(1,2)$ in the state matrices, the evolution of the states and the control input is given in Figure 4-4, Figure 4-5 and Figure 4-6.

\section{4-6 Conclusions}

This chapter discussed robust controller design. The uncertainty distribution structure in the local linear models of the TS fuzzy model is of the form $\Delta A_{i}=M_{i} A_{\delta i}$. The uncertainties are bounded by known norm values. The maximum norm of the uncertainty blocks that the controller can stabilize can be obtained as part of the design process. Other specifications such as decay rate and control input constraint can be added to the controller design. The decay rate is specified by the user. As an extension of the work done in this chapter, further work can be done to simultaneously maximize the decay rate and the norms of the uncertainty blocks. 
The type of uncertainty that can be stabilized by the controller designed in this chapter is not limited to constant uncertainties in the state matrices. The controller will also be able to stabilize uncertainties of the form $\Delta A_{i}=M_{i} A_{\delta i}$ that are unmodelled nonlinearities or time varying as long as their norm is smaller than the maximum uncertainty bound obtained from robust controller design.

The uncertainty distribution structure considered in this thesis differs from that considered in [5] in the sense that $\Delta A_{i}=M_{i} A_{\delta i}$ is a simpler uncertainty pattern compared to $\Delta A_{i}=$ $D_{a i} \Delta_{a i} E_{a i}$. Moreover, $\Delta A_{i}=M_{i} A_{\delta i}$ lacks the flexibility to concentrate uncertainty in a desired subsection of the state matrix.

In the following chapter, we discuss the applicability of an adaptive observer to the robot arm. 


\section{Chapter 5}

\section{Adaptive observer}

The previous chapter presented the robust controller design for the uncertainty distribution structure $\Delta A_{i}=M_{i} A_{\delta i}$. In this chapter, we present the design of an adaptive observer to estimate constant uncertainties in the state matrices of the Takagi-Sugeno (TS) fuzzy model of the robot arm. The adaptive observer design guarantees asymptotic stability given an upper bound $\mu_{\max }$ on the uncertainty block norm $\left\|A_{\delta i}\right\|$. The value of $\mu_{\max }$ is thus a very important design consideration. The theory of adaptive observer is presented in the first section of this chapter. This is followed by the results of applying the adaptive observer to the robot arm fuzzy model. The effect of varying the value of $\mu_{\max }$ is analyzed and presented in Section 5-2 of this chapter. Section 5-3 presents the analysis of uncertainty structure of the TS model in designing uncertainty estimation experiments.

\section{5-1 Introduction}

Observers are generally designed to estimate the states of a system with known information about the current output and input of the system. However, it becomes difficult to estimate the states of the system when there are uncertainties in the system. The estimation becomes further complicated in the absence of complete information about the input to the system [21]. Adaptive observers have been researched in the case of linear systems and nonlinear systems. A discussion regarding adaptive observers for TS fuzzy models is available in [21]. Based on the availability of an existing model of the plant, approaches for observer design to estimate unknown inputs and unmodelled dynamics are provided. Consider the model

$$
\begin{aligned}
\dot{\boldsymbol{x}} & =\sum_{i=1}^{r} h_{i}(\boldsymbol{z})\left(A_{i} \boldsymbol{x}+B_{i} \boldsymbol{u}+a_{i}+M_{i} \boldsymbol{d}\right) \\
\boldsymbol{y} & =\sum_{i=1}^{r} h_{i}(\boldsymbol{z})\left(C_{i} \boldsymbol{x}\right)
\end{aligned}
$$

where 
- $A_{i}, B_{i}, C_{i}, M_{i}, a_{i}$ are known matrices and biases

- $\boldsymbol{d}$ is an unknown input representing either unmodelled dynamics or disturbances

- $M_{i}$ are disturbance distribution matrices

The observer considered is of the form:

$$
\begin{aligned}
\dot{\hat{\boldsymbol{x}}} & =\sum_{i=1}^{r} h_{i}(\widehat{\boldsymbol{z}})\left(A_{i} \widehat{\boldsymbol{x}}+B_{i} \boldsymbol{u}+a_{i}+M_{i} \widehat{\boldsymbol{d}}+L_{i}(\boldsymbol{y}-\widehat{\boldsymbol{y}})\right) \\
\widehat{\boldsymbol{y}} & =\sum_{i=1}^{r} h_{i}(\widehat{\boldsymbol{z}})\left(C_{i} \widehat{\boldsymbol{x}}\right) \\
\dot{\hat{\boldsymbol{d}}} & =f(\widehat{\boldsymbol{d}}, \boldsymbol{h}(\widehat{\boldsymbol{z}}), \widehat{\boldsymbol{x}}, \boldsymbol{y})
\end{aligned}
$$

with $L_{i}, i=1,2, \ldots, r$ being the gain matrices for every rule of the observer and $f$ being the update law for $\boldsymbol{d}$.

Observer design has been considered for two cases. One is to estimate unknown polynomial inputs and the other is to estimate unmodelled dynamics. In estimating the unknown polynomial inputs, two sets of stability conditions have been derived based on whether the scheduling variables are dependent on the state variables or not. The conditions have been derived for $\boldsymbol{d}$ being a $(p-1)^{t h}$ degree polynomial in time and also another case where the $p^{t h}$ derivative of $\boldsymbol{d}$ is bounded by a known constant.

In estimating the unmodelled dynamics, a fuzzy system of the following form is considered.

$$
\dot{\boldsymbol{x}}=\sum_{i=1}^{r} h_{i}(\boldsymbol{z})\left(A_{i} \boldsymbol{x}+B_{i} \boldsymbol{u}+M_{i}\left(A_{\delta i} \boldsymbol{x}+B_{\delta i} \boldsymbol{u}+\theta_{i}\right)\right)
$$

where the matrices $A_{\delta i}, B_{\delta i}$ and vectors $\theta_{i}$ are unknown and $A_{\delta i}$ is bounded by $\mu_{\max }\left(\left\|A_{\delta i}\right\| \leq\right.$ $\left.\mu_{\max }\right)$. [21] provides sufficient conditions to determine stability and to design an asymptotically stable observer to estimate the states $\boldsymbol{x}$ and constant matrices $A_{\delta i}, B_{\delta i}$ and parameter vectors $\theta_{i}$.

In case of uncertainty only in the state matrices and a common output matrix for all rules, the fuzzy model and the corresponding adaptive observer are as follows.

For the uncertain TS fuzzy system

$$
\begin{aligned}
\dot{\boldsymbol{x}} & =\sum_{i=1}^{r} h_{i}(\boldsymbol{z})\left(A_{i} \boldsymbol{x}+B_{i} \boldsymbol{u}+M_{i}\left(A_{\delta i} \boldsymbol{x}\right)\right) \\
\boldsymbol{y} & =C \boldsymbol{x}
\end{aligned}
$$

assuming that the scheduling variables do not depend on the states to be estimated, the observer is

$$
\begin{aligned}
\dot{\widehat{\boldsymbol{x}}} & =\sum_{i=1}^{r} h_{i}(\boldsymbol{z})\left(A_{i} \boldsymbol{x}+B_{i} \boldsymbol{u}+L_{i}(\boldsymbol{y}-\widehat{\boldsymbol{y}})+M_{i}\left(\widehat{A}_{\delta i} \widehat{\boldsymbol{x}}\right)\right) \\
\widehat{\boldsymbol{y}} & =C \widehat{\boldsymbol{x}}
\end{aligned}
$$


with the update law

$$
\dot{\widehat{A}}_{\delta i}=\boldsymbol{f}_{i}\left(\widehat{A}_{\delta i}, \boldsymbol{h}(\boldsymbol{z}), \widehat{\boldsymbol{x}}, \boldsymbol{y}\right) \quad i=1,2, \ldots, r
$$

where $L_{i}, i=1,2, \ldots, r$ are the observer gain matrices for each rule and the update laws $\boldsymbol{f}_{i}, i=1,2, \ldots, r$ are determined so that the estimation errors $(\boldsymbol{x}-\widehat{\boldsymbol{x}})$ and $\left(A_{\delta i}-\widehat{A}_{\delta i}\right)$ asymptotically converge to zero. The error dynamics are given as

$$
\left.\dot{\boldsymbol{e}}=\sum_{i=1}^{r} h_{i}(\boldsymbol{z})\left[\left(A_{i}-L_{i} C+M_{i} A_{\delta i}\right) \boldsymbol{e}+M_{i}\left(\bar{A}_{\delta i}\right) \widehat{\boldsymbol{x}}\right)\right]
$$

with $\bar{A}_{\delta i}=A_{\delta i}-\widehat{A}_{\delta i}$. We also assume that the matrices $M_{i}, i=1,2, \ldots, r$ have full column rank, and $\operatorname{rank}\left(C M_{i}\right)=\operatorname{rank}\left(M_{i}\right), i=1,2, \ldots, r$. This assumption ensures that the uncertainty is observable from the available measurements.

The following theorem, rewritten for constant uncertainties only in the state matrices of the fuzzy model, provides the conditions to guarantee asymptotic stability of the adaptive observer for the uncertainty block norm $\left\|A_{\delta i}\right\| \leq \mu_{\max }$.

Theorem 15. [1] The error dynamics (5-7) are asymptotically stable, if the membership functions $h_{i}, i=1,2, \ldots, r$ are smooth, all rules are sufficiently excited and there exist matrices $P=P^{T}>0, Q=Q^{T}>0$ and $L_{i}, i=1,2, \ldots, r$ such that

$$
\begin{array}{r}
\mathcal{H}\left(P\left(A_{i}-L_{i} C\right)\right)<-Q \\
\left\|M_{i}\right\| \mu_{\max } \leq \frac{\lambda_{\min }(Q)}{\lambda_{\max }(P)}
\end{array}
$$

for $i=1,2, \ldots, r$ and with the uncertainty matrix update law

$$
\dot{\widehat{A}}_{\delta i}=h_{i}(\boldsymbol{z}) M_{i}^{T} P C^{\dagger} \boldsymbol{e}_{y} \widehat{x}^{T}
$$

where $C^{\dagger}$ is the Moore-Penrose pseudoinverse of $C$.

Proof. See [1].

The measure of the uncertainty estimation error in the adaptive observer is defined as the sum of squares of element-wise uncertainty error in all local linear models i.e.,

$$
A_{\delta e}=\sum_{i=1}^{r} \operatorname{tr}\left(\bar{A}_{\delta i}^{T} \bar{A}_{\delta i}\right)=\sum_{i=1}^{r} \sum_{j=1}^{n_{r}} \sum_{k=1}^{n_{c}} \bar{A}_{\delta i}^{2}(j, k)
$$

where $n_{c}$ is the number of columns and $n_{r}$ is the number of rows of of $A_{\delta i}$. This error is asymptotically stable [1]. The adaptive observer design as presented above can estimate both the states and the uncertainty, given that the uncertainty is observable from the available measurements. In the case when all the states are measured, the adaptive observer concept can be used to estimate uncertainty alone. In the following section, the adaptive observer is used in estimating uncertainty in the state matrices of the 2-DOF robot arm fuzzy model, given all its states are measured. 


\section{5-2 Adaptive observer design for the simplified 2-DOF robot arm}

The adaptive observer is designed for the 2-DOF robot arm for which TS fuzzy model is given in Section 3-5. Uncertainty is assumed to be in the state matrices of the fuzzy model. An uncertainty in any of the parameters $P_{1}, P_{2}, P_{3}, b_{1}$ or $b_{2}$ can essentially result in uncertainty in the positions $(1,1),(1,2),(2,1)$ or $(2,2)$ of the state matrices (3-35)-(3-38) based on the presence of the above mentioned parameters in those positions. For the purpose of adaptive observer design, a constant uncertainty is assumed to exist in the position $(1,2)$ of the state matrices which can be attributed to an uncertainty in the parameter $b_{2}$. As a simplification, the constant uncertainty value is assumed to be the same in all the rules. The simulations can as well be performed with constant uncertainty in any position of the state matrices with appropriate $M_{i}$ matrices. To test the adaptive observer, simulations are performed. The results are presented in this section.

For the purpose of simulations, the constant uncertainty is assumed to be in position $(1,2)$ of the state matrices (i.e., in the position of $A$ matrix which multiplies the state $\dot{\theta}_{2}$ in the equation for the dynamics of $\theta_{1}$ ). For example, the uncertainty matrix for the four state robot arm model can be

$$
\Delta A_{i}=\left[\begin{array}{cccc}
0 & c_{12} & 0 & 0 \\
0 & 0 & 0 & 0 \\
0 & 0 & 0 & 0 \\
0 & 0 & 0 & 0
\end{array}\right]
$$

where $c_{12}$ represents the constant uncertainty in the position $(1,2)$ of the state matrix. To estimate the uncertainty in the first row of the state matrix, the disturbance distribution matrix $M_{i}=\left[\begin{array}{llll}1 & 0 & 0 & 0\end{array}\right]^{T}$ and $A_{\delta i}$ is chosen to be a row matrix such that the product $M_{i} \widehat{A}_{\delta i}$ gives the uncertainty estimate for the state matrices i.e., $\Delta A_{i}$. In the following, the simulation results are presented for constant uncertainty of $c_{12}=0.6$ and $\mu_{\max }=2$.

The uncertainty in the state matrices is $c_{12}=0.6$ in all the rules. The simulation is run for $50 \mathrm{~s}$ and the uncertainty estimates are stored. Continuous execution of the adaptive observer for $50 \mathrm{~s}$ is referred to as one run of the adaptive observer. The input used to excite the system is a multisine signal limited to $3 \mathrm{~Hz}$. The angles are limited to $-1.6 \leq \theta_{1} \leq 1.6$ (radians) and $-1.7 \leq \theta_{2} \leq 1.64$ (radians). The initial states of the TS fuzzy model is $\boldsymbol{x}(0)_{\text {deg }}=\left[\begin{array}{llll}28.6479 & -40.1070 & -9.7403 & 14.8969\end{array}\right]^{T}$. The initial conditions for the state of the adaptive observer is $\boldsymbol{x}(0)_{d e g}=\left[\begin{array}{llll}0 & 0 & 0 & 0\end{array}\right]^{T}$. The uncertainty in all the rules $A_{\delta i}=$ $\left[\begin{array}{llll}0 & 0.6 & 0 & 0\end{array}\right]$ and the initial estimate of the uncertainty $\widehat{A}_{\delta i}=\left[\begin{array}{llll}0 & 0 & 0 & 0\end{array}\right]$. 


\section{Case 1}

In this case, the adaptive observer is designed ${ }^{6}$ for $\mu_{\max }=2$. The values of the matrices $P$ and the observer gains $L_{i}, i=1,2, \ldots, r$ are obtained by solving the LMIs $^{7}$

$$
\begin{array}{r}
\mathcal{H}\left(P\left(A_{i}-L_{i} C\right)\right)<-Q \\
{\left[\begin{array}{cc}
Q-\mu_{\text {max }}^{2} I & P \\
P & -I
\end{array}\right]}
\end{array}
$$

The value of the $P$ matrix is obtained as

$$
P=\left[\begin{array}{cccc}
0.873 & 0 & 0 & 0 \\
0 & 0.8730 & 0 & 0 \\
0 & 0 & 0.8730 & 0 \\
0 & 0 & 0 & 0.8730
\end{array}\right]
$$

and the observer gains $L_{i}, i=1,2, \ldots, r$ are

$$
\begin{aligned}
L_{1} & =\left[\begin{array}{cccc}
-53.5256 & 39.5860 & 0.5143 & -0.0347 \\
38.6621 & -618.9929 & -0.0268 & 0.5034 \\
0.4857 & 0.0268 & 10.2487 & 0.0001 \\
0.0347 & 0.4966 & -0.0001 & 10.2487
\end{array}\right] \\
L_{2} & =\left[\begin{array}{cccc}
-53.5256 & 39.0387 & 0.4925 & 0.0793 \\
37.0730 & -618.2889 & 0.0454 & 0.4952 \\
0.5075 & -0.0454 & 10.2487 & 0.0000 \\
-0.0793 & 0.5048 & 0.0000 & 10.2487
\end{array}\right] \\
L_{3} & =\left[\begin{array}{cccc}
-53.5248 & 38.9245 & 0.5364 & 0.0464 \\
39.3225 & -618.9850 & -0.0931 & 0.4705 \\
0.4636 & 0.0931 & 10.2487 & -0.0001 \\
-0.0464 & 0.5295 & 0.0001 & 10.2487
\end{array}\right] \\
L_{4} & =\left[\begin{array}{cccc}
-53.5248 & 38.0612 & 0.5265 & 0.0021 \\
38.0495 & -618.2810 & 0.0000 & 0.4999 \\
0.4735 & 0.0000 & 10.2487 & 0.0000 \\
-0.0021 & 0.5001 & 0.0000 & 10.2487
\end{array}\right]
\end{aligned}
$$

After executing the adaptive observer for $50 \mathrm{~s}$, the uncertainty estimates obtained are:

$$
\begin{aligned}
\widehat{A}_{\delta 1} & =\left[\begin{array}{llll}
0.0077 & 0.5863 & -0.0002 & -0.0097
\end{array}\right] \\
\widehat{A}_{\delta 2} & =\left[\begin{array}{llll}
0.0028 & 0.0670 & -0.0008 & -0.0042
\end{array}\right] \\
\widehat{A}_{\delta 3} & =\left[\begin{array}{llll}
0.0070 & 0.1832 & -0.0028 & -0.0138
\end{array}\right] \\
\widehat{A}_{\delta 4} & =\left[\begin{array}{llll}
0.0002 & 0.0559 & -0.0020 & -0.0084
\end{array}\right]
\end{aligned}
$$

It can be seen from the values of the uncertainty estimates, that the adaptive observer introduces non-zero estimated values for all the elements in $\widehat{A}_{\delta i}$ (not only the element in position

\footnotetext{
${ }^{6}$ I thank dr. ir. Zsófia Lendek for providing a reference Simulink model for adaptive observer.

${ }^{7}$ The system of LMIs is solved using 'feasp' function of MATLAB's LMI toolbox. 
$(1,2))$. However, the value at position $(1,2)$ in the uncertainty estimates of all the rules are considerably higher than the other elements.

Figure 5-1 presents the input signal used to excite the TS fuzzy model. Figure 5-2 gives the activation of the rules with this input when $\left\|A_{\delta i}\right\|=0.6$. The input in Figure 5-1 ensures that all rules are activated. The activation of rule $R_{1}$ is close to 1 for most of the simulation. The activation of rule $R_{2}$ is very small. From this, it can be expected that the uncertainty estimation error goes to 0 faster in case of the local model $R_{1}$ compared to the other local models. Figure 5-3 presents the value of $\operatorname{trace}\left(\bar{A}_{\delta i}^{T} \bar{A}_{\delta i}\right)$ for each of the local linear models,
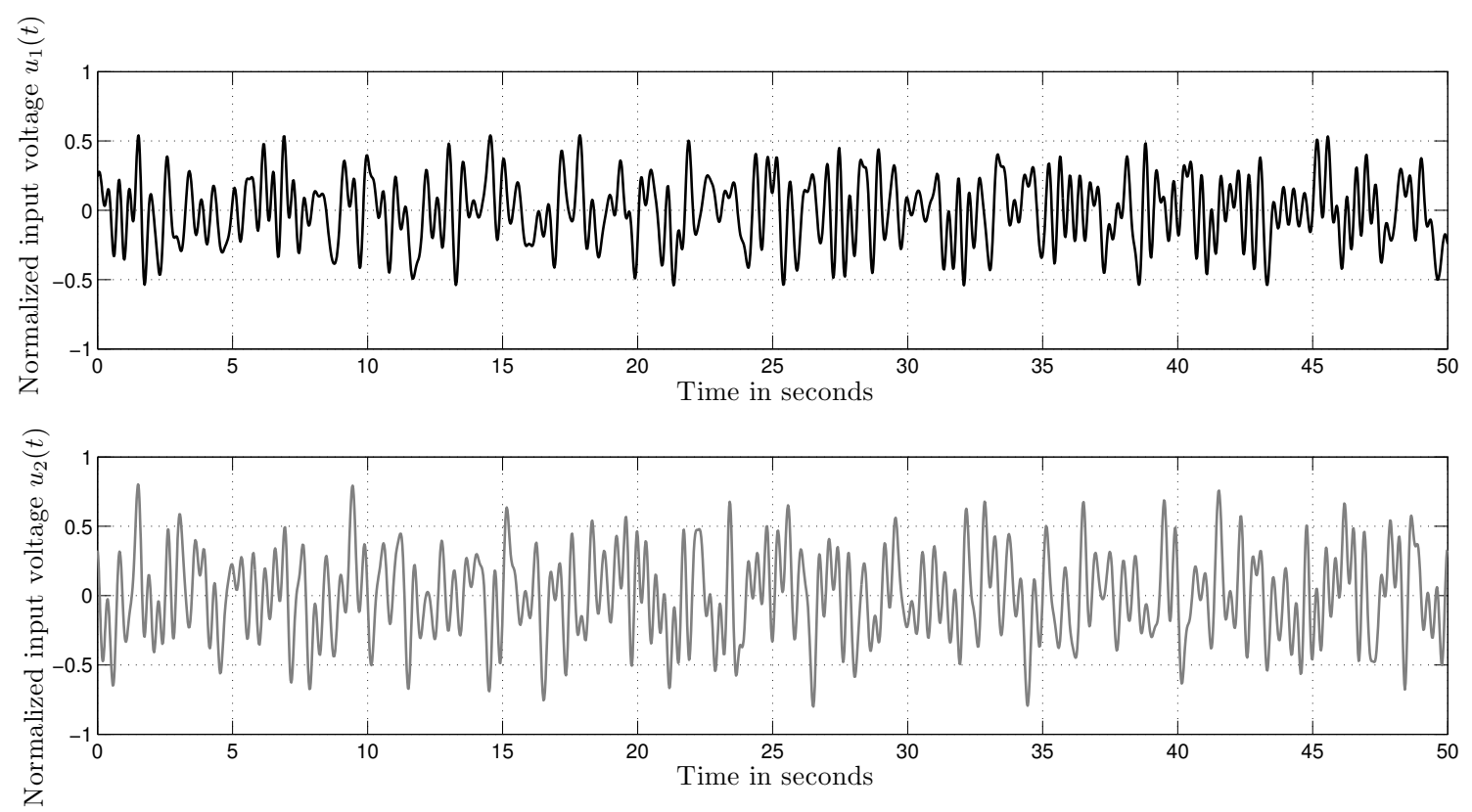

Figure 5-1: Input used to estimate uncertainty.

where $\bar{A}_{\delta i}=A_{\delta i}-\widehat{A}_{\delta i}$. The uncertainty estimation error is defined as $\sum_{i=1}^{r} \operatorname{trace}\left(\bar{A}_{\delta i}^{T} \bar{A}_{\delta i}\right)[1]$. The trace corresponding to the individual rules are presented in Figure 5-3, the sum of which gives the total uncertainty estimation error. It can be seen that the uncertainty estimation error reaches smaller values for the rule which has a high value of activation (for e.g. $R_{1}$ ). The difference between the real uncertainty and the estimated uncertainty is referred to as residual uncertainty and its norm for each local model is shown in Figure 5-5.

Figure 5-4 presents the state estimation error when $\left\|A_{\delta i}\right\|=0.6$ and $\mu_{\max }=2$. Though this is not much of consequence in the case where all the states are measured, analyzing this plot indicates speed of state and uncertainty estimation with respect to changing input. 

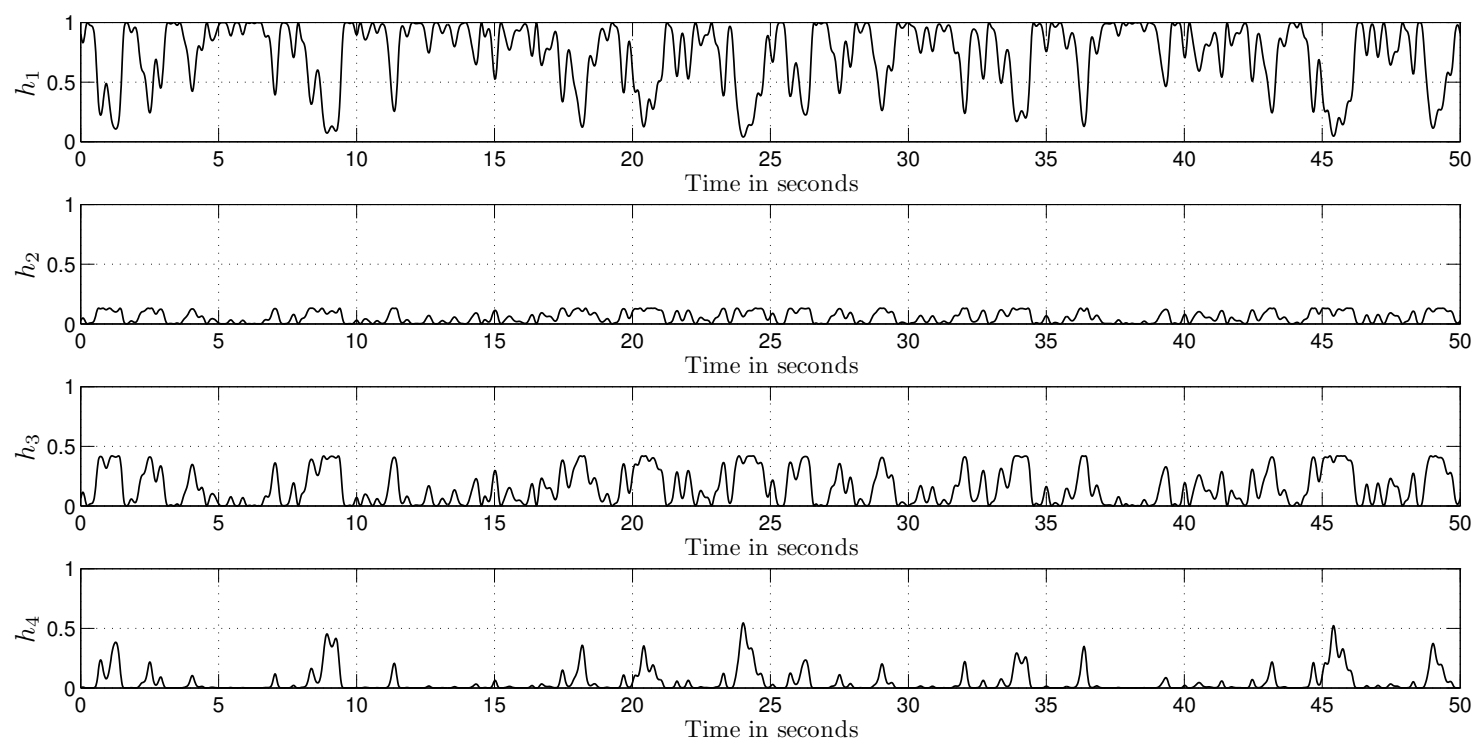

Figure 5-2: Activation of the rules with the input used.
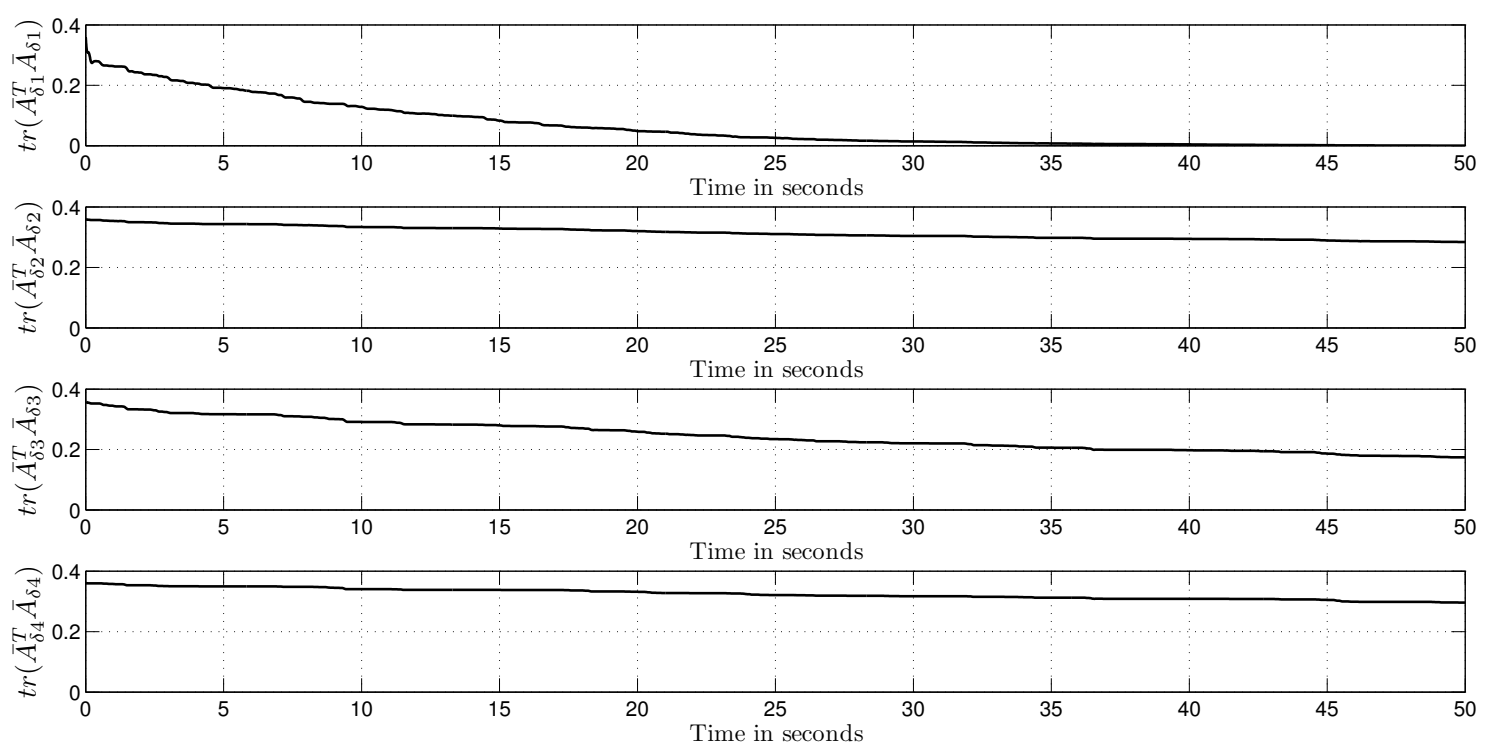

Figure 5-3: Uncertainty estimation error with $\left\|A_{\delta i}\right\|=0.6$ and $\mu_{\max }=2$.

\section{Case 2}

In this case, the adaptive observer is designed for $\mu_{\max }=20$. The value of the matrix $P$ is obtained as

$$
P=\left[\begin{array}{cccc}
14.6736 & 0 & 0 & 0 \\
0 & 14.6736 & 0 & 0 \\
0 & 0 & 14.6736 & 0 \\
0 & 0 & 0 & 14.6736
\end{array}\right]
$$



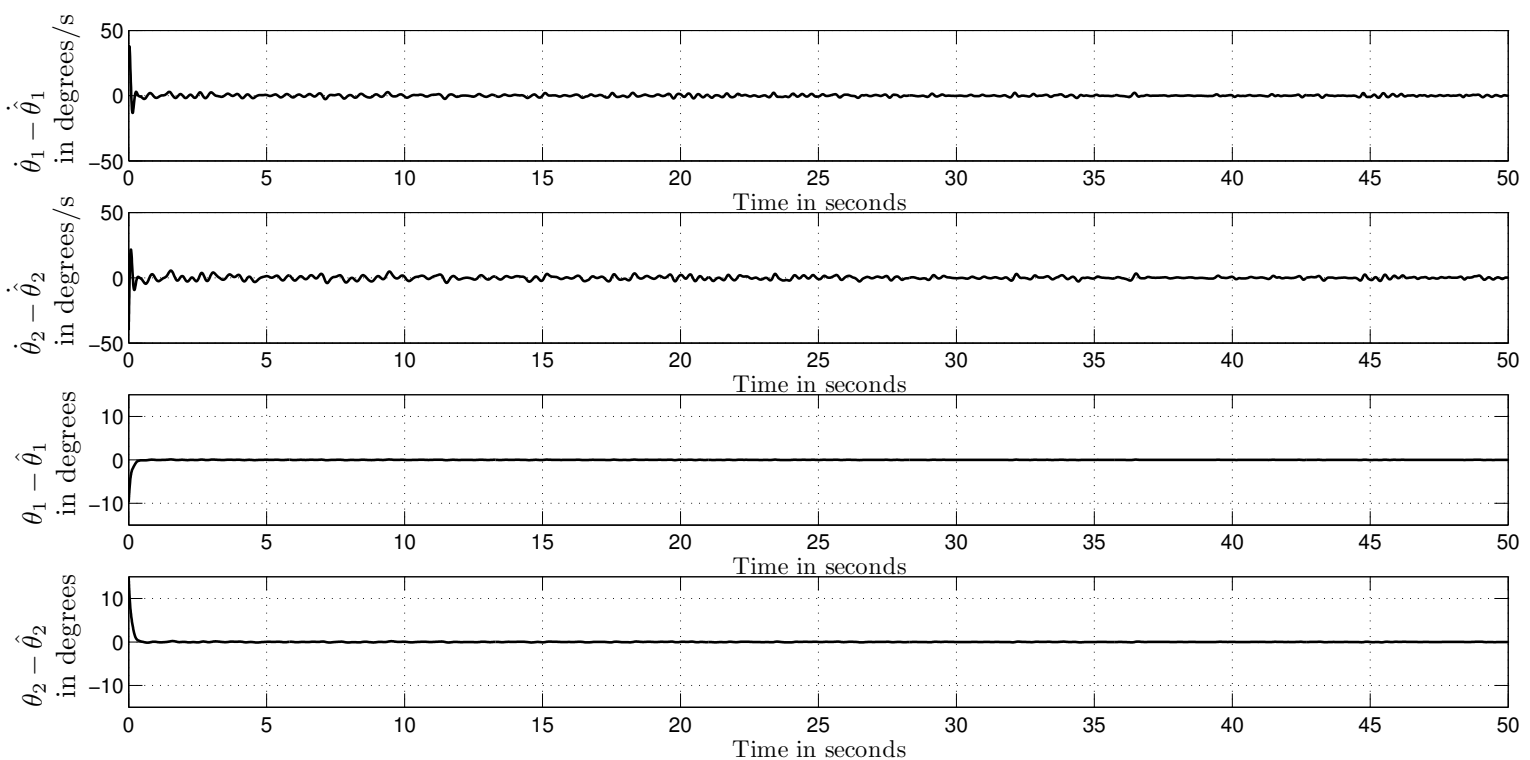

Figure 5-4: State estimation error with $\left\|A_{\delta i}\right\|=0.6$ and $\mu_{\max }=2$.
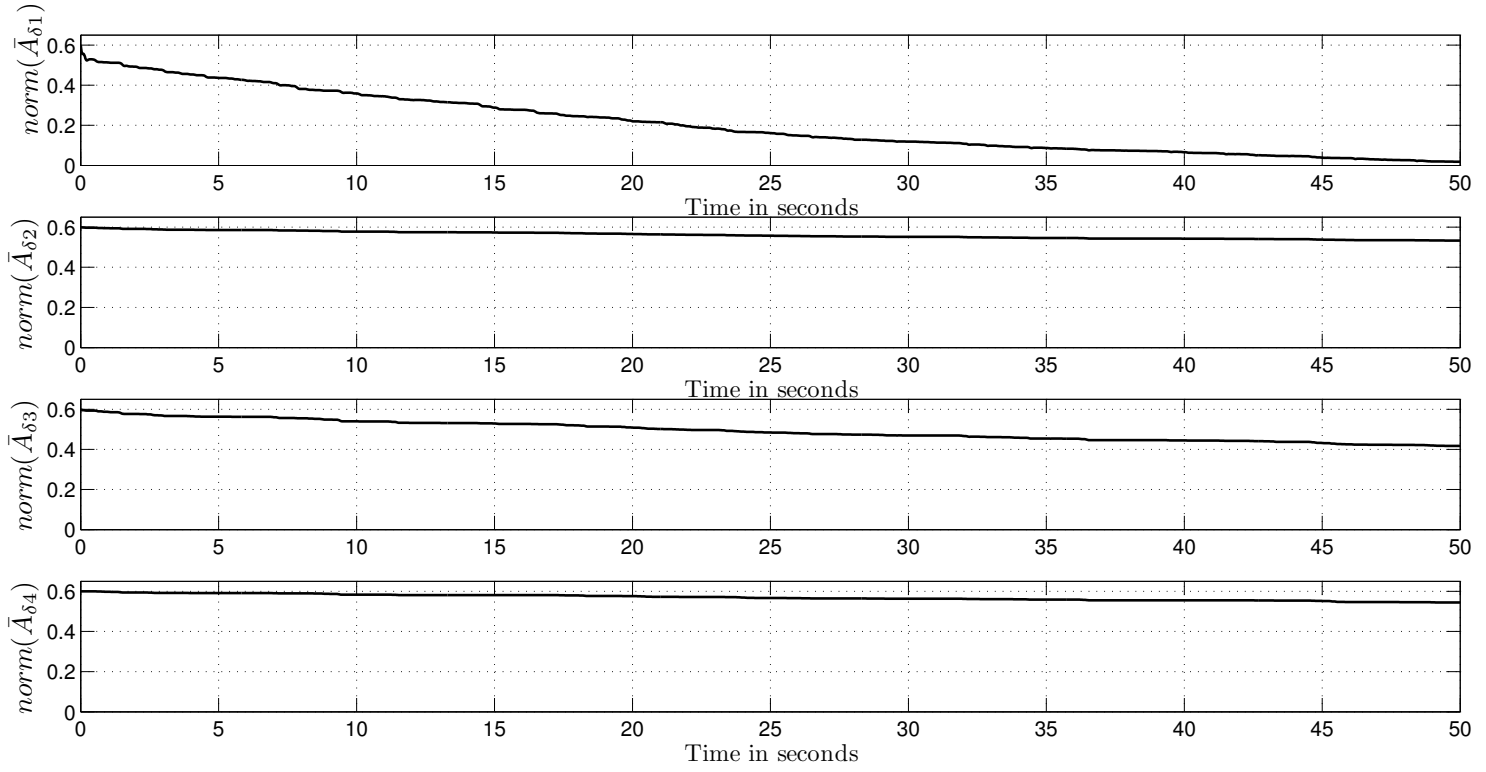

Figure 5-5: Norm of the residual uncertainty $\left\|A_{\delta i}\right\|=0.6$ and $\mu_{\max }=2$.

and the observer gains $L_{i}, i=1,2, \ldots, r$ are

$$
L_{1}=\left[\begin{array}{cccc}
9.0613 & 39.1250 & 0.4999 & 0.0000 \\
39.1230 & -556.4060 & 0.0000 & 0.5000 \\
0.5001 & 0.0000 & 72.8356 & 0.0000 \\
0.0000 & 0.5000 & 0.0000 & 72.8356
\end{array}\right]
$$




$$
\begin{aligned}
L_{2} & =\left[\begin{array}{cccc}
9.0613 & 38.0570 & 0.4999 & 0.0000 \\
38.0547 & -555.7020 & 0.0000 & 0.5000 \\
0.5001 & 0.0000 & 72.8356 & 0.0000 \\
0.0000 & 0.5000 & 0.0000 & 72.8356
\end{array}\right] \\
L_{3} & =\left[\begin{array}{cccc}
9.0621 & 39.1230 & 0.5000 & 0.0000 \\
39.1241 & -556.3981 & -0.0001 & 0.5000 \\
0.5000 & 0.0001 & 72.8356 & 0.0000 \\
0.0000 & 0.5000 & 0.0000 & 72.8356
\end{array}\right] \\
L_{4} & =\left[\begin{array}{cccc}
9.0621 & 38.0553 & 0.5000 & 0.0000 \\
38.0554 & -555.6941 & 0.0000 & 0.5000 \\
0.5000 & 0.0000 & 72.8356 & 0.0000 \\
0.0000 & 0.5000 & 0.0000 & 72.8356
\end{array}\right]
\end{aligned}
$$

After executing the adaptive observer for $50 \mathrm{~s}$, the uncertainty estimates obtained are:

$$
\begin{aligned}
\widehat{A}_{\delta 1} & =\left[\begin{array}{llll}
-0.0077 & 0.6351 & 0.0010 & 0.0149
\end{array}\right] \\
\widehat{A}_{\delta 2} & =\left[\begin{array}{llll}
0.0168 & 0.2033 & -0.0010 & 0.0024
\end{array}\right] \\
\widehat{A}_{\delta 3} & =\left[\begin{array}{llll}
0.0478 & 0.5868 & -0.0028 & 0.0084
\end{array}\right] \\
\widehat{A}_{\delta 4} & =\left[\begin{array}{llll}
0.0168 & 0.2285 & -0.0012 & 0.0059
\end{array}\right]
\end{aligned}
$$

The input used in this case is same as in Figure 5-1. Rule activations remain the same (Figure 5-2). Hence, even in this case it is expected for the uncertainty estimation error to go to 0 faster in case of the local model of the rule $R_{1}$ than in case of the other local models. Figure 5-6 shows that the uncertainty estimation error reduces at a faster rate than with $\mu_{\max }=2$. Similarly, Figure 5-7 shows that the state estimation error reduces faster than with $\mu_{\max }=2$. Figure 5-8 presents the norm of the residual uncertainty.

\section{Case 3}

In this case, the adaptive observer is designed for $\mu_{\max }=200$.

The value of the $P$ matrix is obtained as

$$
P=\left[\begin{array}{cccc}
557.8511 & 0.0179 & 0.0004 & 0.0000 \\
0.0179 & 557.6912 & 0.0000 & 0.0000 \\
0.0004 & 0.0000 & 557.9063 & 0.0000 \\
0.0000 & 0.0000 & 0.0000 & 557.9063
\end{array}\right]
$$

and the observer gains $L_{i}, i=1,2, \ldots, r$ are

$$
L_{1}=\left[\begin{array}{cccc}
2681.5 & 39.0674 & 0.4986 & -9.2237 \cdot 10^{-5} \\
39.0605 & 2116.5 & -1.5034 \cdot 10^{-4} & 0.5002 \\
0.4986 & -1.2659 \cdot 10^{-4} & 2745.1 & -2.9558 \cdot 10^{-8} \\
-7.5630 \cdot 10^{-5} & 0.5000 & -1.8731 \cdot 10^{-8} & 2745.1
\end{array}\right]
$$



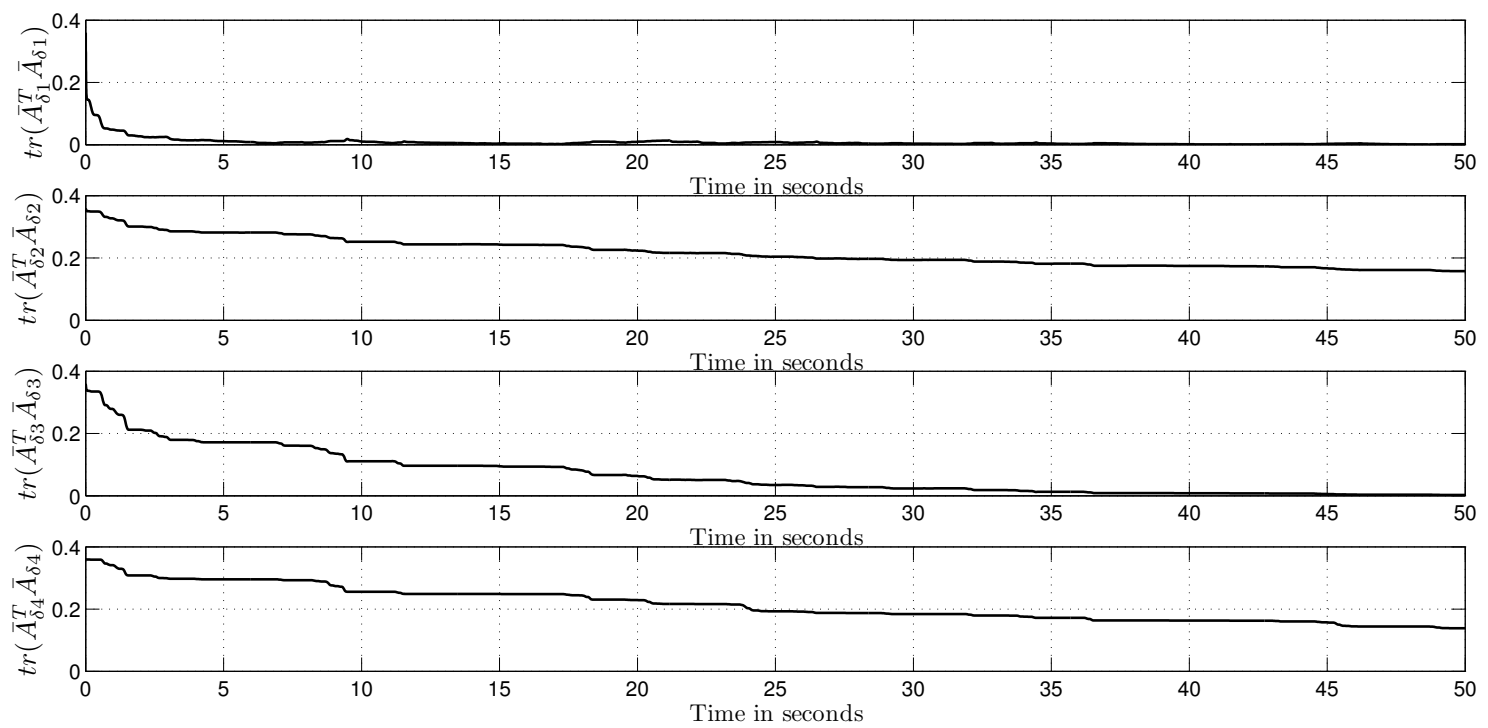

Figure 5-6: Uncertainty estimation error with uncertainty norm $=0.6$ and $\mu_{\max }=20$.
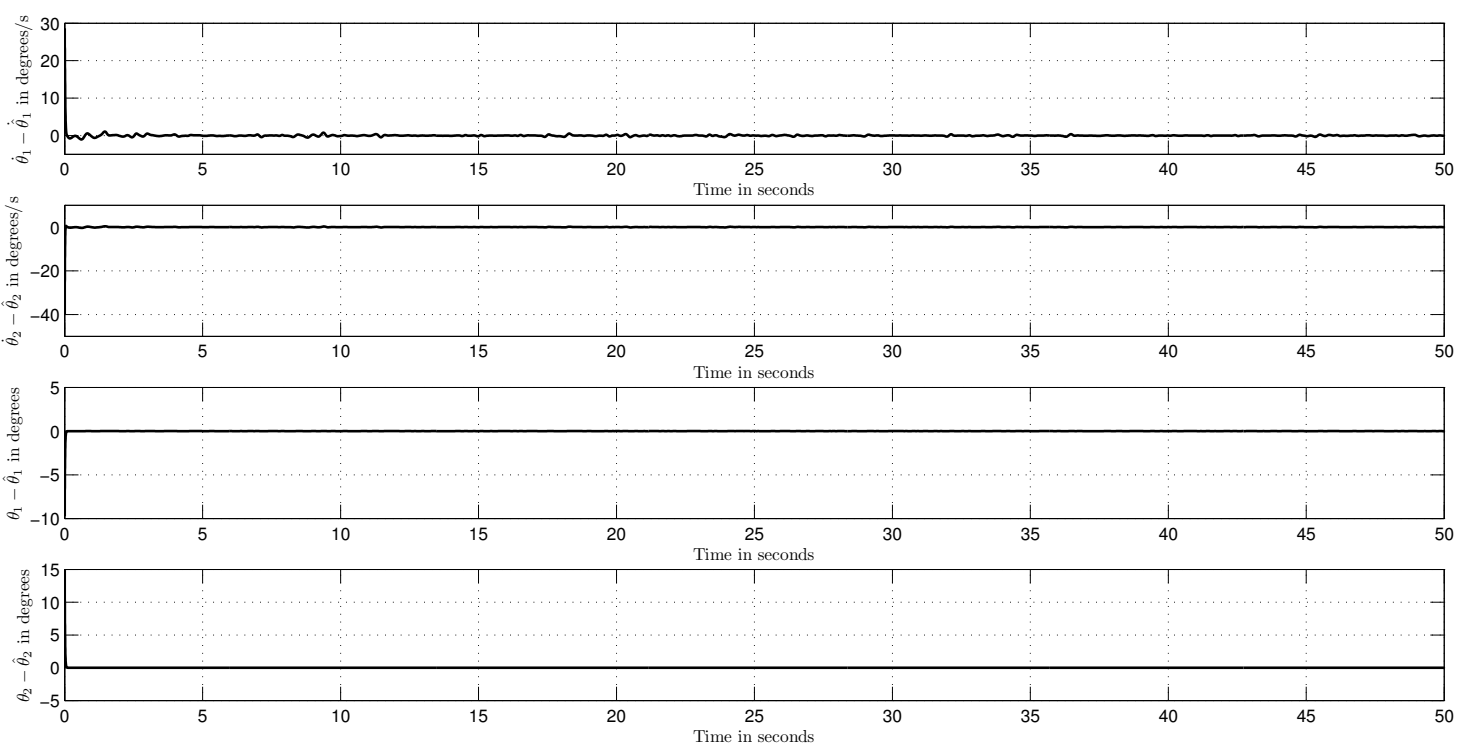

Figure 5-7: State estimation error with $\left\|A_{\delta i}\right\|=0.6$ and $\mu_{\max }=20$.

8

$$
L_{2}=\left[\begin{array}{cccc}
2681.5 & 37.9994 & 0.4986 & -9.227 \cdot 10^{-5} \\
37.9922 & 2117.2 & -1.5045 \cdot 10^{-4} & 0.5002 \\
0.4986 & -1.2594 \cdot 10^{-4} & 2745.1 & -2.9557 \cdot 10^{-8} \\
-7.5630 \cdot 10^{-5} & 0.5000 & -1.8733 \cdot 10^{-8} & 2745.1
\end{array}\right]
$$



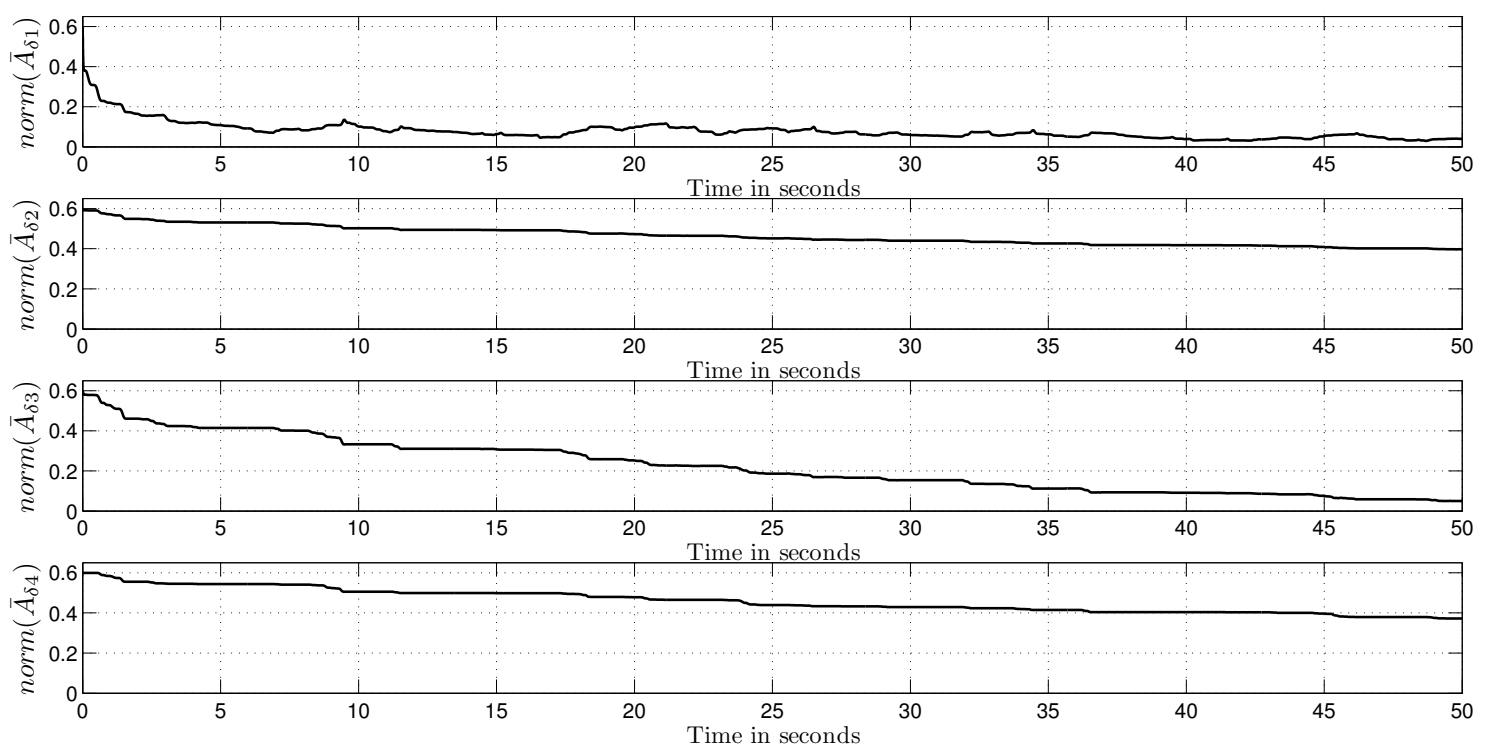

Figure 5-8: Norm of the residual uncertainty $\left\|A_{\delta i}\right\|=0.6$ and $\mu_{\max }=20$.

$$
\begin{aligned}
L_{3} & =\left[\begin{array}{cccc}
2681.5 & 39.0669 & 0.4986 & -9.223 \cdot 10^{-5} \\
39.0600 & 2116.5 & -1.5035 \cdot 10^{-4} & 0.5002 \\
0.4986 & -1.2658 \cdot 10^{-4} & 2745.1 & -2.9555 \cdot 10^{-8} \\
-7.5637 \cdot 10^{-5} & 0.5000 & -1.8734 \cdot 10^{-8} & 2745.1
\end{array}\right] \\
L_{4} & =\left[\begin{array}{cccc}
2681.5 & 37.9989 & 0.4986 & -9.2278 \cdot 10^{-5} \\
37.9917 & 2117.2 & -1.5045 \cdot 10^{-4} & 0.5002 \\
0.4986 & -1.2594 \cdot 10^{-4} & 2745.1 & -2.9557 \cdot 10^{-8} \\
-7.5622 \cdot 10^{-5} & 0.5000 & -1.8733 \cdot 10^{-8} & 2745.1
\end{array}\right]
\end{aligned}
$$

After executing the adaptive observer for $50 \mathrm{~s}$, the uncertainty estimates obtained are:

$$
\begin{aligned}
\widehat{A}_{\delta 1} & =\left[\begin{array}{llll}
-0.0102 & 0.6279 & 0.0034 & 0.0204
\end{array}\right] \\
\widehat{A}_{\delta 2} & =\left[\begin{array}{llll}
0.0188 & 0.2157 & -0.0009 & 0.0006
\end{array}\right] \\
\widehat{A}_{\delta 3} & =\left[\begin{array}{llll}
0.0515 & 0.6258 & -0.0031 & -0.0002
\end{array}\right] \\
\widehat{A}_{\delta 4} & =\left[\begin{array}{llll}
0.0139 & 0.2480 & -0.0025 & -0.0035
\end{array}\right]
\end{aligned}
$$

The input used in this case is same as in Figure 5-1. Rule activation is given in Figure 5-2. Figure 5-9 shows that the uncertainty estimation error reduces faster than with $\mu_{\max }=2$ and $\mu_{\max }=20$. Similarly, Figure 5-10 shows that the state estimation error reduces faster than with $\mu_{\max }=2$ and $\mu_{\max }=20$. Figure 5-11 presents the norm of the residual uncertainty during the estimation.

It can be concluded from the results of the above three cases that, if the constant uncertainty is the same and the uncertainty norm $\mu_{\max }$ for which the adaptive observer is designed is increased, the state estimation error and the uncertainty estimation error reduces at a faster 

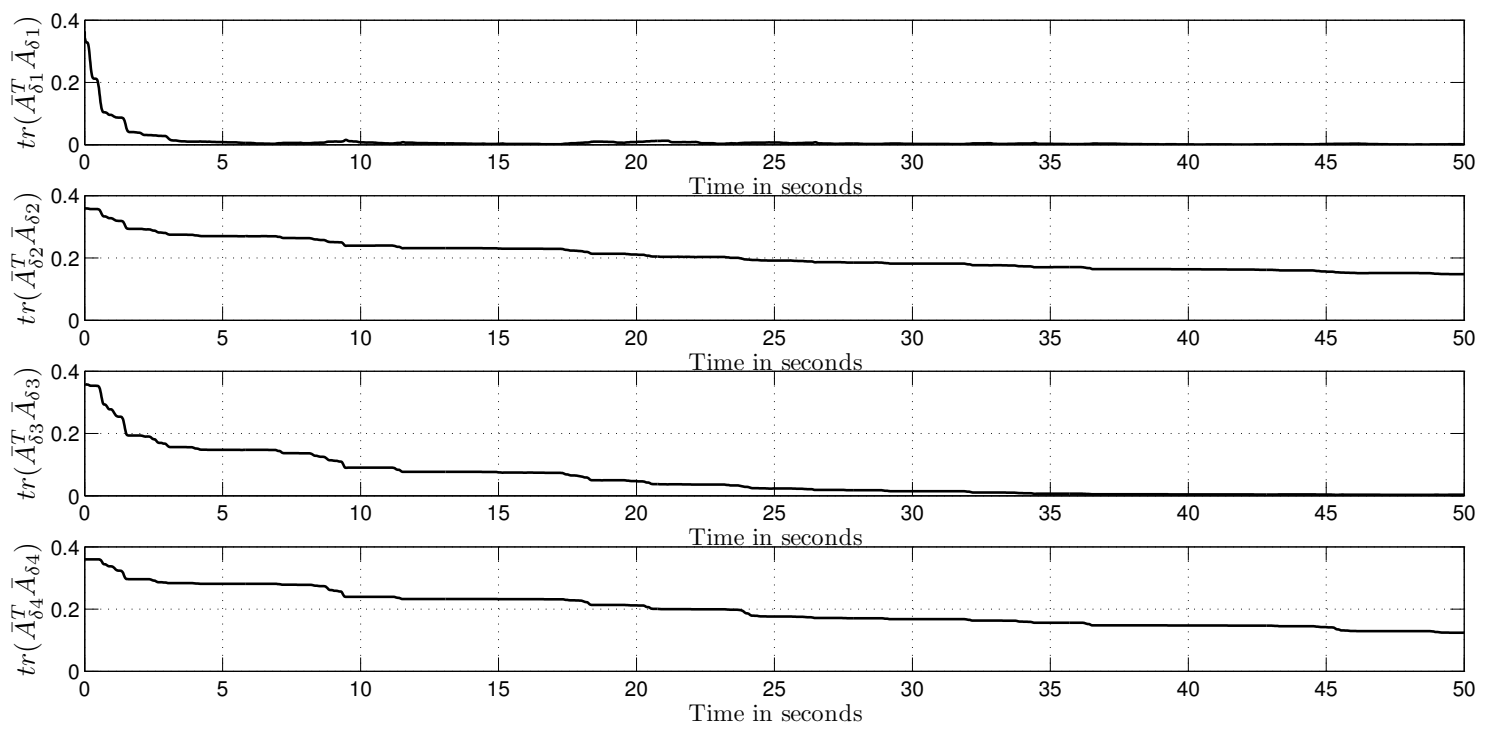

Figure 5-9: Uncertainty estimation error with $\left\|A_{\delta i}\right\|=0.6$ and $\mu_{\max }=200$.
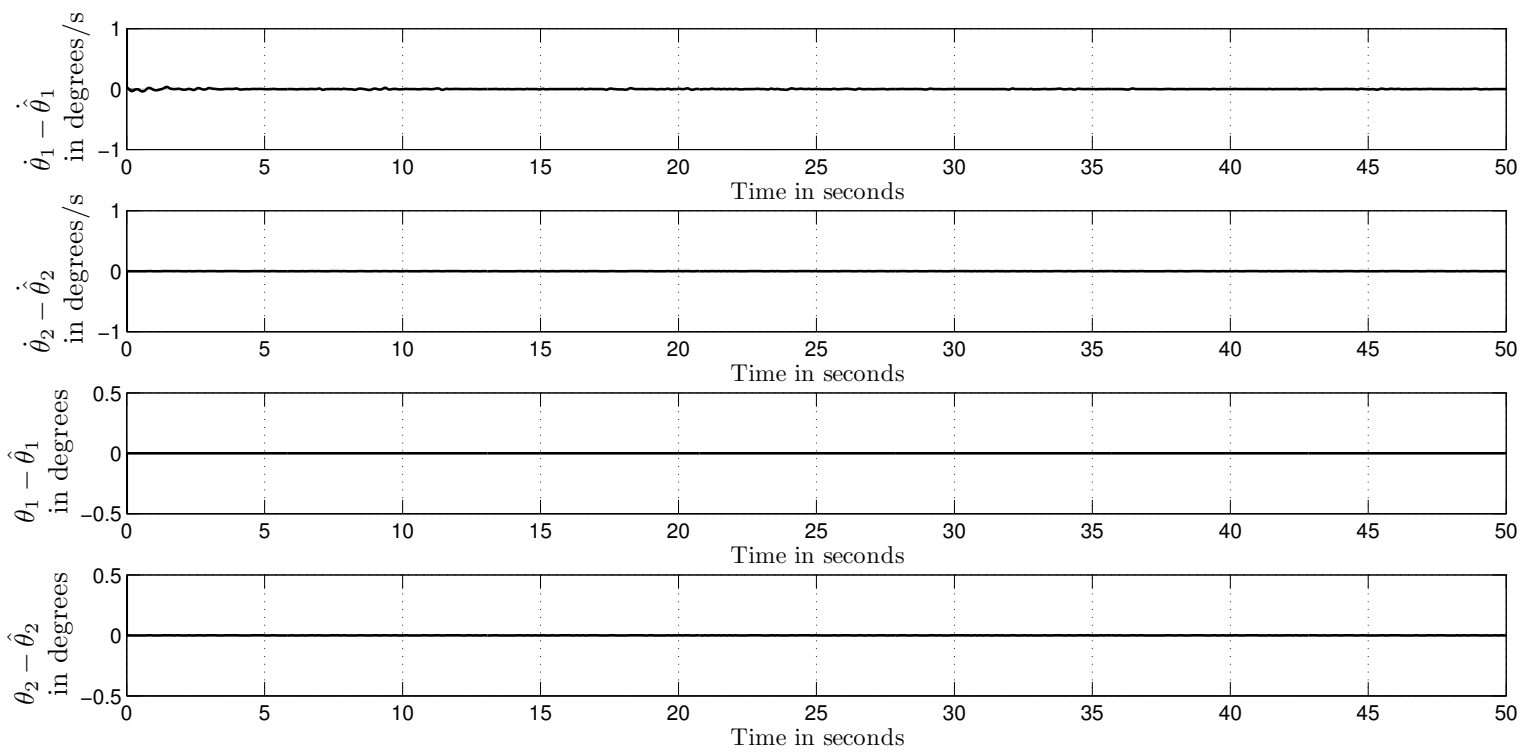

Figure 5-10: State estimation error with $\left\|A_{\delta i}\right\|=0.6$ and $\mu_{\max }=200$.

rate. Hence, $\mu_{\max }$ behaves similar to a decay rate and can be considered as the rate at which the uncertainty estimate converges to true uncertainty. It is referred to as uncertainty convergence rate from here on. The results presented in this section are with the adaptive observer being simulated for $50 \mathrm{~s}$ (referred to as one run of the adaptive observer). The uncertainty estimates obtained after the first run of the adaptive observer can be used to 

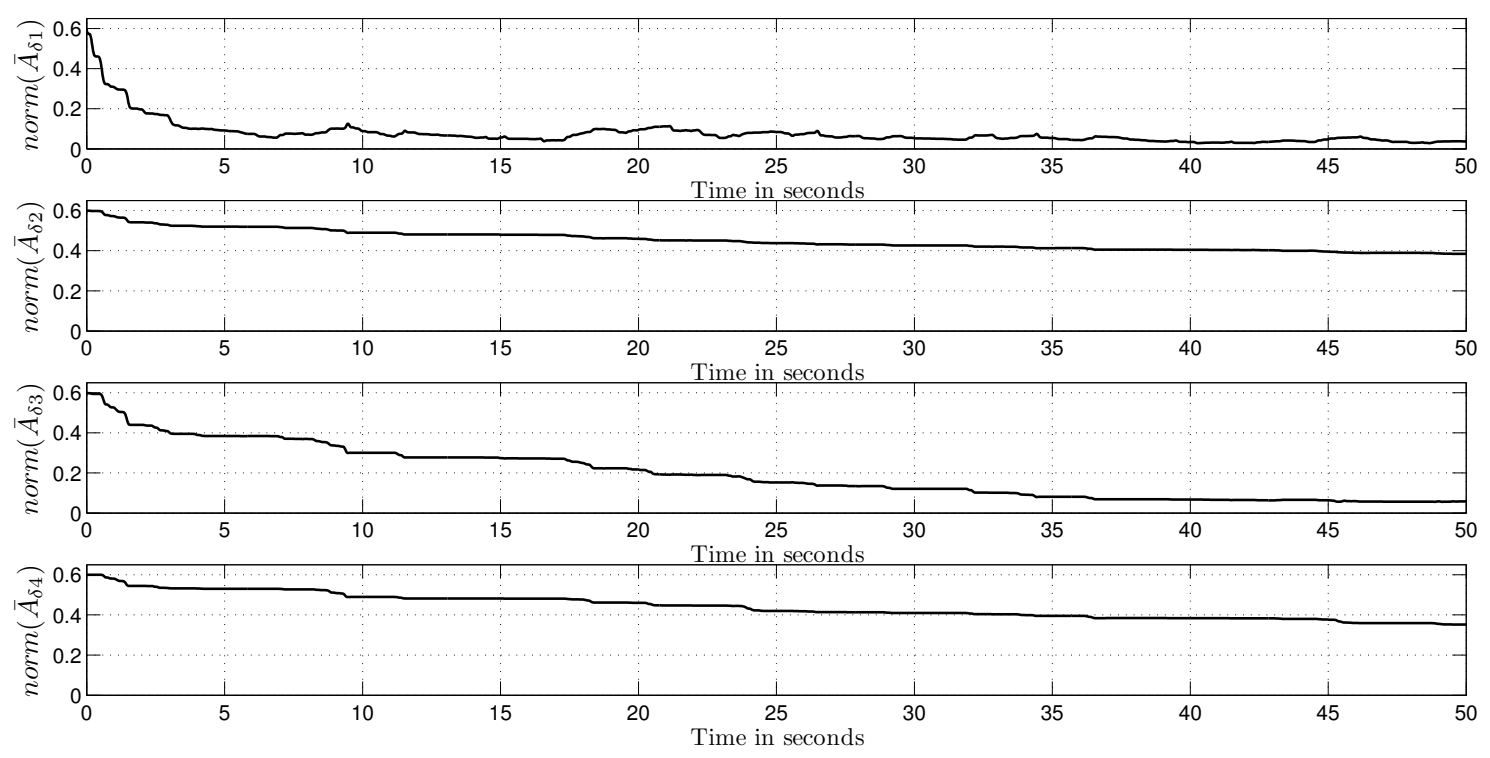

Figure 5-11: Norm of the residual uncertainty $\left\|A_{\delta i}\right\|=0.6$ and $\mu_{\max }=200$.

update the model. The adaptive observer can be used to further estimate the uncertainty in the new model. Further study is required to analyze the utility of this iterative method in better estimating the uncertainties.

The choice of input used in the experiments is based on the knowledge of the bandwidth of the operation of the model, to activate all the rules and to avoid saturation of the state variables. The time of $50 \mathrm{~s}$ for a run of the adaptive observer is experimentally determined to obtain a significant reduction in the uncertainty estimation error. The choice of the input and simulation time of the adaptive observer also influence the values of the estimates obtained. An input that ensures that all rules are sufficiently excited results in better estimation. Estimation is better in case of the rules which are active with a high degree of relevance. This is seen from the simulation results, where the uncertainty estimation error is smaller in some of the rules than the remaining ones. For instance, degree of relevance of $R_{1}$ and $R_{3}$ are higher than that of $R_{2}$ and $R_{4}$ and the uncertainty estimation error is smaller in case of $R_{1}$ and $R_{3}$ compared to $R_{2}$ and $R_{4}$. Given a suitable input, if the adaptive observer is used for a longer duration, better estimates may be obtained. The TS fuzzy model for which the uncertainty is being estimated will largely influence the possibility to activate all rules sufficiently. For example, in case of models constructed based on sector nonlinearity, it may not be possible to activate some of the rules beyond a certain degree in the defined region of state space.

This section presented the adaptive observer design for 2-DOF robot arm model and described the effect of increasing $\mu_{\max }$. The influence of the input and the duration of experiments on the uncertainty estimation is discussed. In the following section, exploiting the structure of the model in the uncertainty estimation is discussed for the specific example of 2-DOF robot arm. 


\section{5-3 Uncertainty structure information}

In the 2-DOF robot arm model, the membership functions of the TS model are dependent on the state $\theta_{2}$ only. Hence providing the input $u_{2}$ which causes variation in $\theta_{2}$ alone is sufficient to activate the rules of the model. Also considering that the constant uncertainty is present in the state matrices $A_{i}$ in the elements which multiply the states $\dot{\theta}_{2}$, only $u_{2}$ needs to be provided to estimate the uncertainty. This is illustrated on an example, with $c_{12}=0.6$ (i.e., the uncertainty multiplies the state $\dot{\theta}_{2}$ in the equation for $\ddot{\theta}_{1}$ ). The adaptive observer is designed for $\mu_{\max }=200$.

The input $u_{2}$ is presented in Figure 5-1 and the input $u_{1}$ is zero. The activations of rules are given in Figure 5-12.
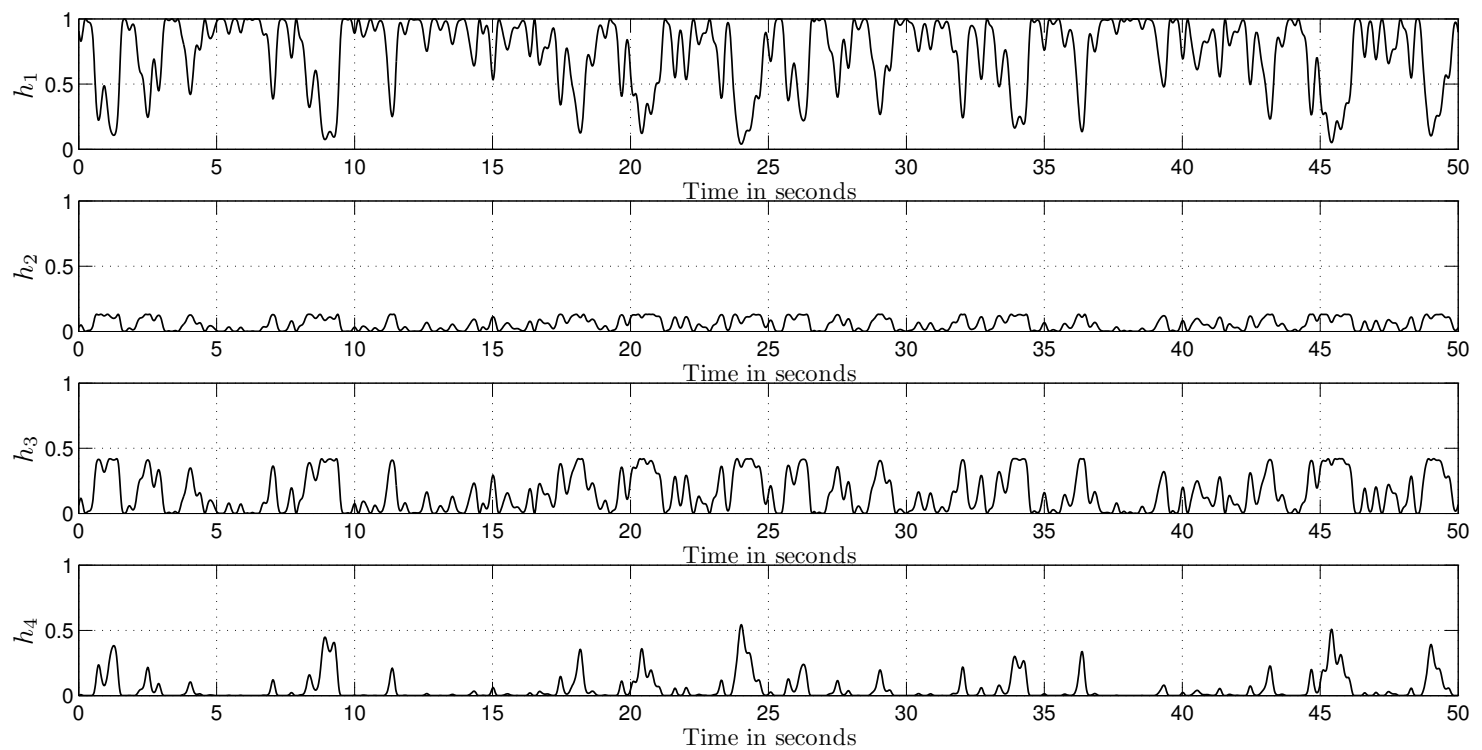

Figure 5-12: Activation of rules with the input $u_{2}$.

Figures 5-13 and 5-14 show the uncertainty estimation error and state estimation error, respectively. Figure 5-15 presents the norm of the residual uncertainty.

The uncertainty estimates after one run are obtained as

$$
\begin{aligned}
\widehat{A}_{\delta 1} & =\left[\begin{array}{llll}
0.0266 & 0.6280 & 0.0024 & 0.0191
\end{array}\right] \\
\widehat{A}_{\delta 2} & =\left[\begin{array}{llll}
0.0024 & 0.2175 & 0.0000 & 0.0007
\end{array}\right] \\
\widehat{A}_{\delta 3} & =\left[\begin{array}{llll}
0.0066 & 0.6307 & 0.0001 & 0.0011
\end{array}\right] \\
\widehat{A}_{\delta 4} & =\left[\begin{array}{llll}
0.0020 & 0.2490 & -0.0001 & -0.0012
\end{array}\right]
\end{aligned}
$$

The norms of residual uncertainties are obtained as $\left\|\bar{A}_{\delta 1}\right\|=0.0432,\left\|\bar{A}_{\delta 2}\right\|=0.3825,\left\|\bar{A}_{\delta 3}\right\|=$ 0.0314 and $\left\|\bar{A}_{\delta 4}\right\|=0.3510$. These values are close to the values obtained in the case when both inputs $u_{1}$ and $u_{2}$ are given to the model, which resulted in $\left\|\bar{A}_{\delta 1}\right\|=0.0362,\left\|\bar{A}_{\delta 2}\right\|=$ 

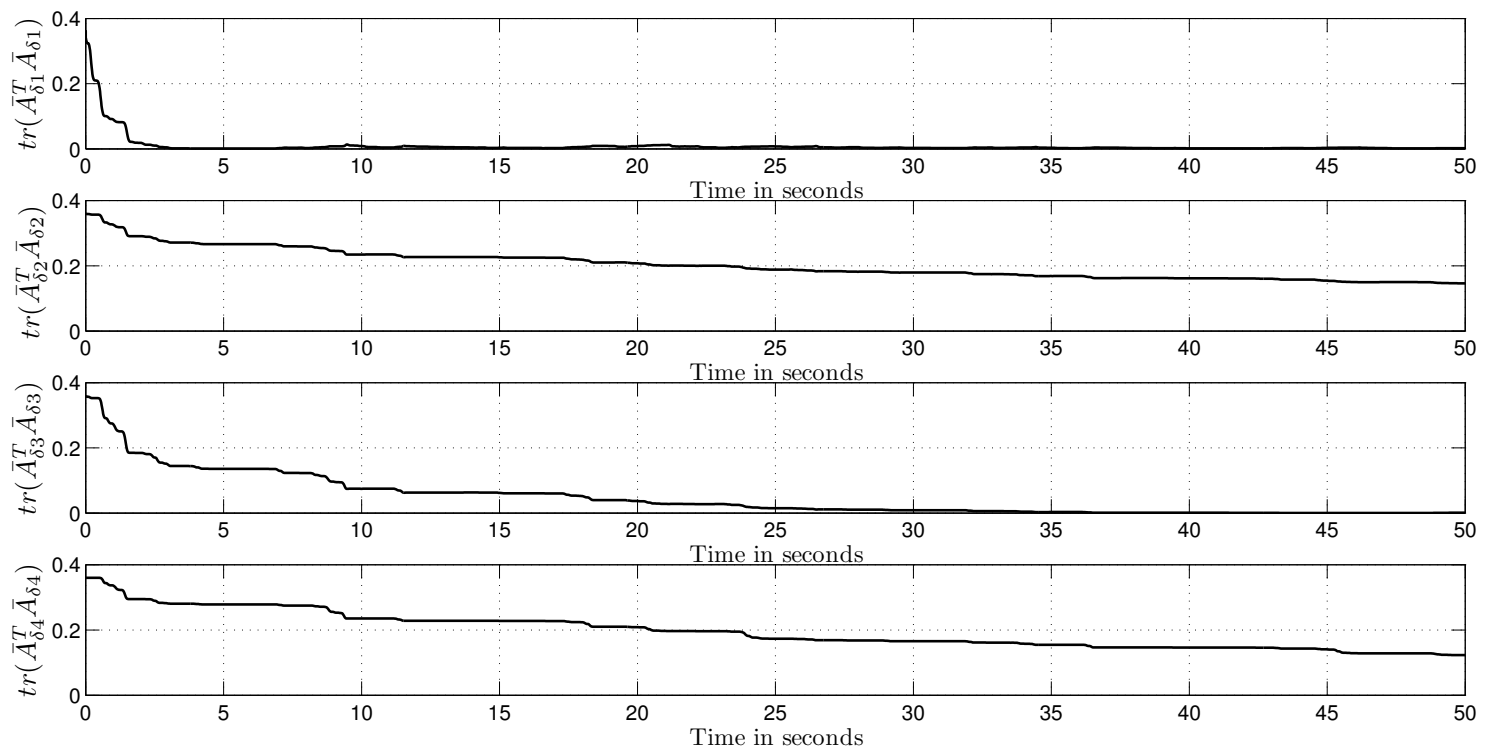

Figure 5-13: Uncertainty estimation error with $\left\|A_{\delta i}\right\|=0.6$ and $\mu_{\max }=200$.
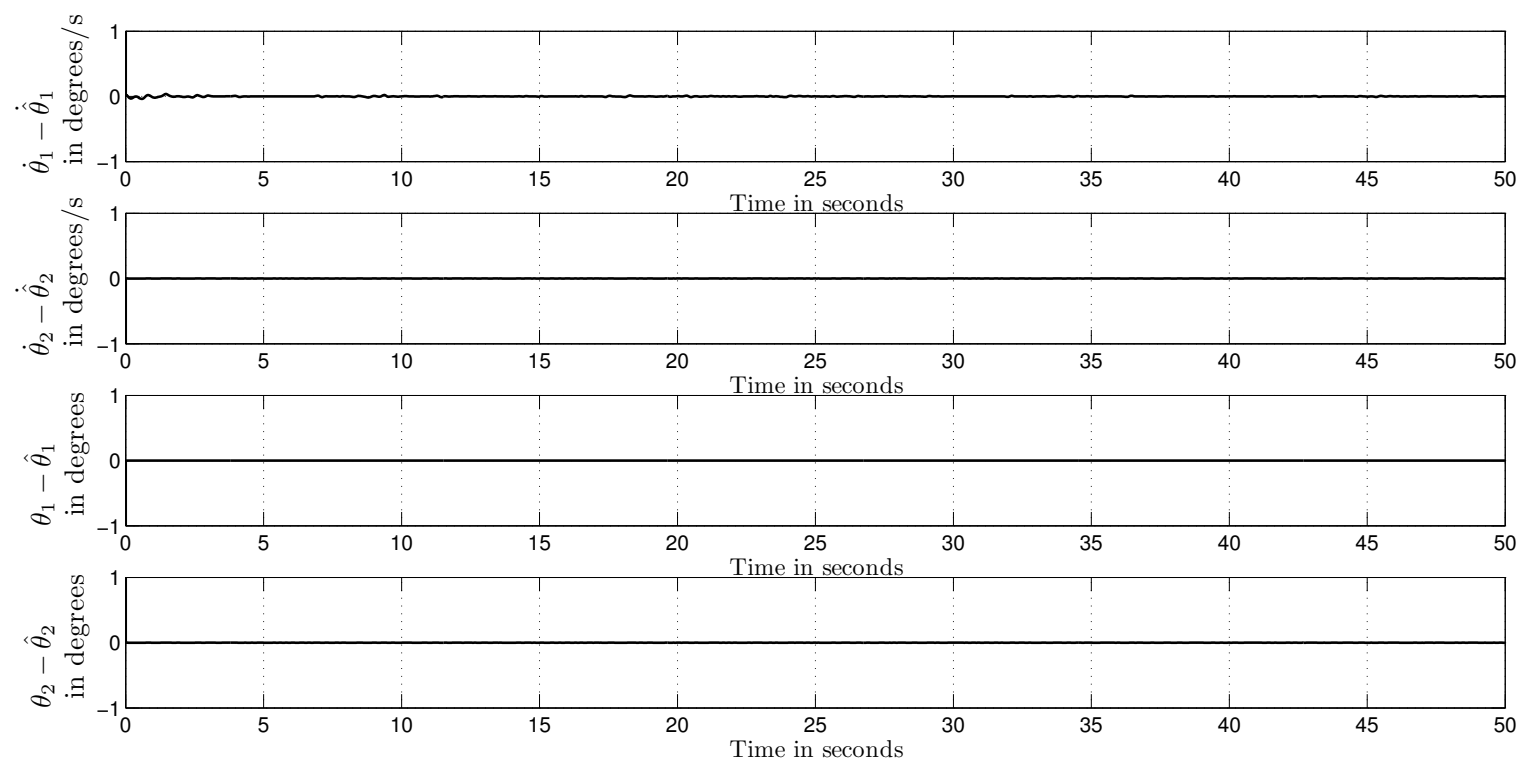

Figure 5-14: State estimation error with $\left\|A_{\delta i}\right\|=0.6$ and $\mu_{\max }=200$.

0.3847, $\left\|\bar{A}_{\delta 3}\right\|=0.0577$ and $\left\|\bar{A}_{\delta 4}\right\|=0.3523$. Hence, it can be concluded from the results that an experiment with only input $u_{2}$ can be used to estimate the uncertainties assumed to be as described above, thus avoiding the need to design the input $u_{1}$. Though considering that the uncertainty to exist only in position $(1,2)$ of the robot arm model is a simplification and far from reality, this example illustrates a possibility to use the information about the TS fuzzy model and the uncertainty structure to identify the kind of experiments to perform in order 

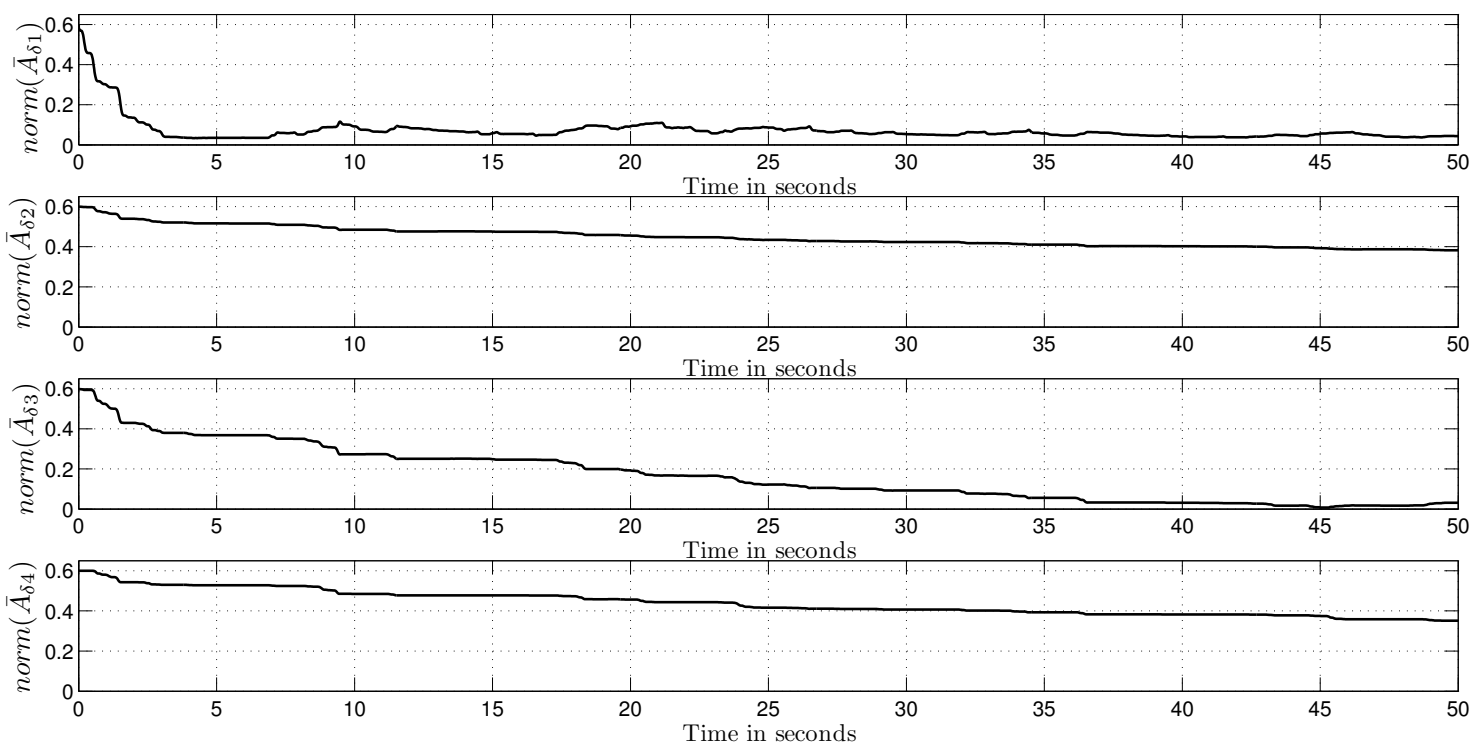

Figure 5-15: Norm of the residual uncertainty $\left\|A_{\delta i}\right\|=0.6$ and $\mu_{\max }=200$.

to estimate the uncertainties.

\section{5-4 Conclusions}

In this chapter, an adaptive observer has been designed for the 2-DOF robot arm. Given that all the states are measured, the adaptive observer has been used for the 2-DOF robot arm as an uncertainty estimator. For a constant uncertainty in the state matrices, adaptive observer designs with different values of $\mu_{\max }$ have been presented. It has been observed from the simulation results that the uncertainty block norm reduces after one run of the adaptive observer. Hence updating the initial model of the system with uncertainty estimates can result in a better model of the system. The simulation results indicate that $\mu_{\max }$ behaves as an uncertainty convergence rate. In the last section of the chapter, a possibility to exploit the information available about the uncertainty structure and the TS fuzzy model to create suitable experiments for estimating the uncertainties has been presented.

The following chapter presents the use of estimates given by the adaptive observers in the design of the robust state feedback controller. 


\section{Chapter 6}

\section{Adaptive observer and robust controller}

Chapters 4 and 5 presented the robust controller design and the adaptive observer design for Takagi-Sugeno (TS) fuzzy models to estimate the constant uncertainties in the state matrices. This chapter brings together the concepts in robust controller and adaptive observer and analyzes how the uncertainty estimates from the adaptive observer can be used in robust controller design. It has been observed in Chapter 5 that updating the initial model of the system with the uncertainty estimates provides a new model for the system with smaller uncertainty than before. Hence, there is a reason to expect improvement in the performance achievable from the controller design and this issue is analyzed in Section 6-1. Analysis is also done to see the effect of an active controller on the uncertainty estimates obtained from the adaptive observer. For this case, the system is controlled by the robust controller and an adaptive observer is active to estimate uncertainties but has no influence on the controller. This analysis is presented in Section 6-2. The chapter ends with the conclusions derived.

\section{6-1 Uncertainty estimates in controller design}

We have concluded in Chapter 5 that the adaptive observer provides uncertainty estimates which can be used to update the TS fuzzy model and thus better represent the system. However, even after updating the model with the uncertainty estimates, uncertainty can exist in the new model (depending on the residual uncertainty). Hence, a controller that can stabilize the system with uncertainty has to be designed. Since the uncertainty in the system is reduced because of the use of adaptive observer and the subsequent updating of the model, a controller can be designed with a new reduced uncertainty norm. In this section, for the 2-DOF robot arm model, it is evaluated if any improvement in controller performance can be achieved due to the availability of the new updated model from the adaptive observer. The procedure is depicted by Figure 6-1. 

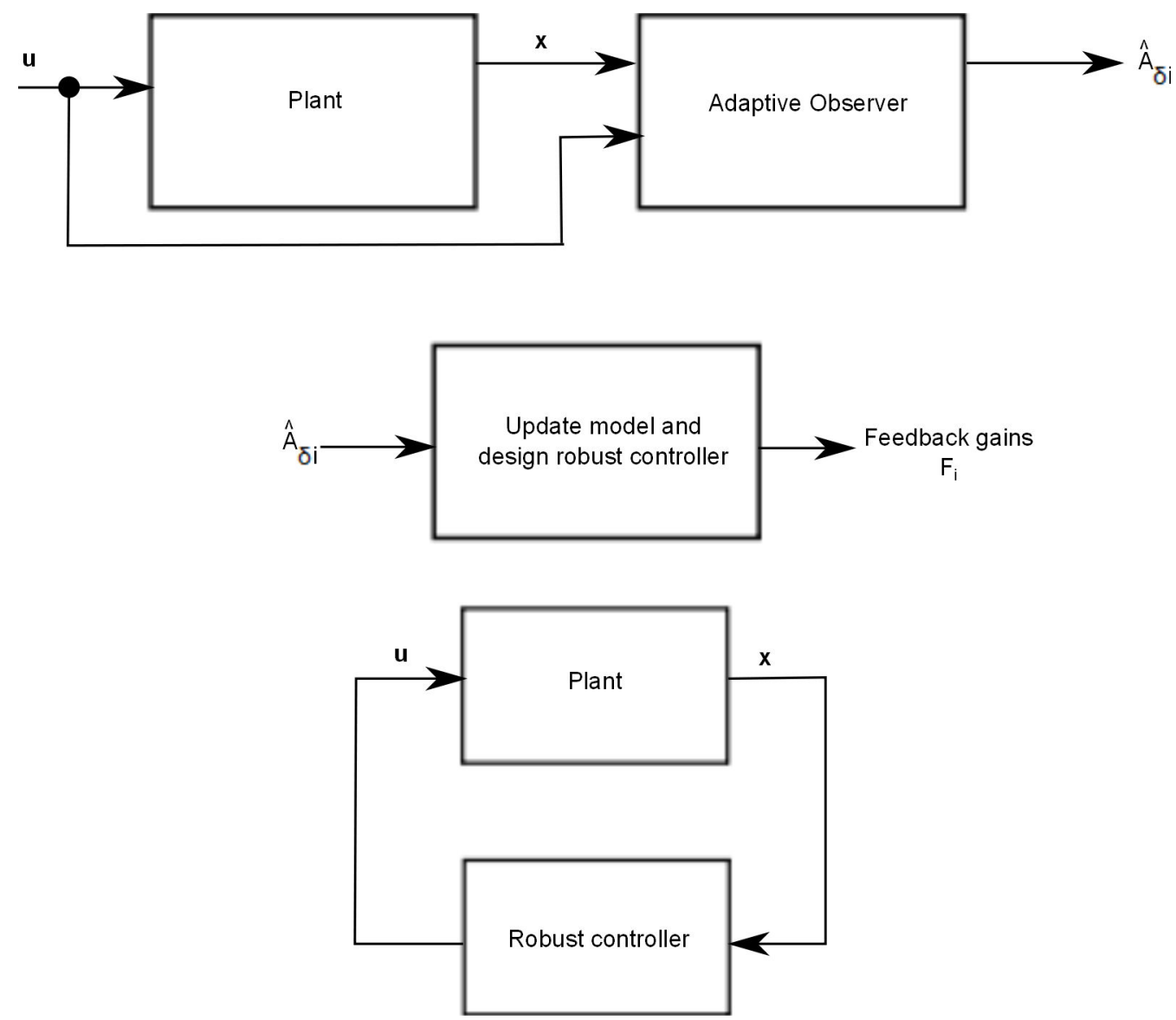

Figure 6-1: Uncertainty estimates in controller design.

In the following, the adaptive observer designed in Case 3, Section 5-2 of Chapter 5 is considered for analysis and the relevant results are presented here. Recall that in this case the uncertain TS fuzzy model is assumed to have a constant uncertainty of $c_{12}=0.6$ in the position $(1,2)$ of the state matrices in all the rules. The adaptive observer is designed for $\mu_{\max }=200$ since we obtained the best uncertainty estimates for this case among the $\mu_{\max }$ values considered. The input used is same as in Figure 5-1 in Chapter 5. The uncertainty estimates obtained after the adaptive observer was executed for $50 \mathrm{~s}$ are as follows.

$$
\begin{aligned}
\widehat{A}_{\delta 1} & =\left[\begin{array}{llll}
-0.0102 & 0.6279 & 0.0034 & 0.0204
\end{array}\right] \\
\widehat{A}_{\delta 2} & =\left[\begin{array}{llll}
0.0188 & 0.2157 & -0.0009 & 0.0006
\end{array}\right] \\
\widehat{A}_{\delta 3} & =\left[\begin{array}{llll}
0.0515 & 0.6258 & -0.0031 & -0.0002
\end{array}\right] \\
\widehat{A}_{\delta 4} & =\left[\begin{array}{llll}
0.0139 & 0.2480 & -0.0025 & -0.0035
\end{array}\right]
\end{aligned}
$$

The norms of the residual uncertainties are: $\left\|\bar{A}_{\delta 1}\right\|=0.0362,\left\|\bar{A}_{\delta 2}\right\|=0.3847,\left\|\bar{A}_{\delta 3}\right\|=0.0577$, and $\left\|\bar{A}_{\delta 4}\right\|=0.3523$. The uncertainty estimates in equation (6-1) are used in updating the TS fuzzy model. A robust controller is designed with this new updated model using Theorems 11 and 14 with the specifications of $\alpha=1, \zeta=1$, and $\phi=1$ where $\alpha$ is the decay rate, $\zeta$ is the constraint on the control input and $\phi$ is the bound on the initial state norm. The 
maximum norms on the uncertainty for which stability can be guaranteed with this controller was obtained as $\mu_{1}=\mu_{2}=\mu_{3}=\mu_{4}=0.637543$. Since the norm of the residual uncertainty is relatively smaller than these values, the value of decay rate was increased to $\alpha=2$ and the controller was redesigned. By maximizing the norms of uncertainties using Theorem 11, new values of $\mu_{1}=\mu_{2}=\mu_{3}=\mu_{4}=0.47758$ are obtained. The residual uncertainty norms are all smaller than 0.47758 and hence the controller design with $\alpha=2$ can be used to guarantee stability of the controlled system with an increased decay rate. This indicates that using the adaptive observer uncertainty estimates in updating the model of the system and redesigning the controller can guarantee stability for a higher decay rate.

If the uncertainty were to be $c_{12}=0.95$ and the design specifications to be met $\alpha=1$, $\zeta=1$, and $\phi=1$ with the initial TS fuzzy model, then the robust controller could not have guaranteed stabilization for the uncertainty norm of $\left\|A_{\delta i}\right\|=0.95$. By maximizing the uncertainty norms for which the controller can guarantee stability, the values of $\mu_{1}, \mu_{2}, \mu_{3}$, and $\mu_{4}$ are obtained to be 0.637796 . By using the adaptive observer with the same inputs as in Figure $5-1, \mu_{\max }=200$ and simulation time of $50 \mathrm{~s}$, uncertainty estimates are

$$
\begin{aligned}
\widehat{A}_{\delta 1} & =\left[\begin{array}{llll}
-0.0173 & 0.9946 & 0.0055 & 0.0284
\end{array}\right] \\
\widehat{A}_{\delta 2} & =\left[\begin{array}{llll}
0.0309 & 0.3419 & -0.0014 & 0.0011
\end{array}\right] \\
\widehat{A}_{\delta 3} & =\left[\begin{array}{llll}
0.0853 & 0.9921 & -0.0049 & 0.0004
\end{array}\right] \\
\widehat{A}_{\delta 4} & =\left[\begin{array}{llll}
00.0235 & 0.3934 & -0.0040 & -0.0051
\end{array}\right]
\end{aligned}
$$

The norms of the residual uncertainties after $50 \mathrm{~s}$ of estimation by the adaptive observer are $\left\|\bar{A}_{\delta 1}\right\|=0.0559,\left\|\bar{A}_{\delta 2}\right\|=0.6089,\left\|\bar{A}_{\delta 3}\right\|=0.0953$, and $\left\|\bar{A}_{\delta 4}\right\|=0.5571$. With the obtained values of uncertainty estimates, the TS fuzzy model is updated and a robust controller is designed with the required specifications. We get $\mu_{1}=\mu_{2}=\mu_{3}=\mu_{4}=0.6378$ which is greater than the values of the residual uncertainties and hence this robust controller can guarantee stabilization of the new TS fuzzy model.

In this section we discussed the improvement in controller performance that can be achieved by using the uncertainty estimates in the robust controller design. In the scenarios considered in this section, a controller is not active when the adaptive observer is used to estimate uncertainties. In the following section we analyze the scenario where a stabilizing controller is active and the adaptive observer works in parallel to estimate uncertainties. Again, the adaptive observer is not used to estimate states.

\section{6-2 Parallel operation of adaptive observer and robust controller}

In this section, the effect of an active robust stabilizing controller on the uncertainty estimation by adaptive observer is analyzed. The configuration used is depicted by Figure 6-2. This can be viewed from two perspectives. The first point of view is to consider the possibility where the system is unstable and hence the presence of a controller is required. Second, when the expense of having to perform open loop experiments can make the system go off-line and hence it is preferred to add an external input to perform uncertainty estimation with the system in closed loop. Both perspectives correspond to the idea of closed loop identification where an active controller is required during input-output data generating experiments. 


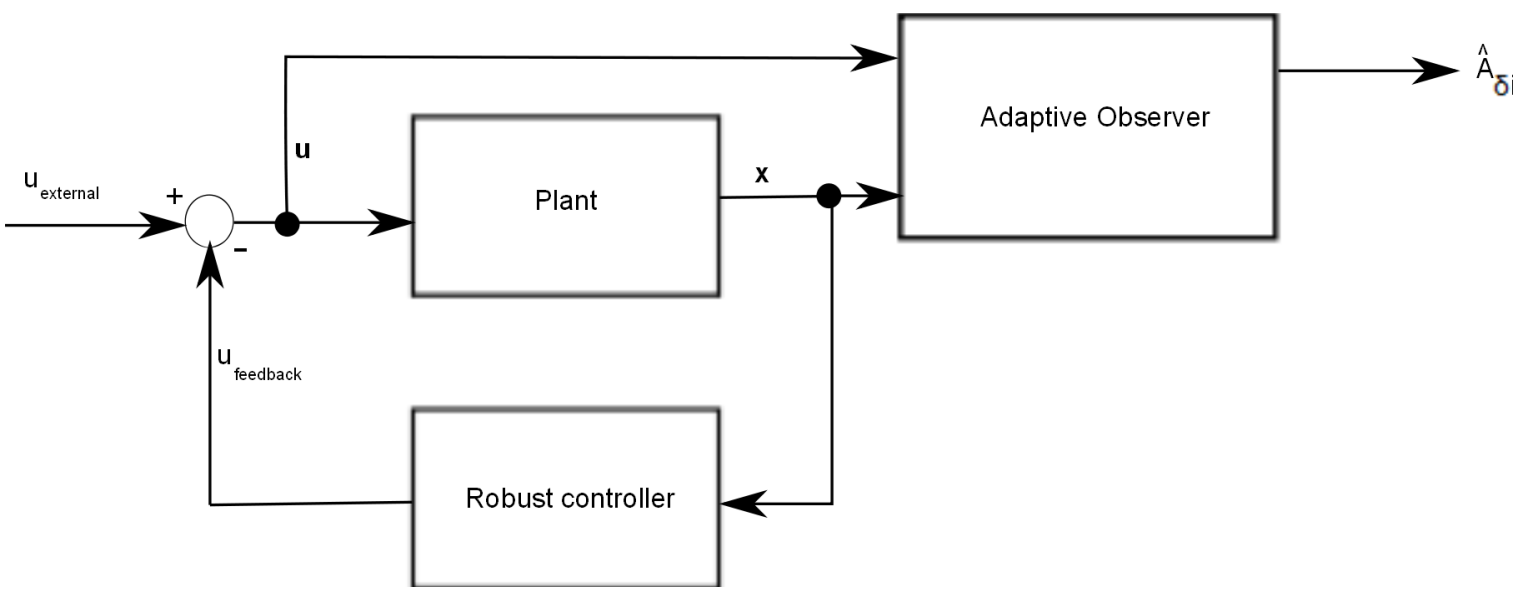

Figure 6-2: Parallel operation of adaptive observer and robust controller.

We consider the results from Case 3, Section 5-2 in Chapter 5. In addition, a robust controller designed for $\alpha=1, \zeta=1$, and $\phi=1$ is used to stabilize the system. The external input in Figure 5-1 is given to the system. Due to the presence of the controller, the actual input to the system is given in Figure 6-3.
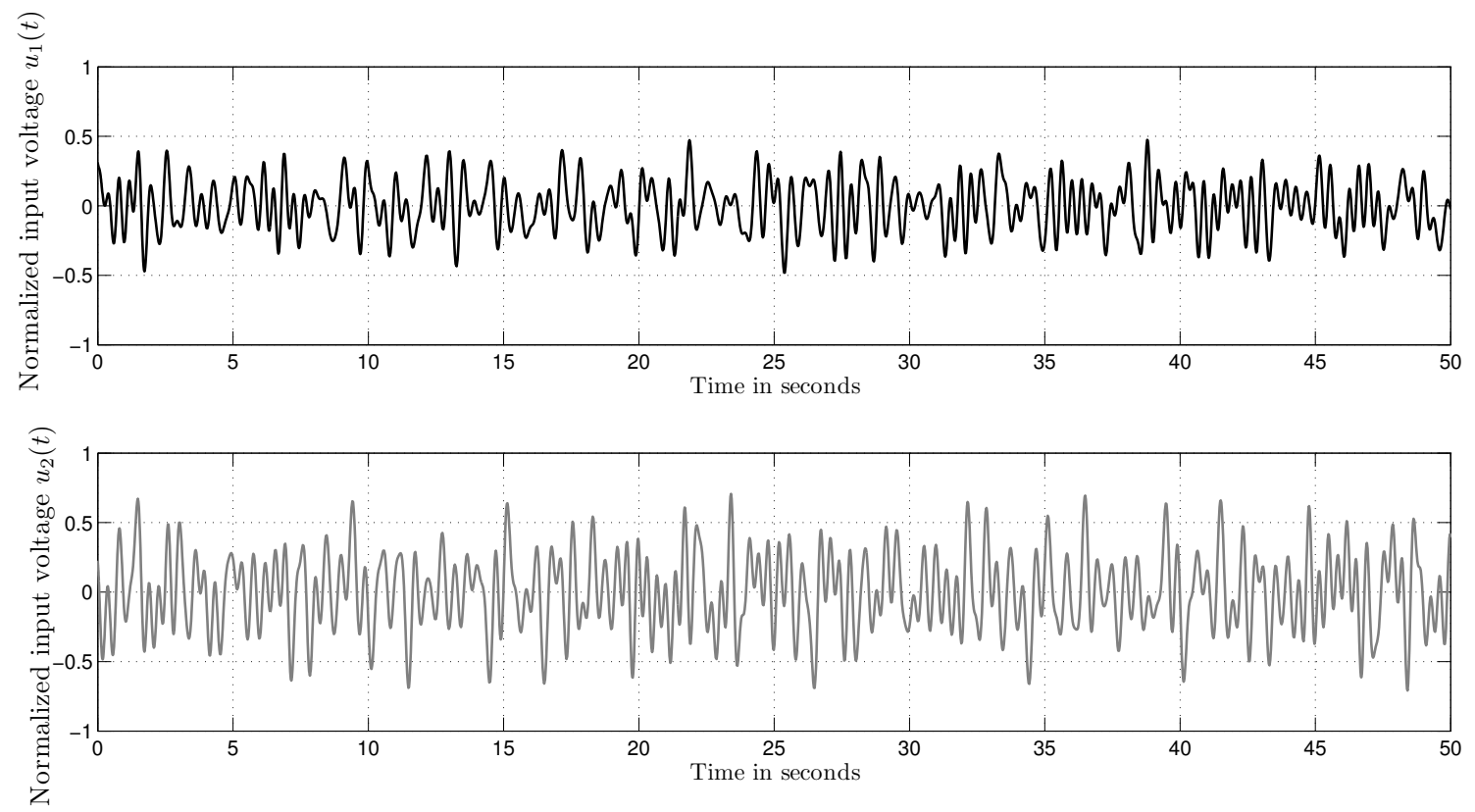

Figure 6-3: Input used to estimate uncertainty in the presence of controller.

Figures 6-4, 6-5, 6-7, and 6-6 present the activation of the rules, uncertainty estimation error, residual uncertainty norm and the state estimation error in the case of the uncertain TS fuzzy model being stabilized by the controller while the adaptive observer is operating in parallel. Simulation of the adaptive observer for $50 \mathrm{~s}$ with an active controller, resulted in the 

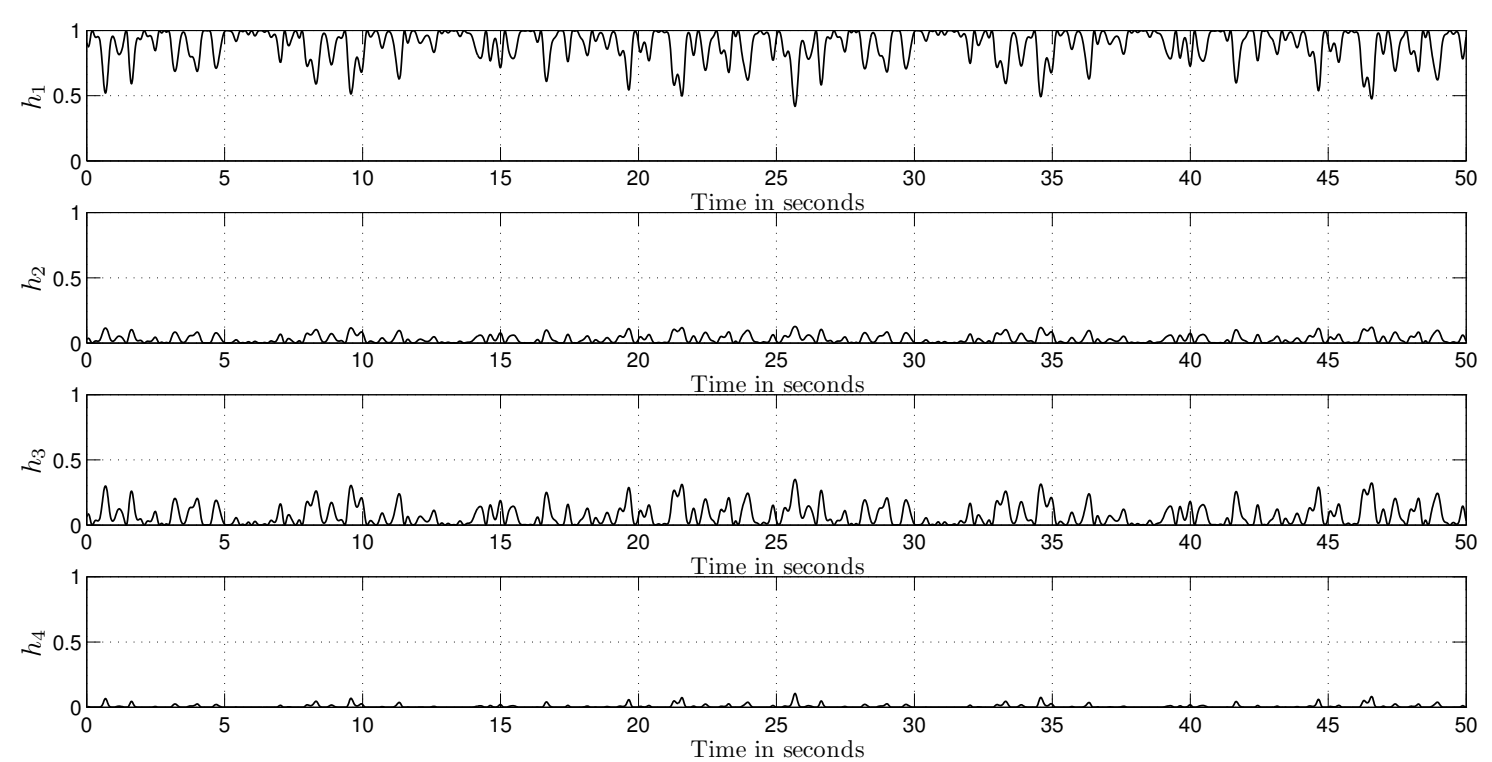

Figure 6-4: Activation of rules by the input used with an active controller.
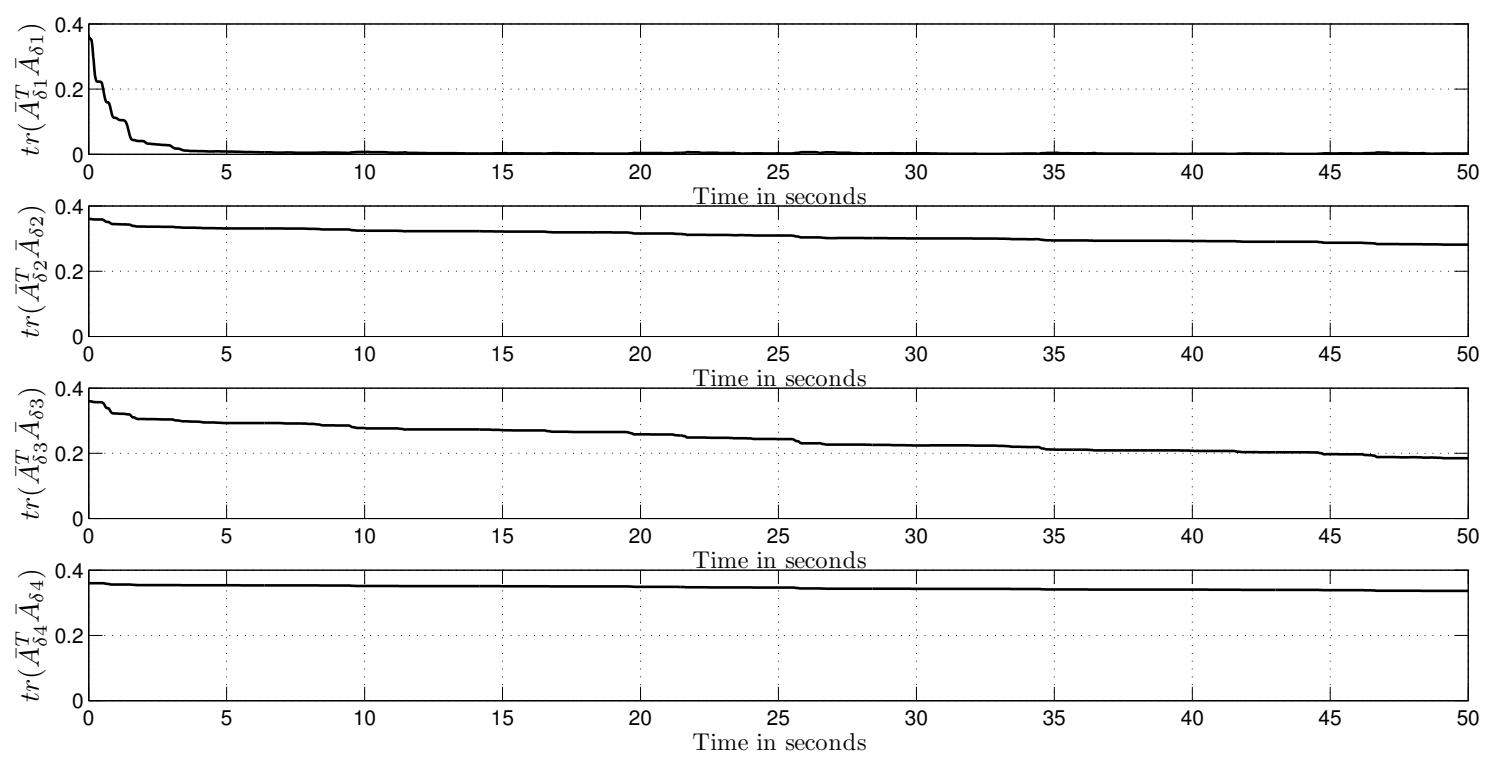

Figure 6-5: Uncertainty estimation error with uncertainty norm $=0.6, \mu_{\max }=200$, and an active controller.

uncertainty estimates:

$$
\begin{aligned}
& \widehat{A}_{\delta 1}=\left[\begin{array}{llll}
-0.0111 & 0.6448 & -0.0112 & 0.0122
\end{array}\right] \\
& \widehat{A}_{\delta 2}=\left[\begin{array}{llll}
0.0053 & 0.0696 & -0.0007 & 0.0002
\end{array}\right]
\end{aligned}
$$




$$
\begin{aligned}
& \widehat{A}_{\delta 3}=\left[\begin{array}{llll}
0.0130 & 0.1703 & -0.0017 & 0.0005
\end{array}\right] \\
& \widehat{A}_{\delta 4}=\left[\begin{array}{llll}
0.0018 & 0.0201 & -0.0002 & -0.0001
\end{array}\right]
\end{aligned}
$$

Comparing the results obtained in the Figure 6-5 with those obtained in Figure 5-9 Section
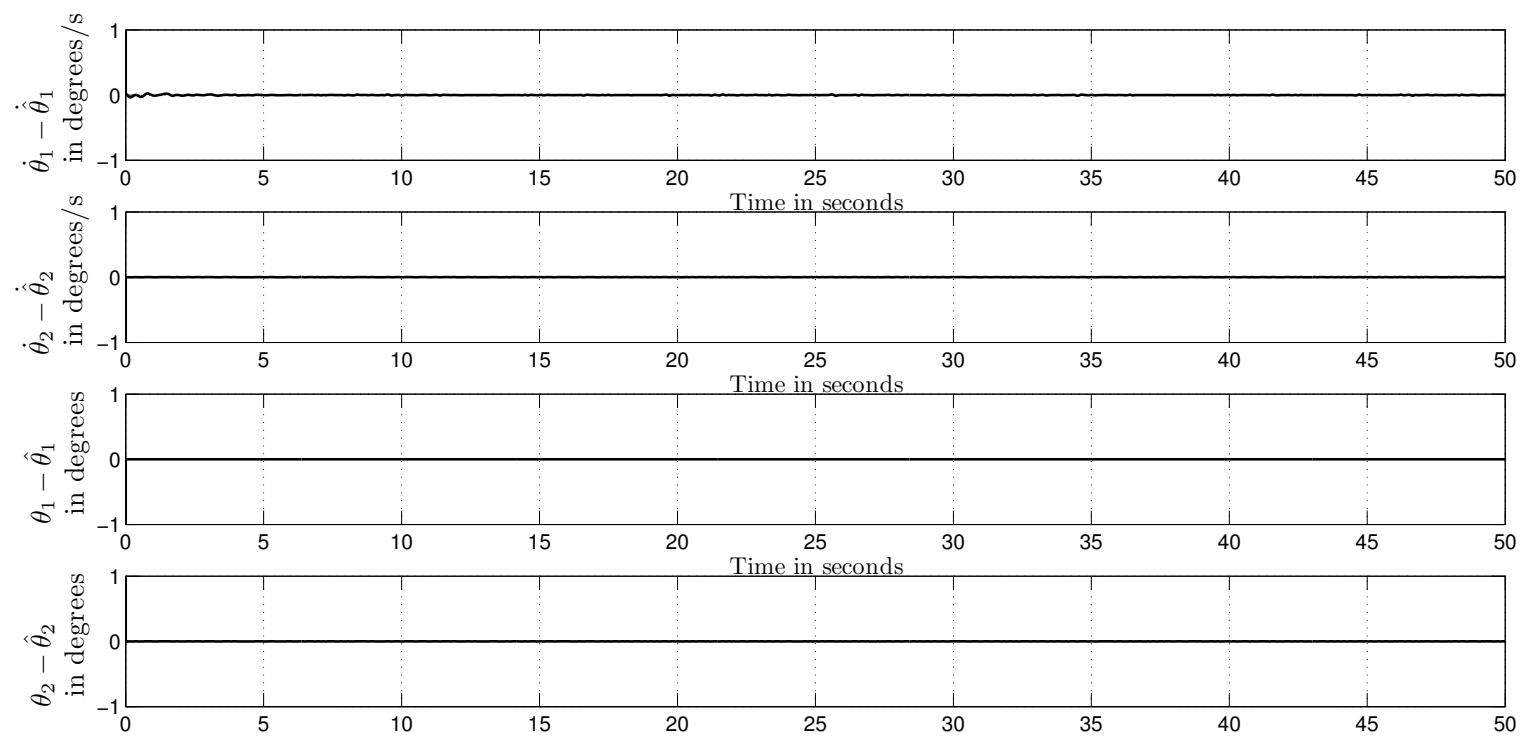

Figure 6-6: State estimation error with $\left\|A_{\delta i}\right\|=0.6, \mu_{\max }=200$, and an active controller.
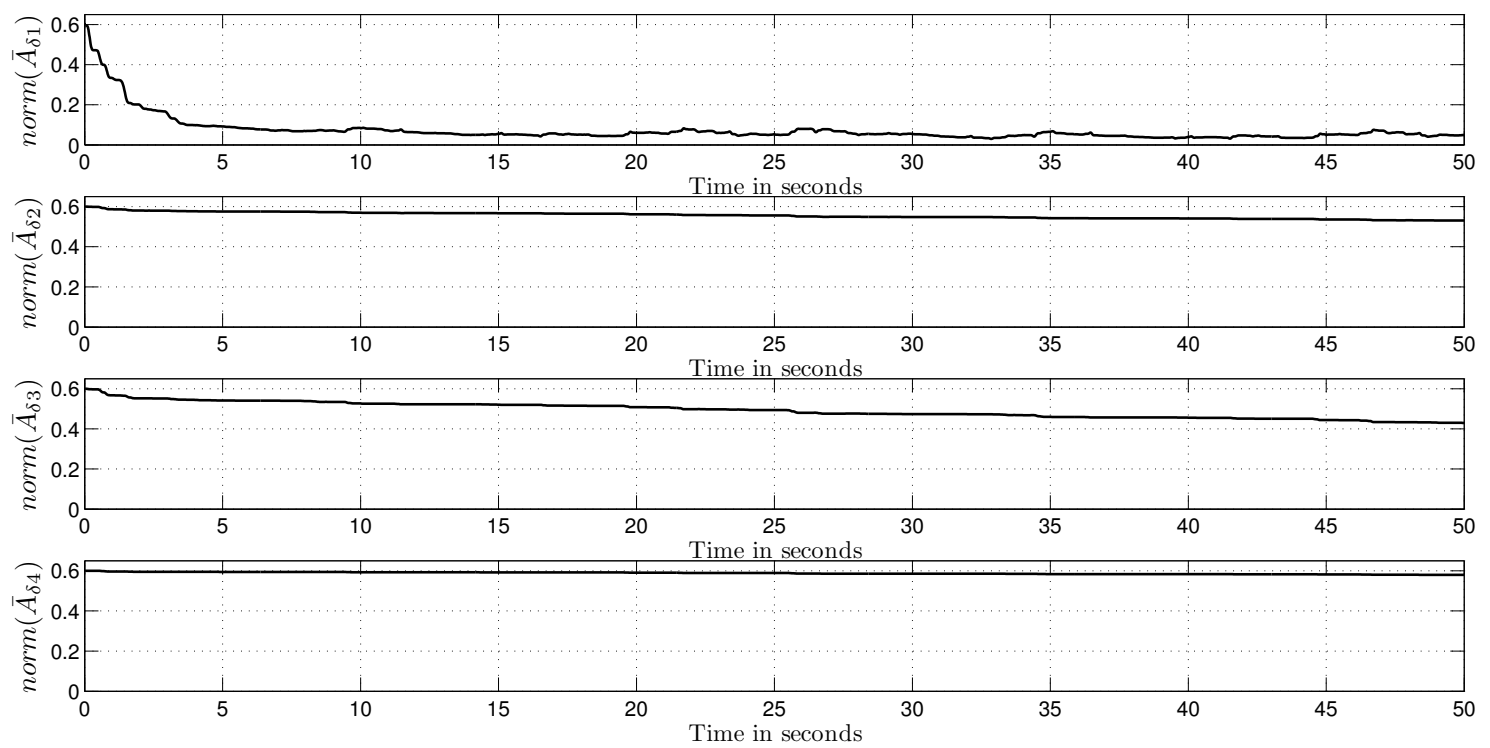

Figure 6-7: Norm of the residual uncertainty $\left\|A_{\delta i}\right\|=0.6, \mu_{\max }=200$ and an active controller.

5 -2, Case 3 , it is observed that the uncertainty estimation error after $50 \mathrm{~s}$ in case of estimation 
with active controller is higher than in case of stand-alone estimation by the adaptive observer. It can be seen from Figure 6-5 that the estimation is faster in case of rule $R_{1}$. This is due to the fact that the stabilizing action by the controller takes the states of the system towards zero. The value of $\theta_{2}$ closer to zero corresponds to higher activation of $R_{1}$. Thus it can be concluded that the stabilizing action reduces the uncertainty convergence rate in case of local models corresponding to $R_{2}, R_{3}$ and $R_{4}$.

\section{6-3 Conclusions}

In this chapter, the concepts of robust controller and adaptive observer discussed in Chapters 4 and 5 are brought together. The influence of using the uncertainty estimates from the adaptive observer in design of robust controller is analyzed. With an updated model of the system, it is found that the controller can guarantee stabilization with a higher decay rate.

The possibility to use the adaptive observer to estimate the uncertainty when a robust controller is stabilizing the system has been analyzed in analogy to closed loop identification. From the results, we found that the uncertainty convergence rate is reduced in the presence of the stabilizing controller for all the rules except the one that is activated more due to the stabilizing action.

The next chapter presents the conclusions derived from this thesis and proposals for future work. 


\section{Chapter 7}

\section{Conclusions and proposals for future work}

This thesis dealt with uncertainty estimation and control of nonlinear systems represented by Takagi-Sugeno (TS) fuzzy models. The TS models were assumed to have uncertainties in the state matrices of the local linear models. Adaptive observers introduced in the recent past were used to obtain estimates of uncertainties and update the model of the system. Stabilization and controller performance on using the updated model of the system was analyzed. To demonstrate the adaptive observer and the controller design, a 2-DOF robot arm model was used. The parameters of the robot arm were estimated for a laboratory-scale setup (Chapter 3). The nonlinear model of the robot arm, originally containing six nonlinearities was simplified to contain only two nonlinearities. Then a four-rule TS fuzzy model was constructed using the sector nonlinearity approach. The simplified nonlinear model and hence the fuzzy model almost exactly represent the complete nonlinear model. The mismatch in the plant and the nonlinear model was attributed to unmodelled dynamics in the state matrices. Assuming constant uncertainties in specific locations of the state matrices, an adaptive observer [1] was used to estimate them. The simulation results were presented in Chapter 5. The possibility to use the information about the structure of the uncertainties in the TS fuzzy model in designing the uncertainty estimation experiment was analyzed. The uncertainty estimates provided by the adaptive observer were used to update the fuzzy model of the nonlinear system. The new model was used in the design of robust state feedback stabilizing controller in Chapter 6. Since the estimates obtained from the adaptive observer were used in controller design, the uncertainty distribution structure used in the design of both adaptive observer and the robust controller needed to be same. Hence, a robust controller design was developed (Chapter 4) that uses the same uncertainty distribution structure as the adaptive observer. From the experimental results, it can be concluded that stability can be guaranteed with a higher decay rate when using the updated model in robust controller design (Chapter 6). The following paragraphs describe the work done and the conclusions derived in detail. 


\section{7-1 Conclusions}

- Trade-off between initial condition norm bound and maximum uncertainty norm bounds obtained by the robust controller design process

The robust controller design was demonstrated on the 2-DOF robot arm and results were presented in Chapter 4, Section 4-5. Two sets of results were presented with same performance specification (decay rate and control input constraint) but two different values of initial conditions of the system $\boldsymbol{x}(0)_{1}$ and $\boldsymbol{x}(0)_{2}$. It can be seen that with $\left\|\boldsymbol{x}(0)_{1}\right\|<\left\|\boldsymbol{x}(0)_{2}\right\|$, the maximum uncertainty bounds obtained by the controller design process are relatively smaller for the case with initial condition $\left\|\boldsymbol{x}(0)_{2}\right\|$. Hence, it can be concluded that a trade-off exists between the initial condition norm bound and the maximum uncertainty norm bounds obtained by the robust controller design process.

- Robust controller with an alternate uncertainty distribution structure

A robust controller was designed in Section 4-3, Chapter 4 considering uncertainty distribution structure $\Delta A_{i}=M_{i} A_{\delta i}$. Similar to the design procedure in [5], the uncertainties are bounded by a known norm and the maximum norm of the uncertainties for which the controller can guarantee stabilization can be obtained as part of the design process. Design specifications, namely decay rate and constraint on the control input are also included. Since the stability of the robust state feedback controller and adaptive observer with the uncertainty distribution structure $\Delta A_{i}=M_{i} A_{\delta i}$ are individually now proven, the stability analysis of a plant controlled by the robust controller with states estimated by the adaptive observer (along with uncertainty estimation) can follow.

- Robust controller design for unmodelled nonlinearities and time varying uncertainties

The simulation results in Section 4-5, Chapter 4 consider examples with constant uncertainties in the system matrices. However, the designed controller guarantees stability of the system as long as the uncertainty norm is smaller than the maximum uncertainty norm obtained as part of the controller design process. Hence, the controller design can as well be used in controlling other applications with unmodelled nonlinearities or time varying uncertainties.

\section{- Uncertainty distribution structures}

Compared to the uncertainty distribution structure $\Delta A_{i}=D_{a i} \Delta_{a i} E_{a i}$, the uncertainty distribution structure $\Delta A_{i}=M_{i} A_{\delta i}$ lacks the flexibility to concentrate uncertainty in a desired subsection of the state matrix.

- Higher uncertainty convergence rate with higher uncertainty norm bound in adaptive observer design 
The adaptive observer design was presented in Section 5-2, Chapter 5 for constant uncertainties in the state matrices of 2-DOF robot arm TS model. One of the inputs to the design is the value $\mu_{\max }$ which is the maximum uncertainty norm for which the adaptive observer should be designed (See [1]). The simulation results show that designing the adaptive observer for a much higher uncertainty norm bound than what is required by the system, a higher uncertainty convergence rate is achieved.

- Exploiting the structure of the TS model in designing uncertainty estimation experiments

In Section 5-3 of Chapter 5, the information that the nonlinearity/scheduling variable in the model depends only on the state variable $\theta_{2}$ was used in designing the estimation experiment. We also exploited the fact that the uncertainty is in a position that multiplies $\dot{\theta}_{2}$ and $u_{2}$ is sufficient to excite the system. In such a case, uncertainty estimation does not necessitate the design of input $u_{1}$ which is of practical advantage. This indicates that the structure of the nonlinearity and the uncertainty in the TS fuzzy model may simplify the uncertainty estimation experiment design.

- Guaranteed stability with higher decay rate for smaller uncertainty in the updated model

The uncertainty estimates obtained from the adaptive observer are used to update the model of the system and to design the robust controller in Section 6-1, Chapter 6. It can be concluded from the simulation results for the 2-DOF robot arm that with an updated model of the system, the controller can guarantee stabilization for a higher decay rate due to smaller uncertainties.

- Reduced uncertainty convergence rate in the presence of the stabilizing controller

Along the lines of closed loop identification, Section 6-2 of Chapter 6 presented the possibility to use an adaptive observer for the purpose of estimating uncertainties when a robust controller is actively stabilizing the system. The results show the reduction in the uncertainty convergence rate when the controller is active on the plant. However, it was observed that the uncertainty in case of the rule which corresponds to the states of the system being close to zero, the convergence rate is high.

- Conservativeness of the robust controller and adaptive observer

Design of both the robust controller and adaptive observer are based on common quadratic Lyapunov function. A common quadratic Lyapunov function requires all local models to be stable (for control purposes) and observable (for estimation purposes). A local model not being stable need not necessarily mean that the TS model in unstable throughout the statespace. It is possible that the global model is stable even when the local model is not (unless there is a possibility that the unstable local model can be the only once active). Hence the robust controller design and the adaptive observer design are conservative due to the use of common quadratic Lyapunov function. 
- Effect of equal and constant uncertainty assumption on TS fuzzy model

Assuming equal constant uncertainties attributed only to the variable $b_{2}$ is an important simplification considered in the thesis (see Section 5-2). An uncertainty in $b_{2}$ essentially causes uncertainties to appear in position $(2,2)$ of the state matrices (see Section 3-5) but is not considered in the thesis. However, the results obtained is expected to be similar in case of uncertainty in any position of the state matrices. The parameters $P_{1}, P_{2}$ and $P_{3}$ of the robot arm are found by nonlinear optimization (Section 3-3) using the values of $b_{1}, b_{2}, k_{m 1}$ and $k_{m 2}$ obtained by linear identification (Section 3-2). Hence, if we assume an uncertainty in the parameter $b_{2}$, since we have used $b_{2}$ in estimating $P_{1}, P_{2}$ and $P_{3}$, their estimates have high possibility of being uncertain. This essentially means the values of minimum and maximum values of the nonlinearities which are dependent on these parameters $P_{1}, P_{2}$ and $P_{3}$ are inexact as well (see equations (3-18), (3-19), and (3-34)). Due to time constraints, the effect of this inexactness on the fuzzy model is not considered in this thesis.

Directions for future work are presented in the following section.

\section{7-2 Future research proposals}

- A different approach to modelling of the robot arm

The simplified model and hence a TS model constructed from it have been shown to represent the complete nonlinear model almost exactly (see Figures 3-5 and 3-6). Hence for practical purposes, the simplified nonlinear model in equation (3-13) can be used to describe the robot arm. To estimate the parameters of the robot arm, an alternate approach can be taken. The exact values of the masses and lengths of the individual links can be measured before combining them to form the arm or obtained from the manufacturer of the arm. Subsequently the centre of masses and inertias of the individual links can be evaluated. From this exercise, parameters $P_{1}, P_{2}$ and $P_{3}$ present in the mass matrix of (3-13) can be found almost exactly. Modelling of the DC motor at the individual links can then be performed to obtain initial estimates of damping and motor gain of the nonlinear model. The uncertainty in the values of $b_{1}, b_{2}, k_{m 1}$, and $k_{m 2}$ in (3-15) can then be represented by constant uncertainties in the state matrices and input matrices. The adaptive observer for uncertainties both in the state matrices and input matrices [1] can be designed to estimate the uncertainties in the model.

- Maximizing the decay rate and uncertainty norms bounds

In the design of the robust controller in Chapter 4, the decay rate is provided by the user and the maximum bounds on the uncertainties are obtained as part of the design process. As an extension, further work can be done to analyze the possibility of maximizing both the decay rate and the bound on the uncertainties and obtaining them as part of the design process.

- Adaptive observer with uncertainty block $\Delta A_{i}=D_{a i} \Delta_{a i} E_{a i}$ 
The adaptive observer used in estimating uncertainty in Chapter 5 considers uncertainty distribution structure of the form $\Delta A_{i}=M_{i} A_{\delta i}$. The uncertainty distribution structure of the form $\Delta A_{i}=D_{a i} \Delta_{a i} E_{a i}$ as used in robust controller design in [5] is able to concentrate the uncertainty to desired subsection of the state matrix. With this advantage in consideration, the possibility to develop an adaptive observer design with uncertainty block $\Delta A_{i}=D_{a i} \Delta_{a i} E_{a i}$ can be investigated.

- Adaptive observers for other applications

The simulation results from Chapter 5 indicate the behaviour of the maximum uncertainty norm for which the adaptive observer is designed i.e., $\mu_{\max }$ to be an uncertainty convergence rate. These results are obtained specifically in case of the 2-DOF robot arm example. Designing the adaptive observer for various applications and testing with different values of uncertainty norm bounds is required to confirm these results from a general perspective. A rigorous mathematical analysis in this direction can be performed as well.

For the purpose of simulations, it is considered that the uncertainties in the state matrices are constant and that they have the same value in a specific position in the state matrices of all the local linear models. However, these constant uncertainties may have different values in different local linear models and may be present in different/multiple positions. Further work can be done to test the performance of the adaptive observer in case of constant but different/multiple uncertainties.

- State and uncertainty estimation

In the example in this thesis, the states are all considered to be measured. However, in practice this may not be true. Hence examples in which the control law depends on the states estimated by the adaptive observer (along with estimating uncertainties) need to be analyzed. A mathematical proof of stabilization of the plant while being controlled by a robust controller that uses states estimated by an adaptive observer will be necessary.

- Experiment design

The input considered in Chapter 5 is based on the knowledge of the bandwidth of the system. The adaptive observer requires sufficient activation of all the rules of the TS fuzzy model to enable efficient uncertainty estimation. Creating inputs to the system which ensure sufficient activation of all the rules, though seems application dependent, should be investigated. The duration for which the uncertainty estimation is required to be done to achieve sufficient improvement in the model is also a research area of practical implication.

- Saturation-like nonlinearities

In practical applications, the state variables have maximum limits due to actuator limitations or their physical structure. These limits can be incorporated into the fuzzy models as part of the definition of compact state space. However, the effect of these nonlinearities on the performance of adaptive observers is an area that needs to be investigated. 
- Discrete time analysis

The design of robust controller and adaptive observer for the 2-DOF robot arm have been done using continuous time TS systems. Discrete time analysis and implementation can be considered in the future.

- Effect of noise

The robust controller and adaptive observer simulations have been done without considering the effect of noise. Implementations with noise should also be analyzed. 


\section{Appendix A}

\section{Sector nonlinearity approach}

This appendix presents the sector nonlinearity approach that is used in constructing the Takagi-Sugeno (TS) fuzzy models for the application examples in Appendix B. The concepts in this appendix are adapted from [22], [5] and [1].

The sector nonlinearity approach provides an exact representation of a nonlinear system in a compact set of the state space [5]. For a simple nonlinear system with a single variable $\dot{x}(t)=f(x(t))$ given $f(0)=0$, the sector nonlinearity aims at finding a sector such that $\dot{x}(t)=f(x(t)) \in\left[a_{1}, a_{2}\right] x(t)$. It exactly represents the nonlinear system $f(x(t))$ with the sector bounded by the straight lines $a_{1} x(t)$ and $a_{2} x(t)$. The idea is illustrated by Figure A-1 taken from [22]. From the slopes $a_{1}, a_{2}$ of the straight lines and the function $f(x(t))$,

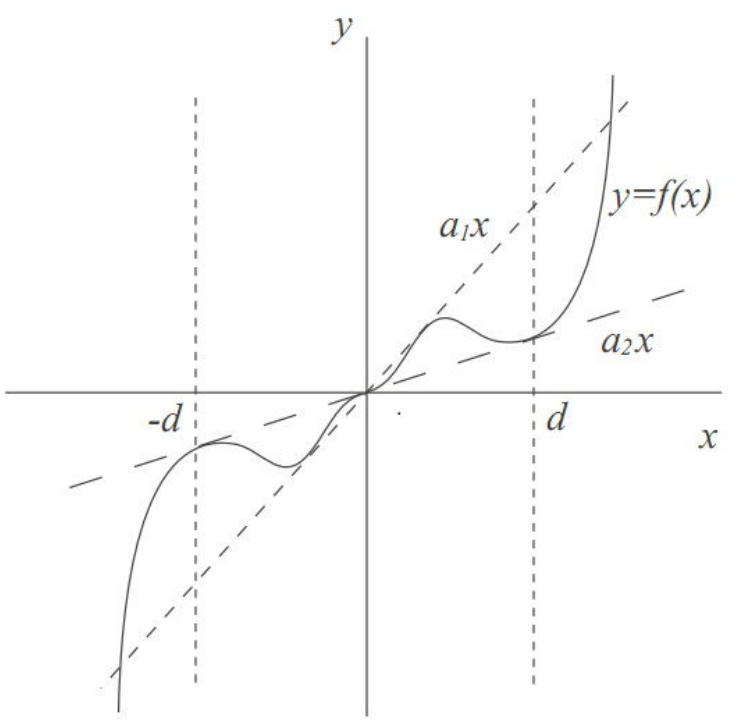

Figure A-1: Local sector $\in[-d, d]$ 
membership functions are created as follows.

$$
\begin{aligned}
& h_{1}(x)=\frac{f(x(t))-a_{2} x(t)}{\left(a_{1}-a_{2}\right) x(t)} \\
& h_{2}(x)=\frac{a_{1} x(t)-f(x(t))}{\left(a_{1}-a_{2}\right) x(t)}
\end{aligned}
$$

where $h_{1}(x)+h_{2}(x)=1$ and $h_{1}(x), h_{2}(x) \geq 0$.

From the membership functions, the nonlinear function can be reconstructed by

$$
\begin{aligned}
f(x(t)) & =h_{1}(x) a_{1} x(t)+h_{2}(x) a_{2} x(t) \\
& =\sum_{i=1}^{2} h_{i}(x) a_{i} x(t)
\end{aligned}
$$

Associating the membership functions $h_{1}(x)$ and $h_{2}(x)$ with the fuzzy sets $N_{1}^{1}$ and $N_{2}^{1}$ and the scheduling variable $z_{1}(t)=f(x(t))$, we have the following two rules representing this nonlinearity.

$$
\begin{gathered}
R_{1}: \text { If } z_{1} \text { is } N_{1}^{1} \text { then } \\
f(x(t))=a_{1} x(t) \\
R_{2}: \text { If } z_{1} \text { is } N_{2}^{1} \text { then } \\
f(x(t))=a_{2} x(t)
\end{gathered}
$$

Figure A-1 represents the concept of sector nonlinearity, where the nonlinearity is covered in a defined range of $x(t)$.

In the following, sector nonlinearity is illustrated on two numerical examples. The first example is from [5] and the second example is similar to those in [1].

Example 1. For a nonlinear system

$$
f(x(t))=\sin (x(t))
$$

with $x(t) \in\left[-\frac{\pi}{2}, \frac{\pi}{2}\right]$, a sector bounded by two lines $a_{1} x(t)$ and $a_{2} x(t)$ ( $a_{1}$ and $a_{2}$ are slopes) can be found. Considering a new variable $z_{1}(t)=\sin (x(t))$ and the slopes $a_{1}=1$ and $a_{2}=\frac{2}{\pi}$ (represented in the Figure A-2), we can represent the nonlinearity in equation (A-4) as

$$
z_{1}=\sin (x(t))=h_{1}\left(z_{1}\right) a_{1} x(t)+h_{2}\left(z_{1}\right) a_{2} x(t)
$$

where

$$
\begin{aligned}
& h_{1}\left(z_{1}\right)=\left\{\begin{array}{lr}
\frac{\sin (x(t))-a_{2} x(t)}{\left(a_{1}-a_{2}\right) x(t)}, & x(t) \neq 0, \\
1, & \text { otherwise }
\end{array}\right. \\
& h_{2}\left(z_{1}\right)=\left\{\begin{array}{lr}
\frac{a_{1} x(t)-\sin (x(t))}{\left(a_{1}-a_{2}\right) x(t)}, & x(t) \neq 0, \\
0, & \text { otherwise }
\end{array}\right.
\end{aligned}
$$


i.e.,

$$
\begin{aligned}
& h_{1}\left(z_{1}\right)=\left\{\begin{array}{lr}
\frac{z_{1}-a_{2} x(t)}{\left(a_{1}-a_{2}\right) x(t)}, & x(t) \neq 0, \\
1, & \text { otherwise }
\end{array}\right. \\
& h_{2}\left(z_{1}\right)=\left\{\begin{array}{lc}
\frac{a_{1} x(t)-z_{1}}{\left(a_{1}-a_{2}\right) x(t)}, & x(t) \neq 0, \\
0, & \text { otherwise }
\end{array}\right.
\end{aligned}
$$

The TS fuzzy model representing nonlinear system (A-4) will be

$$
\begin{gathered}
R_{1}: \text { If } z_{1} \text { is } N_{1}^{1} \text { then } \\
f(x(t))=\frac{2}{\pi} x(t) \\
R_{2}: \text { If } z_{1} \text { is } N_{2}^{1} \text { then } \\
f(x(t))=x(t)
\end{gathered}
$$

which can be written as

$$
f(x(t))=h_{1}\left(z_{1}\right) a_{1} x(t)+h_{2}\left(z_{1}\right) a_{2} x(t)
$$

Thus the nonlinearity/nonlinear system $f(x(t))=\sin (x(t))$ is represented by a TS fuzzy model using sector nonlinearity.

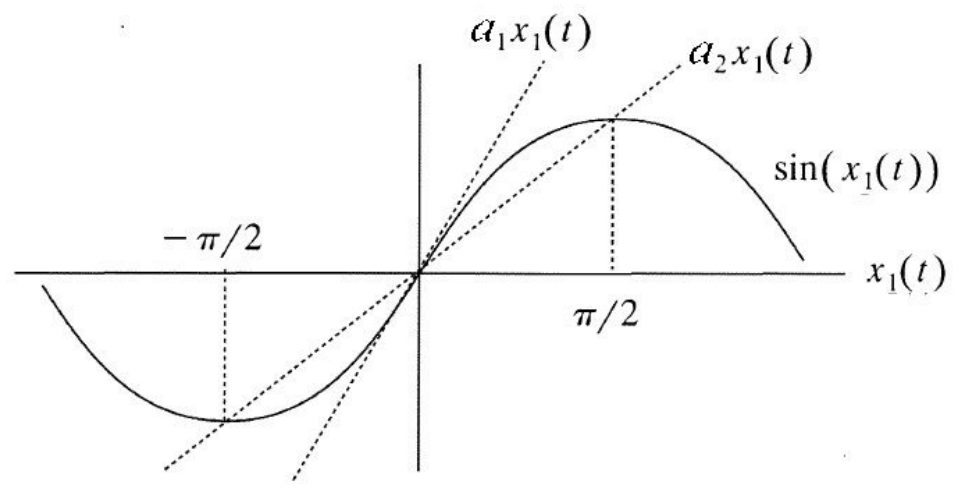

Figure A-2: Sector for $\sin (x(t)) \in\left[\frac{-\pi}{2}, \frac{\pi}{2}\right]$

Example 2. Consider a nonlinear system,

$$
\begin{aligned}
\dot{x}_{1} & =x_{1} x_{2}+u \\
\dot{x}_{2} & =x_{1}+x_{2}^{2} \\
y & =x_{1}+x_{2}
\end{aligned}
$$

The above nonlinear system can be represented as

$$
\begin{aligned}
\dot{\boldsymbol{x}} & =A \boldsymbol{x}+B u \\
y & =C \boldsymbol{x}
\end{aligned}
$$


where

$$
\begin{aligned}
A & =\left[\begin{array}{cc}
x_{2} & 0 \\
1 & x_{2}
\end{array}\right] \\
B & =\left[\begin{array}{l}
1 \\
0
\end{array}\right] \\
C & =\left[\begin{array}{ll}
1 & 1
\end{array}\right]
\end{aligned}
$$

Given $x_{1} \in[-1,1]$ and $x_{2} \in[-1,1]$ and considering the scheduling variable $z_{1}=x_{2}$ (it is a non-constant term in the state matrix and is a part of the nonlinear terms $x_{1} x_{2}$ and $x_{2}^{2}$ ), we can represent $z_{1}$ as

$$
z_{1}=x_{2}=h_{1}\left(z_{1}\right) a_{1}+h_{2}\left(z_{1}\right) a_{2}
$$

where

$$
\begin{aligned}
& h_{1}\left(z_{1}\right)=\frac{z_{1}+1}{1+1} \\
& h_{2}\left(z_{1}\right)=\frac{1-z_{1}}{1+1}
\end{aligned}
$$

and $a_{1}, a_{2}$ are the maximum and minimum values of the nonlinearity $z_{1}$, which thus allows $x_{1} x_{2} \in\left[a_{1}, a_{2}\right] x_{1}$ and $x_{2}^{2} \in\left[a_{1}, a_{2}\right] x_{2}$.

Associating membership functions $h_{1}\left(z_{1}\right)$ and $h_{2}\left(z_{1}\right)$ with fuzzy sets $N_{1}^{1}$ and $N_{2}^{1}$, the nonlinear system (A-7) can be written as a TS fuzzy system as follows.

$$
\begin{aligned}
& R_{1}: \text { If } z_{1} \text { is } N_{1}^{1} \text { then } \\
& \dot{\boldsymbol{x}}=\left[\begin{array}{ll}
1 & 0 \\
1 & 1
\end{array}\right] \boldsymbol{x}+\left[\begin{array}{l}
1 \\
0
\end{array}\right] u \\
& y=\left[\begin{array}{ll}
1 & 1
\end{array}\right] \boldsymbol{x} \\
& R_{2}: \text { If } z_{1} \text { is } N_{2}^{1} \text { then } \\
& \dot{\boldsymbol{x}}=\left[\begin{array}{cc}
-1 & 0 \\
1 & -1
\end{array}\right] \boldsymbol{x}+\left[\begin{array}{l}
1 \\
0
\end{array}\right] u \\
& y=\left[\begin{array}{ll}
1 & 1
\end{array}\right] \boldsymbol{x}
\end{aligned}
$$

In case of nonlinear system with several nonlinearities, the procedure as explained above can be applied to every nonlinearity. Since every nonlinearity results in two membership functions, a fuzzy model represented by these nonlinearities will have the number of rules equal to $2^{p}$ where $p$ is the number of nonlinearities. 


\section{Appendix B}

\section{Application examples}

This appendix presents the construction of Takagi-Sugeno (TS) fuzzy models for three nonlinear systems using sector nonlinearity approach [5]. The three systems considered are:

- a 2-DOF helicopter model considering movement about the pitch axis

- the Delft robot arm

- a 2-DOF robot arm working in the horizontal plane

\section{B-1 2-DOF helicopter model}

The 2-DOF helicopter model is shown in Figure B-1. Based on first principles modelling, the

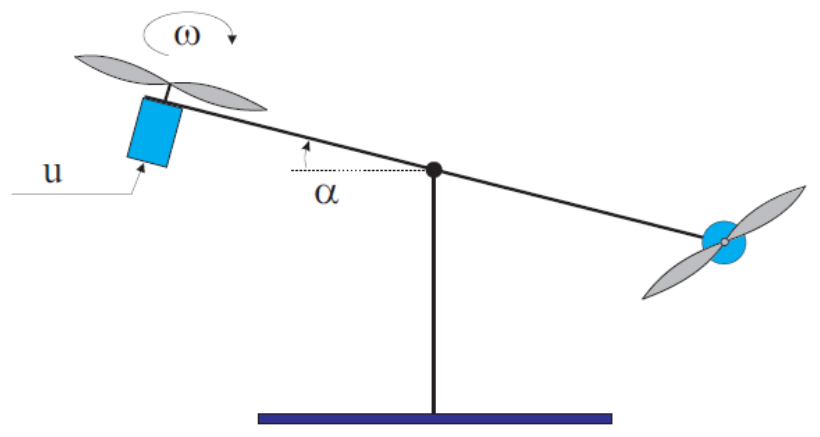

Figure B-1: 2-DOF laboratory scale helicopter

mathematical description of the pitch of the 2-DOF helicopter is

$$
\begin{aligned}
\tau \dot{\omega}+\omega & =K_{1} u \\
\ddot{\alpha}+b \dot{\alpha}+K_{2} \sin \alpha & =f(\omega)
\end{aligned}
$$

where 
- $\alpha$ is the pitch angle.

- $\omega$ is the speed of the propeller.

- $f(\omega)$ is the force exerted by the propeller considered as a function of the propeller speed.

- $K_{1}, K_{2}, b$ are constants ( $K_{1}$ is the gain from the control signal to the propeller's velocity, $b$ is the damping of the helicopter beam and $K_{2}$ is a constant related to influence of gravity).

- $\tau$ is the time constant of the propeller motor.

- $u$ is the input voltage applied to the propeller.

The relation between the speed of the propeller $\omega$ and the force applied by the propeller $f(\omega)$ is nonlinear. By approximating the relation between the force and the propeller speed as

$$
f(\omega)=K_{3} \omega
$$

the model of the system can be represented as

$$
\begin{aligned}
\dot{\omega}+\frac{\omega}{\tau} & =\frac{K_{1}}{\tau} u \\
\ddot{\alpha}+b \dot{\alpha}+K_{2} \sin \alpha & =K_{3} \omega
\end{aligned}
$$

Considering the states of the system as $\boldsymbol{x}=\left[\begin{array}{lll}\omega & \dot{\alpha} & \alpha\end{array}\right]^{\prime}$, the nonlinear system can be represented in a state space form.

$$
\left[\begin{array}{c}
\dot{\omega} \\
\ddot{\alpha} \\
\dot{\alpha}
\end{array}\right]=\left[\begin{array}{ccc}
\frac{-1}{\tau} & 0 & 0 \\
K_{3} & -b & 0 \\
0 & 1 & 0
\end{array}\right]\left[\begin{array}{c}
\omega \\
\dot{\alpha} \\
\alpha
\end{array}\right]+\left[\begin{array}{c}
\frac{K_{1}}{\tau} \\
0 \\
0
\end{array}\right] u+\left[\begin{array}{c}
0 \\
-K_{2} \sin \alpha \\
0
\end{array}\right]
$$

Considering a scheduling variable $z_{1}=\sin \alpha$ and $\alpha \in\left[\frac{-\pi}{6}, \frac{\pi}{6}\right]$, a sector $\left[b_{1}, b_{2}\right] \alpha$ can be found such that $\sin \alpha \in\left[b_{1}, b_{2}\right] \alpha$. If $\alpha \in\left[\frac{-\pi}{6}, \frac{\pi}{6}\right]$, then $\left[b_{1}, b_{2}\right]=\left[\frac{\sin \frac{\pi}{6}}{\frac{\pi}{6}}, \frac{2}{\pi}\right]$, see [5] and Appendix A. By using the sector nonlinearity approach for the nonlinearity $z_{1}$ as explained in Appendix A,

$$
z_{1}=\sin \alpha=h_{1}\left(z_{1}\right) b_{1} \alpha+h_{2}\left(z_{1}\right) b_{2} \alpha
$$

where

$$
h_{1}\left(z_{1}\right)+h_{2}\left(z_{1}\right)=1 \text { and } h_{1}\left(z_{1}\right), h_{2}\left(z_{1}\right) \geq 0
$$

and $h_{1}\left(z_{1}\right), h_{2}\left(z_{1}\right)$ are the membership functions. $h_{1}\left(z_{1}\right)$ and $h_{2}\left(z_{1}\right)$ are

$$
\begin{aligned}
& h_{1}\left(z_{1}\right)= \begin{cases}\frac{\sin \alpha-b_{2} \alpha}{\left(b_{1}-b_{2}\right) \alpha}, & \text { when } \alpha \neq 0 \\
1, & \text { when } \alpha=0\end{cases} \\
& h_{2}\left(z_{1}\right)= \begin{cases}\frac{b_{1} \alpha-\sin \alpha}{\left(b_{1}-b_{2}\right) \alpha}, & \text { when } \alpha \neq 0 \\
0, & \text { when } \alpha=0\end{cases}
\end{aligned}
$$


i.e.,

$$
\begin{aligned}
& h_{1}\left(z_{1}\right)= \begin{cases}\frac{z_{1}-b_{2} \alpha}{\left(b_{1}-b_{2}\right) \alpha}, & \text { when } \alpha \neq 0 \\
1, & \text { when } \alpha=0\end{cases} \\
& h_{2}\left(z_{1}\right)= \begin{cases}\frac{b_{1} \alpha-z_{1}}{\left(b_{1}-b_{2}\right) \alpha}, & \text { when } \alpha \neq 0 \\
0, & \text { when } \alpha=0\end{cases}
\end{aligned}
$$

Associating the membership functions $h_{1}\left(z_{1}\right)$ and $h_{2}\left(z_{1}\right)$ with fuzzy sets $N_{1}^{1}$ and $N_{2}^{1}$, we have the fuzzy model of the system represented by (B-1) with 2 rules as follows.

$$
\begin{aligned}
& R_{i}: \text { If } z_{1} \text { is } N_{i} \text { then } \\
& \begin{aligned}
\dot{\boldsymbol{x}} & =A_{i} \boldsymbol{x}+B_{i} u \\
\boldsymbol{y} & =C_{i} \boldsymbol{x}
\end{aligned}
\end{aligned}
$$

for $i=1,2$, where

$$
\begin{aligned}
& \boldsymbol{x}=\left[\begin{array}{ccc}
\omega & \dot{\alpha} & \alpha
\end{array}\right]^{T} \\
& A_{1}=\left[\begin{array}{ccc}
\frac{-1}{\tau} & 0 & 0 \\
K_{3} & -b & -K_{2} b_{1} \\
0 & 1 & 0
\end{array}\right] \quad A_{2}=\left[\begin{array}{ccc}
\frac{-1}{\tau} & 0 & 0 \\
K_{3} & -b & -K_{2} b_{2} \\
0 & 1 & 0
\end{array}\right] \\
& B_{1}=B_{2}=\left[\begin{array}{c}
\frac{K_{1}}{\tau} \\
0 \\
0
\end{array}\right] \quad C_{1}=C_{2}=\left[\begin{array}{lll}
1 & 0 & 0 \\
0 & 0 & 1
\end{array}\right] .
\end{aligned}
$$

This completes the construction of the TS fuzzy model for the pitch of the 2-DOF helicopter model. If there exists uncertainty in the damping of the beam $b$, then the model can be considered as an uncertain TS fuzzy model with uncertainty in the state matrix.

\section{B-2 Delft robot arm}

The Delft robot arm is presented in Figure B-2.

The model of this nonlinear system is

$$
\begin{aligned}
m L^{2} \ddot{\phi}+(K u r-m g L) \sin \phi & =\tau \\
k u-K r \cos \phi & =F
\end{aligned}
$$

where

- $\phi$ is the angle of the robot arm.

- $\dot{\phi}$ is the angular velocity of the robot arm. 


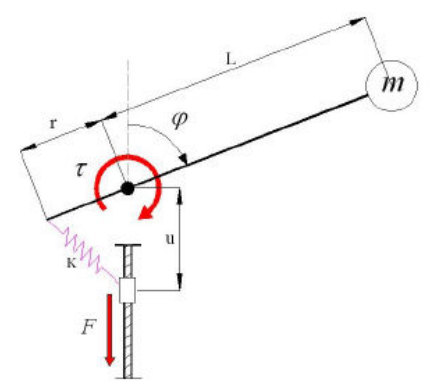

Figure B-2: Delft robot arm

- $g$ is the acceleration due to gravity.

- $K$ is the spring constant.

- $L$ is the length of the link.

- $r$ is the length of the spring arm.

- $\tau$ is the driving torque and $u$ is the input to the system given in terms of distance.

Considering that the arm is operated by using only the input $u$ (the torque input $\tau=0$ ), the state space model of the system is

$$
\begin{aligned}
\dot{x_{1}} & =x_{2} \\
\dot{x_{2}} & =\sin \left(x_{1}\right) \frac{g}{L}-\sin \left(x_{1}\right) \frac{K r}{L^{2} m} u \\
y & =x_{1}
\end{aligned}
$$

where $x_{1}=\phi$ is the angular position of the robot arm and is measured.

Considering $\boldsymbol{x}=\left[\begin{array}{ll}x_{1} & x_{2}\end{array}\right]^{T}$, we have

$$
\dot{\boldsymbol{x}}=\left[\begin{array}{ll}
0 & 1 \\
0 & 0
\end{array}\right] \boldsymbol{x}+\left[\begin{array}{c}
0 \\
-\sin \left(x_{1}\right) \frac{K r}{L^{2} m}
\end{array}\right] u+\left[\begin{array}{c}
0 \\
\sin \left(x_{1}\right) \frac{g}{L}
\end{array}\right]
$$

Applying sector nonlinearity to the two nonlinear terms $z_{1}=\sin \left(x_{1}\right)$ in the input matrix and $z_{2}=\sin \left(x_{1}\right)$ in the affine matrix, we can convert the above model to a TS fuzzy model. The nonlinearity in the affine term $z_{1}=\sin \left(x_{1}\right)$ is bounded by $\left[p_{1}, p_{2}\right] x_{1}$, given that $x_{1}$ changes between $[\pi / 12, \pi / 2], p_{1} x \leq \sin \left(x_{1}\right) \leq p_{2} x$. The value of $p_{1}$ and $p_{2}$ are found to be $p_{1}=\frac{\sin \frac{\pi}{12}}{\frac{\pi}{12}}$ and $p_{2}=\frac{2}{\pi}$. The other nonlinear term $z_{2}=\sin \left(x_{1}\right)$ in the input matrix is bounded by $\left[z_{2 \min }\right.$, $\left.z_{2 \max }\right]$ where $z_{2 \min }=\sin (\pi / 12)=0.2588$ and $z_{2 \max }=\sin (\pi / 2)=1$. Hence we have,

$$
\begin{aligned}
& z_{1}=w_{11} p_{1} x_{1}+w_{12} p_{2} x_{1} \\
& z_{2}=w_{21} z_{2 \min }+w_{22} z_{2 \max }
\end{aligned}
$$


where $w_{11}+w_{12}=1$ and $w_{21}+w_{22}=1$

$$
\begin{aligned}
& w_{11}=\left\{\begin{array}{lr}
\frac{\sin (x(t))-p_{2} x(t)}{\left(p_{1}-p_{2}\right) x(t)}, & x(t) \neq 0, \\
1, & \text { otherwise }
\end{array}\right. \\
& w_{12}=\left\{\begin{array}{lr}
\frac{p_{1} x(t)-\sin (x(t))}{\left(p_{1}-p_{2}\right) x(t)}, & x(t) \neq 0, \\
0, & \text { otherwise }
\end{array}\right. \\
& w_{21}=\frac{z_{2 \max }-z_{2}}{z_{2 \max }-z_{2 \min }} \\
& w_{22}=\frac{z_{2}-z_{2 \min }}{z_{2 \max }-z_{2 \min }}
\end{aligned}
$$

Since we have two nonlinearities and each nonlinearity is associated with 2 rules, the entire nonlinear system can be represented by 4 rules. Associating membership functions $w_{11}, w_{12}$, $w_{21}$, and $w_{22}$ with fuzzy sets $N_{1}^{1}, N_{2}^{1}, N_{1}^{2}$, and $N_{2}^{2}$ respectively, we have

$R_{1}$ : If $z_{1}$ is $N_{1}^{1}$ and $z_{2}$ is $N_{1}^{2}$ then

$$
\begin{aligned}
\dot{\boldsymbol{x}} & =\left[\begin{array}{cc}
0 & 1 \\
\frac{p_{1} g}{L} & 0
\end{array}\right] \boldsymbol{x}+\left[\begin{array}{c}
0 \\
\frac{z_{2 \min K r}}{L^{2} m}
\end{array}\right] u \\
\boldsymbol{y} & =\left[\begin{array}{ll}
1 & 0
\end{array}\right] \boldsymbol{x}
\end{aligned}
$$

$R_{2}:$ If $z_{1}$ is $N_{2}^{1}$ and $z_{2}$ is $N_{2}^{2}$ then

$$
\begin{aligned}
\dot{\boldsymbol{x}} & =\left[\begin{array}{cc}
0 & 1 \\
\frac{p_{2} g}{L} & 0
\end{array}\right] \boldsymbol{x}+\left[\begin{array}{c}
0 \\
\frac{z_{2 \min K r}}{L^{2} m}
\end{array}\right] u \\
\boldsymbol{y} & =\left[\begin{array}{ll}
1 & 0
\end{array}\right] \boldsymbol{x}
\end{aligned}
$$

$R_{3}:$ If $z_{1}$ is $N_{3}^{1}$ and $z_{2}$ is $N_{3}^{2}$ then

$$
\begin{aligned}
\dot{\boldsymbol{x}} & =\left[\begin{array}{cc}
0 & 1 \\
\frac{p_{1} g}{L} & 0
\end{array}\right] \boldsymbol{x}+\left[\begin{array}{c}
0 \\
\frac{z_{2 \max } K r}{L^{2} m}
\end{array}\right] u \\
\boldsymbol{y} & =\left[\begin{array}{ll}
1 & 0
\end{array}\right] \boldsymbol{x}
\end{aligned}
$$

$R_{4}:$ If $z_{1}$ is $N_{4}^{1}$ and $z_{2}$ is $N_{4}^{2}$ then

$$
\begin{aligned}
\dot{\boldsymbol{x}} & =\left[\begin{array}{cc}
0 & 1 \\
\frac{p_{2} g}{L} & 0
\end{array}\right] \boldsymbol{x}+\left[\begin{array}{c}
0 \\
\frac{z_{2 \max } K r}{L^{2} m}
\end{array}\right] u \\
\boldsymbol{y} & =\left[\begin{array}{ll}
1 & 0
\end{array}\right] \boldsymbol{x}
\end{aligned}
$$

where $N_{3}^{1}=N_{1}^{1}, N_{3}^{2}=N_{2}^{2}, N_{4}^{1}=N_{2}^{1}$, and $N_{4}^{2}=N_{2}^{2}$. This completes the construction of the TS fuzzy model for the Delft robot arm. If the exact value of mass $m$ held by the arm is not known, then this system can be considered an uncertain TS fuzzy model with uncertainty in the input matrix. 


\section{B-3 2-DOF robot arm}

The 2-DOF robot arm is presented in Figure B-3. The arm operates only in the horizontal plane. The arm consists of two joints each with a DC motor [23].

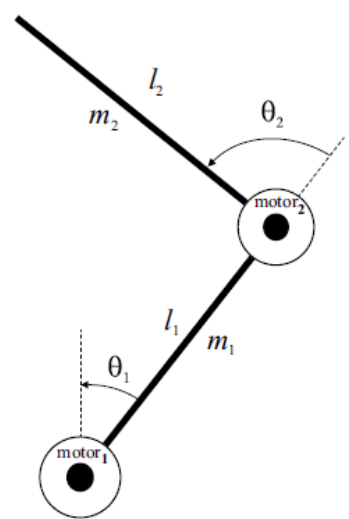

Figure B-3: Two link robot arm

The model of this nonlinear system is

$$
M(\theta) \ddot{\theta}+C(\theta, \dot{\theta}) \dot{\theta}+G(\theta)=\tau
$$

where

- $\theta=\left[\begin{array}{ll}\theta_{1} & \theta_{2}\end{array}\right]^{T}$ and $\tau=\left[\begin{array}{ll}\tau_{1} & \tau_{2}\end{array}\right]^{T}$

- $\tau_{1}, \tau_{2}$ are the control inputs as the two joints (torque inputs).

- $\theta_{1}, \theta_{2}, \dot{\theta_{1}}, \dot{\theta_{2}}$ are measured positions and angular velocities.

- $M(\theta)$ is the mass matrix.

- $C(\theta, \dot{\theta})$ is the Coriolis and centrifugal force matrix.

- $G(\theta)$ is the gravity matrix.

The mass, Coriolis and the gravity matrices are

$$
\begin{aligned}
M(\theta) & =\left[\begin{array}{cc}
P_{1}+P_{2}+2 P_{3} \cos \theta_{2} & P_{2}+P_{3} \cos \theta_{2} \\
P_{2}+P_{3} \cos \theta_{2} & P_{2}
\end{array}\right] \\
C(\theta, \dot{\theta}) & =\left[\begin{array}{cc}
b_{1}-P_{3} \dot{\theta_{2}} \sin \theta_{2} & -P_{3}\left(\dot{\theta_{1}}+\dot{\theta_{2}}\right) \sin \theta_{2} \\
-P_{3} \dot{\theta_{2}} \sin \theta_{2} & b_{2}
\end{array}\right] \\
G(\theta) & =\left[\begin{array}{c}
-g_{1} \sin \theta_{1}-g_{2} \sin \left(\theta_{1}+\theta_{2}\right) \\
-g_{2} \sin \left(\theta_{1}+\theta_{2}\right)
\end{array}\right]
\end{aligned}
$$

where 
- $P_{1}=m_{1} c_{1}^{2}+m_{2} l_{1}^{2}+I_{1}$

- $P_{2}=m_{2} c_{2}^{2}+I_{2}$

- $P_{3}=m_{2} l_{1} c_{2}$

- $g_{1}=\left(m_{1} c_{1}+m_{2} l_{1}\right) g$

- $g_{2}=m_{2} c_{2} g$

- $g$ is the acceleration due to gravity.

- $l_{1}$ and $l_{2}$ are the lengths of the first and the second link, respectively.

- $m_{1}$ and $m_{2}$ are the masses of the first and the second link, respectively.

- $I_{1}$ and $I_{2}$ are the inertias of the first and the second link, respectively.

- $c_{1}$ and $c_{2}$ are the centers of mass of the first and the second link, respectively.

- $b_{1}$ and $b_{2}$ are the damping in the first and the second joint, respectively.

Considering operation in the horizontal plane $(G(\theta)=0)$, we can represent the 2-DOF robot arm by a state space model in the following form.

$$
\dot{\boldsymbol{x}}=A(\theta, \dot{\theta}) \boldsymbol{x}+B(\theta) \tau
$$

where

$$
\begin{aligned}
& \boldsymbol{x}=\left[\begin{array}{llll}
\dot{\theta_{1}} & \dot{\theta_{2}} & \theta_{1} & \theta_{2}
\end{array}\right]^{T} \\
& A=\left[\begin{array}{cc}
-M^{-1} C & 0 \\
I & 0
\end{array}\right] \text { i.e, } \\
& A=\left[\begin{array}{llll}
A_{11} & A_{12} & A_{13} & A_{14} \\
A_{21} & A_{22} & A_{23} & A_{24} \\
A_{31} & A_{32} & A_{33} & A_{34} \\
A_{41} & A_{42} & A_{43} & A_{44}
\end{array}\right] \\
& A_{11}=\frac{P_{2}\left(b_{1}-P_{3} \dot{\theta}_{2} \sin \left(\theta_{2}\right)\right.}{P_{3}^{2} \cos ^{2}\left(\theta_{2}\right)-P_{1} P_{2}}-\frac{P_{3} \dot{\theta}_{2} \sin \left(\theta_{2}\right)\left(P_{2}+P_{3} \cos \left(\theta_{2}\right)\right)}{P_{3}^{2} \cos ^{2}\left(\theta_{2}\right)-P_{1} P_{2}} \\
& A_{12}=-\frac{b 2\left(P_{2}+P_{3} \cos \left(\theta_{2}\right)\right)}{P_{3}^{2} \cos ^{2}\left(\theta_{2}\right)-P_{1} P_{2}}-\frac{P_{2} P_{3} \sin \left(\theta_{2}\right)\left(\dot{\theta}_{1}+\dot{\theta}_{2}\right)}{P_{3}^{2} \cos ^{2}\left(\theta_{2}\right)-P_{1} P_{2}} \\
& A_{21}=\frac{P_{3} \dot{\theta}_{2} \sin \left(\theta_{2}\right)\left(P_{1}+P_{2}+2 P_{3} \cos \left(\theta_{2}\right)\right)}{P_{3}^{2} \cos ^{2}\left(\theta_{2}\right)-P_{1} P_{2}}-\frac{\left(P_{2}+P_{3} \cos \left(\theta_{2}\right)\right)\left(b_{1}-P_{3} \dot{\theta}_{2} \sin \left(\theta_{2}\right)\right)}{P_{3}^{2} \cos ^{2}\left(\theta_{2}\right)-P_{1} P_{2}} \\
& A_{22}=\frac{b 2\left(P_{1}+P_{2}+2 P_{3} \cos \left(\theta_{2}\right)\right)}{P_{3}^{2} \cos ^{2}\left(\theta_{2}\right)-P_{1} P_{2}}+\frac{P_{3} \sin \left(\theta_{2}\right)\left(P_{2}+P_{3} \cos \left(\theta_{2}\right)\right)\left(\dot{\theta}_{1}+\dot{\theta}_{2}\right)}{P_{3}^{2} \cos ^{2}\left(\theta_{2}\right)-P_{1} P_{2}} \\
& A_{13}=A_{14}=A_{23}=A_{24}=A_{32}=A_{33}=A_{34}=A_{41}=A_{43}=A_{44}=0 \\
& A_{31}=A_{42}=1
\end{aligned}
$$


$B=\left[\begin{array}{c}M^{-1} \\ \mathbf{0}\end{array}\right]$ i.e.,

$B=\left[\begin{array}{cc}-\frac{P_{2}}{P_{3}^{2} \cos ^{2}\left(\theta_{2}\right)-P_{1} P_{2}} & \frac{P_{2}+P_{3} \cos \left(\theta_{2}\right)}{P_{3}^{2} \cos ^{2}\left(\theta_{2}\right)-P_{1} P_{2}} \\ \frac{P_{2}+P_{3} \cos \left(\theta_{2}\right)}{P_{3}^{2} \cos ^{2}\left(\theta_{2}\right)-P_{1} P_{2}} & -\frac{P_{1}+P_{2}+2 P_{3} \cos \left(\theta_{2}\right)}{P_{3}^{2} \cos ^{2}\left(\theta_{2}\right)-P_{1} P_{2}}\end{array}\right]$

Examining the above nonlinear model indicates the presence of 6 nonlinear terms as follows.

$$
\begin{aligned}
& z_{1}=\frac{1}{\mathrm{P}_{3}{ }^{2} \cos \left(\theta_{2}\right)^{2}-\mathrm{P}_{1} \mathrm{P}_{2}} \\
& z_{2}=\frac{\cos \left(\theta_{2}\right)}{\mathrm{P}_{3}{ }^{2} \cos \left(\theta_{2}\right)^{2}-\mathrm{P}_{1} \mathrm{P}_{2}} \\
& z_{3}=\frac{\dot{\theta_{2}} \sin \left(\theta_{2}\right)}{\mathrm{P}_{3}{ }^{2} \cos \left(\theta_{2}\right)^{2}-\mathrm{P}_{1} \mathrm{P}_{2}} \\
& z_{4}=\frac{\dot{\theta_{2}} \cos \left(\theta_{2}\right) \sin \left(\theta_{2}\right)}{\mathrm{P}_{3}{ }^{2} \cos \left(\theta_{2}\right)^{2}-\mathrm{P}_{1} \mathrm{P}_{2}} \\
& z_{5}=\frac{\dot{\theta_{1}} \sin \left(\theta_{2}\right)}{\mathrm{P}_{3}{ }^{2} \cos \left(\theta_{2}\right)^{2}-\mathrm{P}_{1} \mathrm{P}_{2}} \\
& z_{6}=\frac{\dot{\theta_{1} \cos \left(\theta_{2}\right) \sin \left(\theta_{2}\right)}}{\mathrm{P}_{3}{ }^{2} \cos \left(\theta_{2}\right)^{2}-\mathrm{P}_{1} \mathrm{P}_{2}}
\end{aligned}
$$

The term $A_{11}$ for instance can be written in terms of the above nonlinearities as

$$
A_{11}=P_{2} b_{1} z_{1}-2 P_{3} P_{2} z_{3}-P_{3}^{2} z_{4}
$$

Similarly all other elements in the $A$ matrix can be written in terms of the above 6 nonlinearities.

The above 6 nonlinear terms result in $2^{6}$ rules. The rules will be of the form $R_{i}$ : If $z_{1}$ is $N_{i}^{1}$ and $z_{2}$ is $N_{i}^{2}$ and $z_{3}$ is $N_{i}^{3}$ and $z_{4}$ is $N_{i}^{4}$ and $z_{5}$ is $N_{i}^{5}$ and $z_{6}$ is $N_{i}^{6}$ then

$$
\begin{aligned}
\dot{\boldsymbol{x}} & =A_{i} \boldsymbol{x}+B_{i} \boldsymbol{u} \\
\boldsymbol{y} & =C_{i} \boldsymbol{x}
\end{aligned}
$$

where $i=1,2, \ldots, r$. The number of rules in this fuzzy model is 64 . Neglecting Coriolis and centrifugal forces, the fuzzy model can be made simpler and the number of rules can be reduced to 4. This case is described in Chapter 3. For presenting the results in this thesis, we used the simplified 2-DOF robot arm presented in Chapter 3. 


\section{Appendix C}

\section{Robust controller design with relaxed stability conditions}

This appendix presents the proof of Theorem 8 and Theorem 10. The content of these proofs form the basis of theorems $9,11,12$ and 13 .

The proof of the Theorem 8 is as follows. For the system given by

$$
\begin{aligned}
\dot{\boldsymbol{x}} & =\sum_{i=1}^{r} h_{i}(\boldsymbol{z})\left(A_{i} \boldsymbol{x}+B_{i} \boldsymbol{u}+M_{i} A_{\delta i} \boldsymbol{x}\right) \\
\boldsymbol{y} & =C \boldsymbol{x}
\end{aligned}
$$

the control input generated by the PDC

$$
\boldsymbol{u}=-\sum_{j=1}^{r} h_{j}(\boldsymbol{z}) F_{j} \boldsymbol{x}
$$

results in the following closed loop system dynamics

$$
\begin{aligned}
\dot{\boldsymbol{x}}= & \sum_{i=1}^{r} \sum_{j=1}^{r} h_{i}(\boldsymbol{z}) h_{j}(\boldsymbol{z})\left(A_{i}-B_{i} F_{j}+M_{i} A_{\delta i}\right) \boldsymbol{x} \\
= & \sum_{i=1}^{r} h_{i}{ }^{2}(\boldsymbol{z})\left(A_{i}-B_{i} F_{i}+M_{i} A_{\delta i}\right) \boldsymbol{x} \\
& +\sum_{i=1}^{r} \sum_{i<j} h_{i}(\boldsymbol{z}) h_{j}(\boldsymbol{z})\left(A_{i}-B_{i} F_{j}+A_{j}-B_{j} F_{i}+M_{i} A_{\delta i}+M_{j} A_{\delta j}\right) \boldsymbol{x}
\end{aligned}
$$

Considering a common quadratic Lyapunov function

$$
V=\boldsymbol{x}^{T} P \boldsymbol{x}
$$




$$
\begin{aligned}
& \dot{V}=\boldsymbol{x}^{T} P \dot{\boldsymbol{x}}+\dot{\boldsymbol{x}}^{T} P \boldsymbol{x} \\
& =\sum_{i=1}^{r} h_{i}{ }^{2}(\boldsymbol{z}) \boldsymbol{x}^{T}\left[P\left(A_{i}-B_{i} F_{i}+M_{i} A_{\delta i}\right)+\left(A_{i}-B_{i} F_{i}+M_{i} A_{\delta i}\right)^{T} P\right] \boldsymbol{x} \\
& +\sum_{i=1}^{r} \sum_{i<j} h_{i}(\boldsymbol{z}) h_{j}(\boldsymbol{z}) \boldsymbol{x}^{T}\left[P\left(A_{i}-B_{i} F_{j}+M_{i} A_{\delta i}\right)+\left(A_{i}-B_{i} F_{j}+M_{i} A_{\delta i}\right)^{T} P\right. \\
& \left.+P\left(A_{j}-B_{j} F_{i}+M_{j} A_{\delta j}\right)+\left(A_{j}-B_{j} F_{i}+M_{j} A_{\delta j}\right)^{T} P\right] \boldsymbol{x} \\
& =\sum_{i=1}^{r} h_{i}{ }^{2}(\boldsymbol{z}) \boldsymbol{x}^{T}\left[P\left(A_{i}-B_{i} F_{i}\right)+\left(A_{i}-B_{i} F_{i}\right)^{T} P+P\left(M_{i} A_{\delta i}\right)+\left(M_{i} A_{\delta i}\right)^{T} P\right] \boldsymbol{x} \\
& +\sum_{i=1}^{r} \sum_{i<j} h_{i}(\boldsymbol{z}) h_{j}(\boldsymbol{z}) \boldsymbol{x}^{T}\left[P\left(A_{i}-B_{i} F_{j}\right)+\left(A_{i}-B_{i} F_{j}\right)^{T} P+P\left(M_{i} A_{\delta i}\right)+\left(M_{i} A_{\delta i}\right)^{T} P\right. \\
& \left.+P\left(A_{j}-B_{j} F_{i}\right)+\left(A_{j}-B_{j} F_{i}\right)^{T} P+P\left(M_{j} A_{\delta j}\right)+\left(M_{j} A_{\delta j}\right)^{T} P\right] \boldsymbol{x} \\
& =\sum_{i=1}^{r} h_{i}{ }^{2}(\boldsymbol{z}) \boldsymbol{x}^{T}\left[P\left(A_{i}-B_{i} F_{i}\right)+\left(A_{i}-B_{i} F_{i}\right)^{T} P+P\left(M_{i} A_{\delta i}\right)+\left(M_{i} A_{\delta i}\right)^{T} P\right. \\
& \left.+P M_{i} M_{i}^{T} P-P M_{i} M_{i}^{T} P+A_{\delta i}^{T} A_{\delta i}-A_{\delta i}^{T} A_{\delta i}\right] \boldsymbol{x} \\
& +\sum_{i=1}^{r} \sum_{i<j} h_{i}(\boldsymbol{z}) h_{j}(\boldsymbol{z}) \boldsymbol{x}^{T}\left[P\left(A_{i}-B_{i} F_{j}\right)+\left(A_{i}-B_{i} F_{j}\right)^{T} P+P\left(M_{i} A_{\delta i}\right)+\left(M_{i} A_{\delta i}\right)^{T} P\right. \\
& +P\left(A_{j}-B_{j} F_{i}\right)+\left(A_{j}-B_{j} F_{i}\right)^{T} P+P\left(M_{j} A_{\delta j}\right)+\left(M_{j} A_{\delta j}\right)^{T} P+P M_{i} M_{i}^{T} P \\
& \left.-P M_{i} M_{i}^{T} P+A_{\delta i}^{T} A_{\delta i}-A_{\delta i}^{T} A_{\delta i}+P M_{j} M_{j}^{T} P-P M_{j} M_{j}^{T} P+A_{\delta j}^{T} A_{\delta j}-A_{\delta j}^{T} A_{\delta j}\right] \boldsymbol{x} \\
& =\sum_{i=1}^{r} h_{i}^{2}(\boldsymbol{z}) \boldsymbol{x}^{T}\left[P\left(A_{i}-B_{i} F_{i}\right)+\left(A_{i}-B_{i} F_{i}\right)^{T} P+P M_{i} M_{i}^{T} P+A_{\delta i}^{T} A_{\delta i}\right. \\
& \left.-P M_{i}\left(M_{i}^{T} P-A_{\delta i}\right)+A_{\delta i}^{T}\left(M_{i}^{T} P-A_{\delta i}\right)\right] \boldsymbol{x} \\
& +\sum_{i=1}^{r} \sum_{i<j} h_{i}(\boldsymbol{z}) h_{j}(\boldsymbol{z}) \boldsymbol{x}^{T}\left[P\left(A_{i}-B_{i} F_{j}\right)+\left(A_{i}-B_{i} F_{j}\right)^{T} P+P\left(A_{j}-B_{j} F_{i}\right)\right. \\
& +\left(A_{j}-B_{j} F_{i}\right)^{T} P+P M_{i} M_{i}^{T} P+P M_{j} M_{j}^{T} P+A_{\delta i}^{T} A_{\delta i}+A_{\delta j}^{T} A_{\delta j}-P M_{i}\left(M_{i}^{T} P-A_{\delta i}\right) \\
& \left.+A_{\delta i}^{T}\left(M_{i}^{T} P-A_{\delta i}\right)-P M_{j}\left(M_{j}^{T} P-A_{\delta j}\right)+A_{\delta j}^{T}\left(M_{j}^{T} P-A_{\delta j}\right)\right] \boldsymbol{x} \\
& =\sum_{i=1}^{r} h_{i}^{2}(\boldsymbol{z}) \boldsymbol{x}^{T}\left[P\left(A_{i}-B_{i} F_{i}\right)+\left(A_{i}-B_{i} F_{i}\right)^{T} P+P M_{i} M_{i}^{T} P+A_{\delta i}^{T} A_{\delta i}\right. \\
& \left.-\left(M_{i}^{T} P-A_{\delta i}\right)^{T}\left(M_{i}^{T} P-A_{\delta i}\right)\right] \boldsymbol{x} \\
& +\sum_{i=1}^{r} \sum_{i<j} h_{i}(\boldsymbol{z}) h_{j}(\boldsymbol{z}) \boldsymbol{x}^{T}\left[P\left(A_{i}-B_{i} F_{j}\right)+\left(A_{i}-B_{i} F_{j}\right)^{T} P+P\left(A_{j}-B_{j} F_{i}\right)\right. \\
& +\left(A_{j}-B_{j} F_{i}\right)^{T} P+P M_{i} M_{i}^{T} P+P M_{j} M_{j}^{T} P+A_{\delta i}^{T} A_{\delta i}+A_{\delta j}^{T} A_{\delta j} \\
& \left.-\left(M_{i}^{T} P-A_{\delta i}\right)^{T}\left(M_{i}^{T} P-A_{\delta i}\right)-\left(M_{j}^{T} P-A_{\delta j}\right)^{T}\left(M_{j}^{T} P-A_{\delta j}\right)\right] \boldsymbol{x}
\end{aligned}
$$


If

$$
\begin{aligned}
& P\left(A_{i}-B_{i} F_{j}\right)+\left(A_{i}-B_{i} F_{j}\right)^{T} P+P\left(A_{j}-B_{j} F_{i}\right)+\left(A_{j}-B_{j} F_{i}\right)^{T} P \\
& +P M_{i} M_{i}^{T} P+P M_{j} M_{j}^{T} P+\mu_{i}^{2} I+\mu_{j}^{2} I-2 Q_{0}<0
\end{aligned}
$$

for $i, j=1,2, \ldots, r$ and $i<j$ hold, then

$$
\begin{aligned}
\dot{V}(\boldsymbol{x})< & \sum_{i=1}^{r} h_{i}{ }^{2}(\boldsymbol{z}) \boldsymbol{x}^{T}\left[P\left(A_{i}-B_{i} F_{i}\right)+\left(A_{i}-B_{i} F_{i}\right)^{T} P+P M_{i} M_{i}^{T} P+A_{\delta i}^{T} A_{\delta i}\right. \\
& \left.-\left(M_{i}^{T} P-A_{\delta i}\right)^{T}\left(M_{i}^{T} P-A_{\delta i}\right)\right] \boldsymbol{x} \\
& +2 \sum_{i=1}^{r} \sum_{i<j} h_{i}(\boldsymbol{z}) h_{j}(\boldsymbol{z}) \boldsymbol{x}^{T} Q_{0} \boldsymbol{x}
\end{aligned}
$$

By Corollary 4 in [5], we have

$$
\begin{aligned}
\dot{V}(\boldsymbol{x})< & \sum_{i=1}^{r} h_{i}{ }^{2}(\boldsymbol{z}) \boldsymbol{x}^{T}\left[P\left(A_{i}-B_{i} F_{i}\right)+\left(A_{i}-B_{i} F_{i}\right)^{T} P+P M_{i} M_{i}^{T} P+A_{\delta i}^{T} A_{\delta i}\right. \\
& \left.-\left(M_{i}^{T} P-A_{\delta i}\right)^{T}\left(M_{i}^{T} P-A_{\delta i}\right)\right] \boldsymbol{x}+(s-1) \sum_{i=1}^{r} h_{i}{ }^{2}(\boldsymbol{z}) \boldsymbol{x}^{T} Q_{0} \boldsymbol{x}
\end{aligned}
$$

If

$$
P\left(A_{i}-B_{i} F_{i}\right)+\left(A_{i}-B_{i} F_{i}\right)^{T} P+P M_{i} M_{i}^{T} P+\mu_{i}^{2} I+(s-1) Q_{0}<0
$$

for $i, j=1,2, \ldots, r$ then $\dot{V}(\boldsymbol{x})<0$.

Left and right multiplying the inequalities in (C-5) and (C-6) by $P^{-1}$ and introducing a new variable $X=P^{-1}$, we can write

$$
\begin{aligned}
& \left(A_{i}-B_{i} F_{i}\right) X+X\left(A_{i}-B_{i} F_{i}\right)^{T}+M_{i} M_{i}^{T}+X \mu_{i}^{2} X+(s-1) X Q_{0} X<0 \\
& \left(A_{i}-B_{i} F_{j}\right) X+X\left(A_{i}-B_{i} F_{j}\right)^{T}+\left(A_{j}-B_{j} F_{i}\right) X+X\left(A_{j}-B_{j} F_{i}\right)^{T} \\
& +M_{i} M_{i}^{T}+M_{j} M_{j}^{T}+X \mu_{i}{ }^{2} X+X \mu_{j}{ }^{2} X+2 X Q_{0} X<0
\end{aligned}
$$

$i, j=1,2, \ldots, r$ and $i<j$.

With the change of variables $N_{i}=F_{i} X$ and hence $F_{i}=N_{i} P$, the inequalities in (4-16) become

$$
\begin{aligned}
& A_{i} X-B_{i} N_{i}+X A_{i}^{T}-N_{i}^{T} B_{i}+M_{i} M_{i}^{T}+X \mu_{i}^{2} X+(s-1) X Q_{0} X<0, \\
& \left.A_{i} X-B_{i} N_{j}+X A_{i}^{T}-N_{j}^{T} B_{i}\right)^{T}+A_{j} X-B_{j} N_{i}+X A_{j}^{T}-N_{i}^{T} B_{j} \\
& +M_{i} M_{i}^{T}+M_{j} M_{j}^{T}+X \mu_{i}^{2} X+X \mu_{j}^{2} X+2 X Q_{0} X<0
\end{aligned}
$$

$i, j=1,2, \ldots, r$ and $i<j$. 
Converting the conditions in (C-8) to linear matrix inequalities using Schur complements, we have

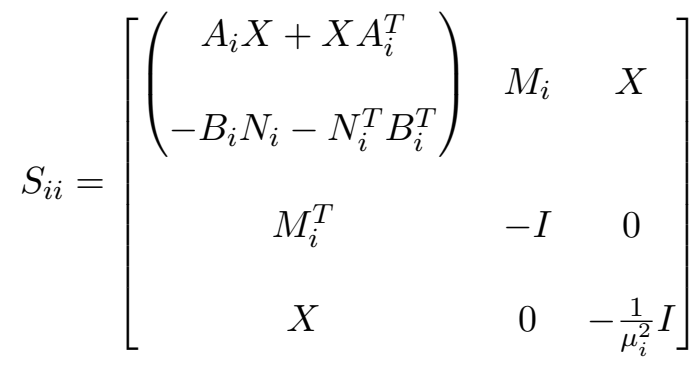

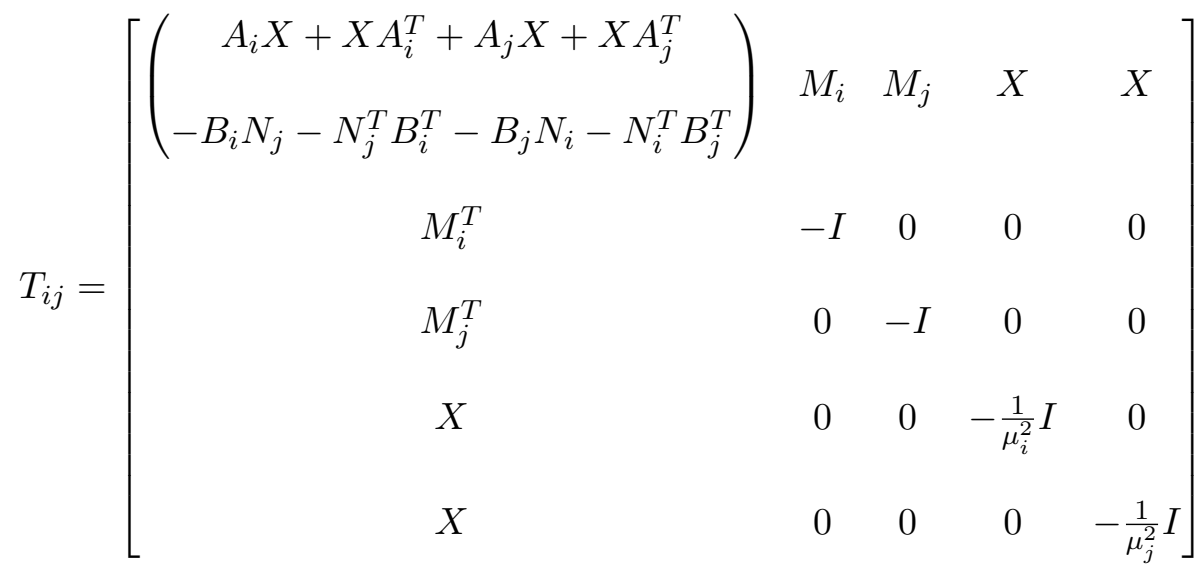

and

$$
\begin{array}{r}
S_{i i}+(s-1) Y_{0}<0 \\
T_{i j}+2 Y_{1}<0
\end{array}
$$

where

$$
\begin{aligned}
& Y_{1}=\operatorname{bkdiag}\left(\begin{array}{lll}
Y_{0} & 0 & 0
\end{array}\right), \\
& Y_{2}=\operatorname{bkdiag}\left(\begin{array}{lllll}
Y_{0} & 0 & 0 & 0 & 0
\end{array}\right), \\
& Y_{0}=X Q_{0} X
\end{aligned}
$$

Theorem 8 follows directly. This proof forms the basis of theorems 9,12 and 13 in Chapter 4 .

The following provides the basis for the proof of Theorem 10 which considers decay rate specification in the design of robust controller. For the uncertain Takagi-Sugeno (TS) system

$$
\begin{aligned}
\dot{\boldsymbol{x}} & =\sum_{i=1}^{r} h_{i}(\boldsymbol{z})\left(A_{i} \boldsymbol{x}+B_{i} \boldsymbol{u}+M_{i} A_{\delta i} \boldsymbol{x}\right) \\
\boldsymbol{y} & =C_{i} \boldsymbol{x}
\end{aligned}
$$

adapting Theorem 5 with $G_{i j}=\left(A_{i}-B_{i} F_{j}+M_{i} A_{\delta i}\right)$ and $Q=0$ and from Theorem 6 , we 
have equation (4-15) for the case with decay rate $\alpha$ as

$$
\begin{aligned}
& P\left(A_{i}-B_{i} F_{i}\right)+\left(A_{i}-B_{i} F_{i}\right)^{T} P+P M_{i} M_{i}^{T} P+\mu_{i}^{2} I+2 \alpha P<0 \\
& P\left(A_{i}-B_{i} F_{j}\right)+\left(A_{i}-B_{i} F_{j}\right)^{T} P+P\left(A_{j}-B_{j} F_{i}\right)+\left(A_{j}-B_{j} F_{i}\right)^{T} P \\
& +P M_{i} M_{i}^{T} P+P M_{j} M_{j}^{T} P+\mu_{i}^{2} I+\mu_{j}^{2} I+4 \alpha P<0
\end{aligned}
$$

hold for $i=1,2, \ldots, r$ and $i<j$, then we have $\dot{V}<-2 \alpha V$.

With $X=P^{-1}$ and a change of variable $N_{i}=F_{i} X$ we have the conditions

$$
\begin{aligned}
& A_{i} X-B_{i} N_{i}+X A_{i}^{T}-N_{i}^{T} B_{i}+M_{i} M_{i}^{T}+X \mu_{i}^{2} X+2 \alpha X<0 \\
& \left.A_{i} X-B_{i} N_{j}+X A_{i}^{T}-N_{j}^{T} B_{i}\right)^{T}+A_{j} X-B_{j} N_{i}+X A_{j}^{T}-N_{i}^{T} B_{j} \\
& +M_{i} M_{i}^{T}+M_{j} M_{j}^{T}+X \mu_{i}^{2} X+X \mu_{j}^{2} X+4 \alpha X<0
\end{aligned}
$$

$i=1,2, \ldots, r$ and $i<j$.

Theorem 10 is obtained by converting the above conditions to linear matrix inequality (LMI)s using Schur complements. 


\section{Computational complexity}

In this appendix, the computational complexity of the design in terms of the number of LMIs to be solved in designing the robust controller and the adaptive observer for the 2-DOF robot arm is studied.

\section{D-1 Number of LMIs in robust controller design}

We consider the number of LMIs that need to be solved when designing a robust controller using Theorem 11 along with the Theorem 14 from Chapter 4.

- The conditions $X>0$ from Theorem 11 and $X>\phi^{2} I$ from Theorem 14 can be combined into a single LMI. $X$ is of dimension $4 \times 4$.

- The number of LMIs introduced by conditions

$$
\begin{aligned}
& \hat{S}_{i i}<0, \\
& \hat{T}_{i j}<0, \quad i, j=1,2, \ldots, r \text { and } i<j \text { s.t } h_{i} h_{j} \neq 0
\end{aligned}
$$

from Theorem 11 is $\sum_{i=1}^{r} v_{m i}{ }^{1}$.

where $v_{m i}$ is a variable that takes values $i=1,2, \ldots, r$.

$\hat{S}_{i i}$ is of dimension $9 \times 9$ for the robot arm. In general, the dimension of $\hat{S}_{i i}$ is $\left(2 n_{x}+n_{d}\right) \times\left(2 n_{x}+n_{d}\right)$.

$\hat{T}_{i j}$ is of dimension $14 \times 14$ for the robot arm. In general, the dimension of $\hat{T}_{i j}$ is $\left(3 n_{x}+2 n_{d}\right) \times\left(3 n_{x}+2 n_{d}\right)$.

\footnotetext{
${ }^{1} \mathrm{mi}$ in the subscript stands for matrix inequalities
} 
- The LMI

$$
\left[\begin{array}{cc}
X & N_{i}^{T} \\
N_{i} & \zeta^{2} I
\end{array}\right] \geq 0
$$

from Theorem 14 adds further $r$ LMIs where $r$ is the number of rules in the TakagiSugeno (TS) model. Each of these LMIs is of dimension $6 \times 6$ for the robot arm. In general, the dimension of each of such LMI is $\left(n_{x}+n_{u}\right) \times\left(n_{x}+n_{u}\right)$.

Hence the total number of LMIs $n_{m i}$ is be given by

$$
n_{m i}=1+r+\sum_{i=1}^{r} v_{m i}
$$

Using equation D-4, the number of LMIs to be solved for the robot arm in 4 rule case is obtained as 15 and that in 64 rule case is 2145 . Thus the simplification from 64 rule to 4 rule model considerably reduces the number of LMIs to be solved and thus enables the solver to handle the system.

\section{D-2 Number of LMIs in adaptive observer design}

We consider the number of LMIs to be solved when designing the adaptive observer using Theorem 15 from Chapter 5 .

- The conditions $P>0$ and $Q>0$ are 2 LMIs. The matrices $P$ and $Q$ are both of dimension $4 \times 4$.

- The LMI

$$
\mathcal{H}\left(P\left(A_{i}-L_{i} C\right)\right)<-Q \quad i=1,2, \ldots, r
$$

results in $r$ additional LMIs each of dimension $4 \times 4$.

- The condition

$$
\left\|M_{i}\right\| \mu_{\max } \leq \frac{\lambda_{\min }(Q)}{\lambda_{\max }(P)}
$$

which is represented as the LMI

$$
\left[\begin{array}{cc}
Q-\mu_{\max }^{2} I & P \\
P & -I
\end{array}\right]
$$

of dimension $8 \times 8$ adds one to the LMI count.

Hence, for designing an adaptive observer for the 2-DOF robot arm using Theorem 15, the system of LMIs to be solved has the number of LMIs to be

$$
n_{m i}=r+3
$$

Using equation D-5, the number of LMIs to be solved in 4 rule case is obtained as 7 and that in 64 rule case is 67 . Hence, as the number of rules increase, the number of LMIs to be solved 
increases by the same number. For the design of robust controller and adaptive observer in this thesis, we have solved a total of 22 LMIs.

Multiple LMIs can be expressed as a single LMI [24]. In this case, the dimension of the resulting single matrix in the inequality is of importance. Combining multiple LMIs into a single one in case of robust controller gives a dimension of $\left(n_{x}+r\left(2 n_{x}+n_{d}\right)+\frac{r(r-1)}{2}\left(3 n_{x}+\right.\right.$ $\left.2 n_{d}\right)+r\left(n_{x}+n_{u}\right)$. Combining multiple LMIs into a single one in case adaptive observer results in a dimension of $\left(4 n_{x}+r n_{x}\right)$. Hence, combining multiple LMIs into a single LMI in case of robust controller and adaptive observer, the dimensions of the single LMI are:

- robust controller: $148 \times 148$

- adaptive observer: $34 \times 34$ 


\section{List of Acronyms}

$\begin{array}{ll}\text { TS } & \text { Takagi-Sugeno } \\ \text { LTI } & \text { linear time invariant } \\ \text { PDC } & \text { Parallel Distributed Compensator } \\ \text { LMI } & \text { linear matrix inequality }\end{array}$

\section{List of symbols and notations}

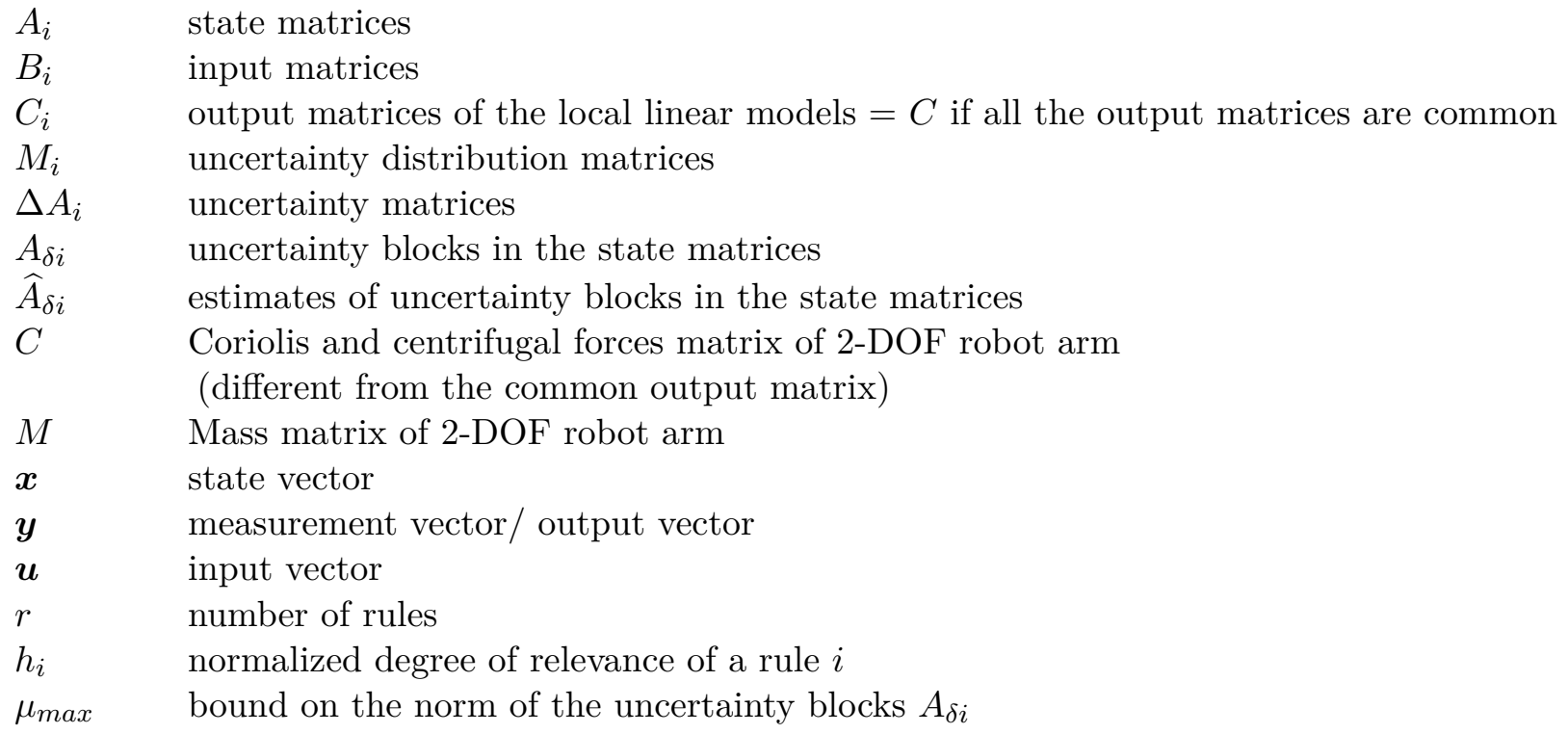




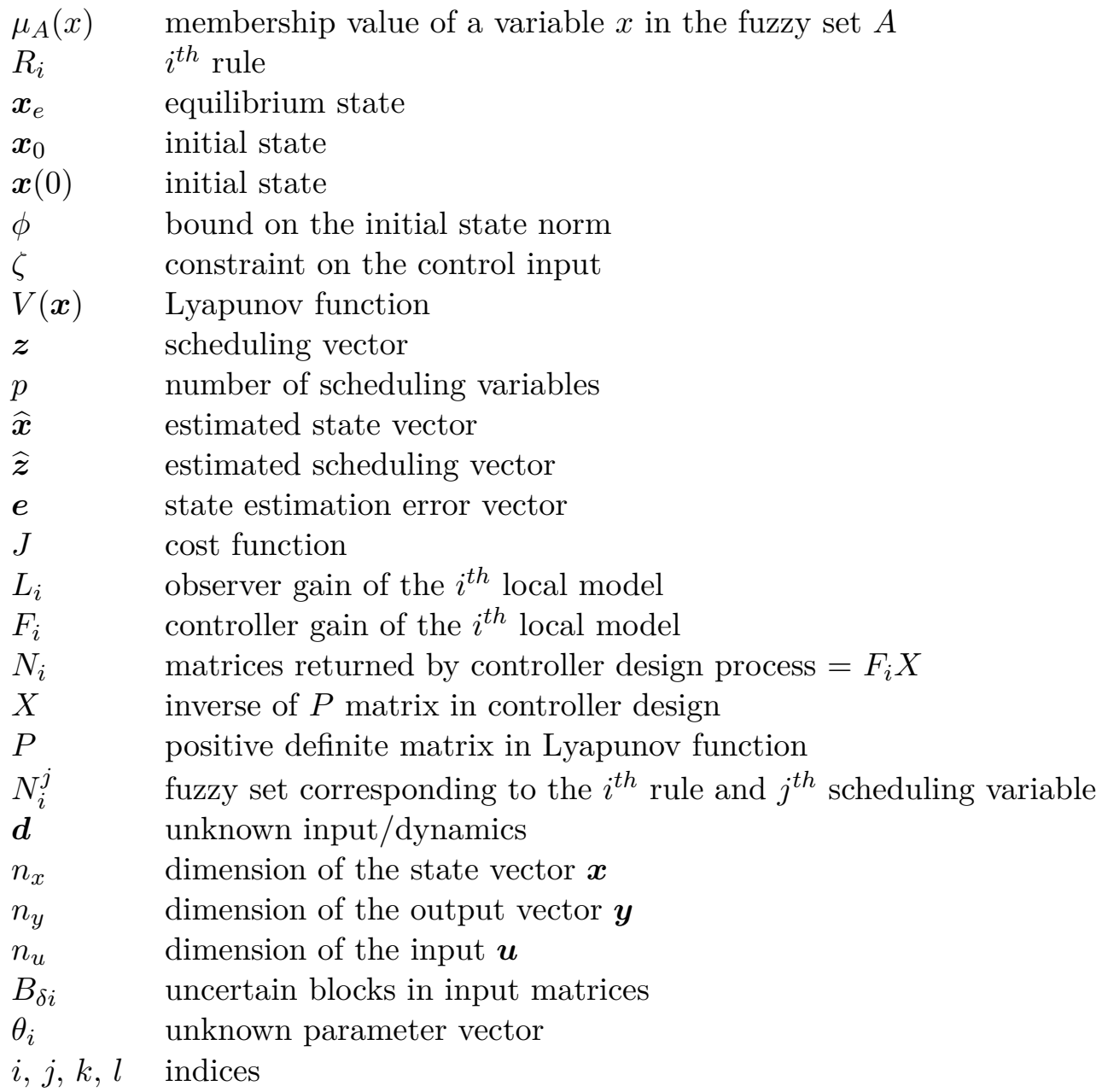




\section{References}

[1] Zs. Lendek, T. M. Guerra, R. Babuška, and B. De Schutter, Stability Analysis and Nonlinear Observer Design Using Takagi-Sugeno Fuzzy Models. Springer, 2010.

[2] H. K. Khalil, Nonlinear Systems. Prentice Hall, 1996.

[3] T. Takagi and M. Sugeno, "Fuzzy identification of systems and its applications to modeling and control," IEEE Transactions on Systems, Man, and Cybernetics., vol. 15, pp. 116-132, 1985.

[4] H. Ying, "General SISO Takagi-Sugeno fuzzy systems with linear rule consequent are universal approximators," IEEE Transactions on Fuzzy Systems, vol. 6, pp. 582-587, 1998.

[5] K. Tanaka and H. Wang, Fuzzy Control Systems Design and Analysis - A Linear Matrix Inequality Approach. John Wiley \& Sons, Inc, 2001.

[6] K. Tanaka and M. Sano, "On the concepts of regulator and observer of fuzzy control systems," in Proceedings of the IEEE World Congress on Computational Intelligence, (Orlando, FL , USA), pp. 767 - 772, June 1994.

[7] K. Tanaka and H. Wang, "Fuzzy regulators and fuzzy observers: a linear matrix inequality approach," in Proceedings of the 36th IEEE Conference on Decision and Control, (San Diego, CA, USA), pp. 1315-1320, December 1997.

[8] S. Zhen Bai and S. Tao Zhang, "Robust fuzzy control of uncertain nonlinear systems based on linear matrix inequalities," in Proceddings of the 7th World Congress on Intelligent Control and Automation, (Chongqing, China), pp. 4337 - 4341, June 2008.

[9] R. Kruse, J. E. Gebhardt, and F. Klowon, Foundations of Fuzzy Systems. New York, NY, USA: John Wiley \& Sons, Inc., 1994.

[10] C. C. Lee, "Fuzzy logic in control systems - fuzzy logic controller part I," IEEE Transactions on Systems, Man and Cybernetics, vol. 20, pp. 405-418, 1990. 
[11] R. Babuška, Knowledge-Based Control Systems. Delft University of Technology, 2010.

[12] M. Sugeno, "Fuzzy measures and fuzzy integrals - A survey," in Fuzzy Automata and Decision Processes, (Amsterdam, The Netherlands), pp. 89-102, 1977.

[13] M. Gupta and J. Qi, "Theory of T-norms and fuzzy inference methods," Fuzzy Sets and Systems, vol. 40, pp. 431-450, 1991.

[14] A. Kanehara and K.-Z. Liu, "On the capability of the TS model in describing nonlinear systems," Electrical Engineering in Japan (Original content in Denki Gakkai Ronbunshi in Japanese), pp. 376-382, 2009.

[15] K. Ogata, Modern Control Engineering. Prentice Hall India, 1996.

[16] K. Tanaka and M. Sano, "Fuzzy stability criterion of a class of nonlinear systems," Information Sciences, vol. 71, pp. 3-26, 1993.

[17] Y. Blanco, W. Perruquetti, and P. Borne, "Stability and stabilization of nonlinear systems and Takagi-Sugeno's fuzzy models," Mathematical Problems in Engineering, vol. 7, pp. 221-240, 2001.

[18] H. O. Wang, K. Tanaka, and M. F.Griffin, "An approach to fuzzy control of nonlinear systems: Stability and design issues," IEEE Transaction on Fuzzy Systems, vol. 4, pp. 14$23,1996$.

[19] K. Tanaka, T. Ikeda, and H. O. Wang, "Fuzzy regulators and fuzzy observers: Relaxed stability conditions and LMI-based designs," IEEE Transaction on Fuzzy Systems, vol. 2, pp. 250-265, 1998.

[20] X.-J. Ma, Z.-Q. Sun, and Y.-Y. He, "Analysis and design of fuzzy controller and fuzzy observer," IEEE Transactions on Fuzzy Systems, vol. 6, pp. 41-51, 1998.

[21] Zs. Lendek, Distributed Fuzzy and Stochastic Observers for Nonlinear Systems. PhD thesis, Technische Universiteit Delft, 2009.

[22] K. T. Hiroshi Ohtake and H. O. Wang, "Fuzzy modelling via sector nonlinearity concept," in Proceedings of Joint 9th IFSA World Congress and 20th NAFIPS International Conference, (Vancouver, Canada), pp. 127-132, 2001.

[23] L. Busoniu, B. D. Schutter, and R. Babuska, "Decentralized reinforcement learning control of a robotic manipulator," in Control, Automation, Robotics and Vision, 2006. ICARCV '06. 9th International Conference, 2006.

[24] S. Boyd, L. El Ghaoui, E. Feron, and V. Balakrishnan, Linear Matrix Inequalities in System and Control Theory, vol. 15 of Studies in Applied Mathematics. Philadelphia, PA: SIAM, June 1994. 